QUARTERLY OF APPLIED MATHEMATICS

VOLUME LXVIII, NUMBER 2

JUNE 2010, PAGES 293-331

S 0033-569X(10)01173-3

Article electronically published on February 18, 2010

\title{
LEFT-INVARIANT PARABOLIC EVOLUTIONS ON $S E(2)$ AND CONTOUR ENHANCEMENT VIA INVERTIBLE ORIENTATION SCORES \\ PART II: NONLINEAR LEFT-INVARIANT DIFFUSIONS ON INVERTIBLE ORIENTATION SCORES
}

\author{
$\mathrm{BY}$ \\ REMCO DUITS (Department of Mathematics/Computer Science and Department of Biomedical \\ Engineering, Eindhoven University of Technology, Den Dolech 2, P.O. Box 513, 5600MB Eindhoven, \\ The Netherlands) \\ AND \\ ERIK FRANKEN (Department of Biomedical Engineering, Eindhoven University of Technology, Den \\ Dolech 2, P.O. Box 513, 5600MB Eindhoven, The Netherlands)
}

\begin{abstract}
By means of a special type of wavelet unitary transform we construct an orientation score from a grey-value image. This orientation score is a complex-valued function on the 2D Euclidean motion group $S E(2)$ and gives us explicit information on the presence of local orientations in an image. As the transform between image and orientation score is unitary we can relate operators on images to operators on orientation scores in a robust manner. Here we consider nonlinear adaptive diffusion equations on these invertible orientation scores. These nonlinear diffusion equations lead to clear improvements of the celebrated standard "coherence enhancing diffusion" equations on images as they can enhance images with crossing contours. Here we employ differential geometry on $S E(2)$ to align the diffusion with optimized local coordinate systems attached to an orientation score, allowing us to include local features such as adaptive curvature in our diffusions.
\end{abstract}

1. Introduction. In many noisy medical images, elongated structures occur that cross each other. Therefore in the previous part, cf. [17, we proposed to enhance these elongated structures using linear diffusions on the Euclidean motion group $S E(2)$. In this

Received March 14, 2008.

2000 Mathematics Subject Classification. Primary 58J65, 49Q20, 22E30; Secondary 34B30, 37L05, 47D06.

Key words and phrases. Nonlinear diffusion equations on Lie groups, Cartan connection, fiber bundles, image analysis, coherence enhancing diffusion.

The Netherlands Organization for Scientific Research is gratefully acknowledged for financial support.

E-mail address: R.Duits@tue.nl

E-mail address: E.M.Franken@tue.nl 
part we go one step further and consider nonlinear diffusion on $S E(2)$. The advantage of the nonlinear approach is that we can adapt the diffusion process depending on the orientation confidence of local elongated structures. Just as in the previous part, we will process the image via diffusions on invertible orientation scores, which we will briefly explain next.

Image analysis usually starts with the sampling of a square integrable grey-value image

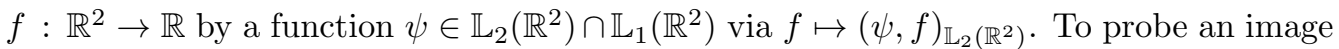
at every location $\mathbf{x} \in \mathbb{R}^{2}$ and in every direction $e^{i \theta} \in \mathbb{T}$ one translates and rotates an anisotropic wavelet $\psi$ by means of a representation $g \mapsto \mathcal{U}_{g}$ of the 2D Euclidean motion group $S E(2)$ given by

$$
\mathcal{U}_{g} \psi(\mathbf{y})=\psi\left(R_{\theta}^{-1}(\mathbf{y}-\mathbf{x})\right), g=\left(\mathbf{x}, e^{i \theta}\right) \in S E(2) .
$$

The result of such an image sampling is a function $\mathcal{W}_{\psi} f: S E(2) \rightarrow \mathbb{C}$ on the Euclidean motion group manifold $S E(2)=\mathbb{R}^{2} \rtimes \mathbb{T}$, which is given by

$$
\mathcal{W}_{\psi} f(g)=\left(\mathcal{U}_{g} \psi, f\right)_{\mathbb{L}_{2}\left(\mathbb{R}^{2}\right)}=\int_{\mathbb{R}^{2}} \overline{\psi\left(R_{\theta}^{-1}(\mathbf{y}-\mathbf{x})\right)} f(\mathbf{y}) \text { dy }, \text { with } g=\left(\mathbf{x}, e^{i \theta}\right),
$$

and where $R_{\theta}=\left(\begin{array}{cc}\cos \theta & -\sin \theta \\ \sin \theta & \cos \theta\end{array}\right) \in \mathrm{SO}(2)$. Throughout this article we refer to this function $\left(\mathbf{x}, e^{i \theta}\right) \mapsto \mathcal{W}_{\psi} f\left(\mathbf{x}, e^{i \theta}\right)$ as the orientation score $\mathcal{W}_{\psi} f$ of the grey-value image $f$.

As we have shown in [13, [11, ch:4.4, App.7.2] the transformation $\mathcal{W}_{\psi}$ is a unitary linear operator from $\mathbb{L}_{2}\left(\mathbb{R}^{2}\right)$ onto the unique reproducing kernel space $\mathbb{C}_{K}^{S E(2)}$ consisting of complex-valued functions on $S E(2)$ with reproducing kernel

$$
K(g, h)=\left(\mathcal{U}_{g} \psi, \mathcal{U}_{h} \psi\right)_{\mathbb{L}_{2}\left(\mathbb{R}^{2}\right)} .
$$

The generation of orientation scores and the reconstruction of images thereof has been the subject of previous publications, [11, 12, 14, 25], and in part I [17, we have derived the essential equality in the Fourier domain (of the spatial part only):

$$
\left\|\mathcal{W}_{\psi} f\right\|_{\mathbb{C}_{K}^{S E(2)}}^{2}=\int_{\mathbb{R}^{2}} \int_{\mathbb{T}}\left|\left(\mathcal{F} \mathcal{W}_{\psi} f\right)\left(\boldsymbol{\omega}, e^{i \theta}\right)\right|^{2} \mathrm{~d} \theta \frac{1}{M_{\psi}(\boldsymbol{\omega})} \mathrm{d} \boldsymbol{\omega}=\|f\|_{\mathbb{L}_{2}\left(\mathbb{R}^{2}\right)}^{2},
$$

where $M_{\psi}(\boldsymbol{\omega}):=\int_{0}^{2 \pi}\left|\mathcal{F} \psi\left(R_{\theta}^{T} \boldsymbol{\omega}\right)\right|^{2} \mathrm{~d} \theta$ and where we assume that $\psi$ is chosen such that $M_{\psi}>0$, so that $\frac{1}{M_{\psi}}$ is well-defined. Here $\mathcal{F}$ denotes the usual unitary Fourier transform on $\mathbb{L}_{2}\left(\mathbb{R}^{2}\right)$ given by $\mathcal{F} \psi(\boldsymbol{\omega})=\frac{1}{2 \pi} \int_{\mathbb{R}^{2}} \psi(\mathbf{x}) e^{-i \mathbf{x} \cdot \boldsymbol{\omega}} \mathrm{d} \mathbf{x}$.

From (1.4) we obtain the reconstruction formula

$$
f=\mathcal{W}_{\psi}^{*} \mathcal{W}_{\psi}[f]=\mathcal{F}^{-1}\left[\boldsymbol{\omega} \mapsto \int_{0}^{2 \pi} \mathcal{F}\left[\mathcal{W}_{\psi} f\left(\cdot, e^{i \theta}\right)\right](\boldsymbol{\omega}) \mathcal{F}\left[\mathcal{R}_{e^{i \theta}} \psi\right](\boldsymbol{\omega}) \mathrm{d} \theta M_{\psi}^{-1}(\boldsymbol{\omega})\right] .
$$

The transformation between images and orientation scores preserves the $\mathbb{L}_{2}$-norm iff

$$
\|f\|_{\mathbb{L}_{2}\left(\mathbb{R}^{2}\right)}^{2}=\left\|\mathcal{W}_{\psi} f\right\|_{\mathbb{L}_{2}(S E(2))}^{2} \Leftrightarrow M_{\psi}=1 .
$$

It can be shown that for kernels $\psi \in \mathbb{L}_{2}\left(\mathbb{R}^{2}\right) \cap \mathbb{L}_{1}\left(\mathbb{R}^{2}\right)$ the function $M_{\psi}$ is a continuous function vanishing at infinity. This means that for such kernels the wavelet transform $\mathcal{W}_{\psi}$ cannot be an isometry from $\mathbb{L}_{2}\left(\mathbb{R}^{2}\right)$ into $\mathbb{L}_{2}(S E(2))$.

This problem can be tackled by choosing $\psi$, within the dual space $\mathbb{H}^{-k, 2}\left(\mathbb{R}^{2}\right)$ of the $k$-th order, $k>1$, isotropic Sobolev space $\mathbb{H}^{k, 2}\left(\mathbb{R}^{2}\right)$ such that $M_{\psi}=1$. In this case the associated distributional transform $\mathfrak{W}_{\Psi}: \mathbb{H}^{k, 2}\left(\mathbb{R}^{2}\right) \rightarrow \mathbb{C}_{K}^{S E(2)}$, defined by $\mathfrak{W}_{\Psi} f\left(\mathbf{x}, e^{i \theta}\right)=$ $\left\langle\Psi, \mathcal{U}_{\left(\mathbf{x}, e^{i \theta}\right)^{-1}} f\right\rangle$, for all $f \in H^{k}\left(\mathbb{R}^{2}\right)$, extends to an isometry from $\mathbb{L}_{2}\left(\mathbb{R}^{2}\right)$ into $\mathbb{L}_{2}(S E(2))$. 


\begin{tabular}{|c|c|c|}
\hline $\begin{array}{c}\text { Image } \\
f \in \mathbb{L}_{2}\left(\mathbb{R}^{2}\right)\end{array}$ & $\stackrel{\mathcal{W}_{\psi}}{>}$ & $\begin{array}{c}\text { Orientation Score } \\
U_{f} \in \mathbb{C}_{K}^{S E(2)} \subset \mathbb{L}_{2}(S E(2))\end{array}$ \\
\hline$\Upsilon \psi$ & & $\bigvee_{\Phi}$ \\
\hline $\begin{array}{l}\text { Processed Image } \\
\Upsilon \Upsilon f]=\mathcal{W}_{\psi}^{*}\left[\Phi\left[U_{f}\right]\right]\end{array}$ & $\left(\mathcal{W}_{\psi}^{*}\right)^{\mathrm{ext}}=\mathcal{W}_{\psi}^{*} \mathbb{P}_{\psi}$ & $\begin{array}{c}\text { Processed Score } \\
\Phi\left[U_{f}\right] \in \mathbb{L}_{2}(S E(2))\end{array}$ \\
\hline
\end{tabular}

FIG. 1. Top Row: The complete scheme; for proper anisotropic wavelets $\psi$ the linear map $\mathcal{W}_{\psi}$ is unitary from $\mathbb{L}_{2}\left(\mathbb{R}^{2}\right)$ onto the closed subspace $\mathbb{C}_{K}^{S E(2)}$ of orientation scores. We can uniquely relate a transformation $\mathbb{P}_{\psi} \circ \Phi: \mathbb{C}_{K}^{S E(2)} \rightarrow \mathbb{C}_{K}^{S E(2)}$ on an orientation score to a transformation on an image $\Upsilon_{\psi}=\left(\mathcal{W}_{\psi}^{*}\right) \circ \mathbb{P}_{\psi} \circ \Phi \circ \mathcal{W}_{\psi}$, Here $\mathbb{P}_{\psi}$ denotes the orthogonal projection onto $\mathbb{C}_{K}^{S E(2)}$ given by $\mathbb{P}_{\psi} U(g)=\int_{S E(2)} K(g, h) U(h) \mathrm{d} h$, using the reproducing kernel (1.3).

Alternatively, one may restrict the transform $\mathcal{W}_{\psi}$ to the space of images $f \in \mathbb{L}_{2}^{\varrho}\left(\mathbb{R}^{2}\right)$, the so-called "disc-limited" images, whose Fourier transform has support within a given disc with radius $\varrho>0$ as motivated in Part I. If we now choose $\psi \in \mathbb{L}_{2}^{\varrho}\left(\mathbb{R}^{2}\right)$ such that $M_{\psi}=1_{B_{\mathbf{0}, \varrho}}$, where $B_{\mathbf{0}, \varrho}=\left\{\boldsymbol{\omega} \in \mathbb{R}^{2} \mid\|\boldsymbol{\omega}\|<\rho\right\}$, then the unitary operator $\mathcal{W}_{\psi}$ : $\mathbb{L}_{2}^{\varrho}\left(\mathbb{R}^{2}\right) \rightarrow \mathbb{C}_{K}^{S E(2)}$ between the space of disc-limited images and the space of orientation scores preserves the $\mathbb{L}_{2}$-norm.

These two approaches lead to two different classes of proper wavelets, as explained in [12, ch: 4.3 and ch: 4.4], 11. In both approaches the engineering rationale behind $M_{\psi}=1$ is that the auto-correlations of all rotated kernels $\mathcal{R}_{e^{i \theta}} \psi$ together fill up the Fourier-spectrum. Note that $M_{\psi}(\boldsymbol{\omega})=\mathcal{F}\left(\int_{0}^{2 \pi} \mathcal{R}_{e^{i \theta}} \psi * \mathcal{R}_{e^{i \theta}} \overline{\bar{\psi}} \mathrm{d} \theta\right)(\boldsymbol{\omega})$, with $\bar{\psi}(\mathbf{x})=\overline{\psi(-\mathbf{x})}$.

As a result for appropriate choice of $\psi$ (say $M_{\psi}=1$ ) a small perturbation on an image $f$ corresponds to a small perturbation on its orientation score $\mathcal{W}_{\psi} f$ and vice versa, and consequently operators $\Phi$ on the space of orientation scores are bijectively related to operators on images $\Upsilon_{\psi}$ in a stable manner by

$$
\Upsilon_{\psi} \leftrightarrow \Phi \Leftrightarrow \Upsilon_{\psi}=\mathcal{W}_{\psi}^{*} \circ \Phi \circ \mathcal{W}_{\psi}
$$

see Figure 1. This bijection is manifest if $\Phi$ maps the space of orientation scores $\mathbb{C}_{K}^{S E(2)}$ into itself. With the operators $\Phi$ we consider in this article this is usually not the case. Recall from part I [17] that this does not cause any problems if we naturally extend the adjoint, recall [17, eq. 4.4], to the space $\mathbb{L}_{2}(S E(2))$. However one should keep in mind that the effective operator from the space of orientation scores into itself is given by $\mathbb{P}_{\psi} \circ \Phi ;$ see Figure 1

Furthermore, in part I we have explained that $\Phi$ must be a nonlinear left-invariant operator. Therefore we considered collision distributions obtained from a forward linear diffusion resolvent and a backward linear diffusion resolvent; that is, in part I [17, eqs. 4.1 and 4.2] and [15], [13, [12, we considered the case where the operator $\Phi: \mathbb{C}_{K}^{S E(2)} \rightarrow$ $\mathbb{L}_{2}(S E(2))$ is given by $\left.\Phi\left(\mathcal{W}_{\psi} f\right)=\left(Q^{D, \mathbf{a}}(\underline{A})-\alpha I\right)^{-1}\left(\chi\left(\Re\left\{\mathcal{W}_{\psi} f\right\}\right)\right)\right) \cdot\left(\left(Q^{D, \mathbf{a}}(\underline{A})\right)^{*}-\right.$ $\alpha I)-1\left(\chi\left(\Re\left\{\mathcal{W}_{\psi} f\right\}\right)\right)$, where we recall the quadratic form $Q^{D, \mathbf{a}}(\underline{A})$ on the left-invariant 
vector fields on $S E(2)$ :

$$
\begin{aligned}
& \underline{\mathcal{A}}:=\left\{\mathcal{A}_{1}, \mathcal{A}_{2}, \mathcal{A}_{3}\right\}=\left\{\partial_{\theta}, \partial_{\xi}, \partial_{\eta}\right\}, \quad \xi=x \cos \theta+y \sin \theta, \eta=-x \sin \theta+y \cos \theta, \\
& Q^{\mathbf{D}, \mathbf{a}}(\underline{\mathcal{A}})=\sum_{i=1}^{3}\left(-a_{i} \mathcal{A}_{i}+\sum_{j=1}^{3} \mathcal{A}_{i} D_{i j} \mathcal{A}_{j}\right), a_{i}, D_{i j} \in \mathbb{R}, D^{T}=D \geq 0,
\end{aligned}
$$

with $\mathbf{a}=\left(a_{1}, a_{2}, a_{3}\right) \in \mathbb{R}^{3}, D=\left[D_{i j}\right] \in \mathbb{R}^{3 \times 3}$. Here we recall that $\chi$ was some monotonic grey-value transformation to put a soft threshold on weak responses in the orientation score. The linear parts of these operators, such as

$$
\begin{aligned}
\left(\left(\left(Q^{D, \mathbf{a}}(\underline{A})\right)^{*}-\alpha I\right)^{-1} U\right)(g) & =\left(\int_{0}^{\infty} K_{s}^{D,-\mathbf{a}} e^{-\alpha s} \mathrm{~d} s *_{S E(2)} U\right)(g) \\
& =\left(R_{\alpha}^{D,-\mathbf{a}} *_{S E(2)} U\right)(g),
\end{aligned}
$$

are easily computed by means of $S E(2)$-convolution with the corresponding Green's

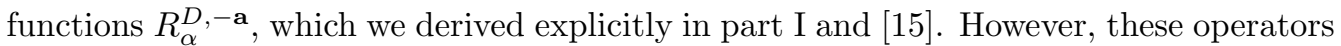

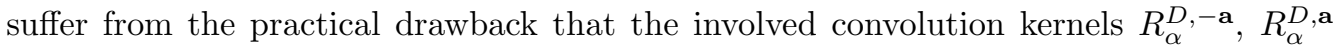
take care of constant diffusion and convection. In the approach presented in this article it is possible to incorporate curvature into contour completion and enhancement, as first reported (for contour completion) by August and Zucker [5].

In this part we go one step further: we propose $\Phi_{t}: \mathbb{C}_{K}^{S E(2)} \rightarrow \mathbb{L}_{2}(S E(2))$ as nonlinear adaptive diffusion equations on invertible orientation scores, with stopping time $t>0$. Here we will not consider products of linear resolvent equations, but we will consider nonlinear evolutions, without convection, given by

$$
\left\{\begin{array}{l}
\partial_{t} U(g, t)=Q^{D(U), \mathbf{a}=\mathbf{0}} U(g, t), \quad g \in S E(2), t>0, \\
U(g, 0)=\mathcal{W}_{\psi} f(g), \quad g \in S E(2),
\end{array}\right.
$$

where the conductivity $D(U)$ depends on the local differential structure of $(g, t) \mapsto$ $U(g, t)$.

Here we use locally optimal exponential curve fits to the absolute value of an orientation score. These optimal exponential curve fits provide a gauge frame attached to (the graph of) an orientation score at each position $g \in S E(2)$ in the 2D Euclidean motion group and will be used to locally align the diffusion on orientation scores. This locally adaptive alignment of diffusion is a common procedure in image processing, [30, [10], [39], but so far it has always been considered for diffusions directly on images.

The advantage of the more elaborate nonlinear, adaptive diffusions on invertible orientation scores is that the domain of orientation scores is the 2D Euclidean motion group $S E(2)$, which has a much richer structure than the domain of images, $\mathbb{R}^{2}$, allowing us to deal with (multiple) crossing curves in images.

1.1. Organization of Part II. Section 2 gives a quick review of locally adaptive diffusions in image processing. We finish this section with the so-called coherence-enhancing diffusions proposed in 39, where both the norm and the direction of the image gradient are used to steer the diffusion on the image. The basic idea is to diffuse tangent to edges/lines, not orthogonal to edges/lines, in images. The drawback of this approach is that at locations of crossing lines the direction of the gradient is ill-defined, resulting in ill-defined orientations and thereby artificial curvatures within the diffusion process. In 
the orientation score this typical crossing problem is automatically tackled as crossing line structures are torn apart by multiple convolutions with rotated versions of the oriented wavelet. Now in contrast to the differential structure in an image, the direction of each disentangled elongated structure in an orientation score is well-defined. Therefore, in Section 4 we consider coherence-enhancing diffusion on invertible orientation scores, meaning that we set $\Phi$ to be equal to a nonlinear left-invariant diffusion operator with a certain stopping time. Before we can provide these nonlinear diffusions we need some prerequisites from differential geometry on the Euclidean Motion group. This differential geometry will be explained in Section 3 and will be used to properly include orientation score adaptive features such as local curvature and deviation from horizontality in our nonlinear diffusion schemes.

Section 3 is organized as follows. In Subsection 3.1 we reformulate the coherenceenhancing diffusion schemes to stress the role of an invariant metric in an adaptive nonlinear diffusion scheme. Then in Subsection 3.2 we consider the design of an invariant metric on $S E(2)$, where by Theorem 3.1 we must choose between bi-invariance and nondegeneracy. Although bi-invariance is a common requirement in both the fields of mathematics (on symmetric Riemannian spaces, [24]) and computer vision [2], 3], 33], we show why we do not need it. As we explain in Lemma 3.3 and Corollary 3.4, operators $\Phi$ on orientation scores should be left-invariant and not right-invariant.

This brings us to a nondegenerate first fundamental form $\mathcal{G}_{\beta}$ (inducing a metric) on $S E(2)$, depending on a parameter $\beta$ with physical dimension $1 /[$ Length]. The induced metric does not coincide with the usual degenerate bi-invariant Cartan metric on $S E(2)$. Within this first fundamental form the parameter $\beta>0$ sets a balance between penalization of length and penalization of curvature of projections of curves to the spatial plane. As $\beta$ tends to zero this left-invariant inner product tends to the bi-invariant degenerate Cartan metric on $S E(2)$. For $\beta>0$ this first fundamental form is related to the nondegenerate Cartan metric on $S O(3)$ which can be embedded in $S E(2)$, as we will explain in Theorem 3.2. Furthermore in Subsection 3.2 we apply the Maurer-Cartan form and the thereby induced Cartan connection on $S E(2)$. This yields the covariant derivatives of vector fields on $S E(2)$, which we explain in Theorem 3.8. Then in Theorem 3.9 we show that our nonlinear diffusions on orientation scores can be expressed in these covariant derivatives and thereby the diffusions take place along the covariantly constant (i.e., auto-parallel) curves, which coincide with the exponential curves on $S E(2)$. The Cartan connection has constant curvature and torsion and so have the auto-parallel curves, which are indeed circular spirals.

Then in Subsection 3.3 we consider the definition and relevance of horizontal curves in $S E(2)$. To every $C^{1}$-curve $s \mapsto(x(s), y(s))$ one can associate a unique horizontal curve in $S E(2)$ by $s \mapsto\left(x(s), y(s), \theta(s)=\arg \left(x^{\prime}(s)+i y^{\prime}(s)\right)\right)$. This is relevant, since at regions $\Omega \subset S E(2)$ with strongly oriented responses $\left|\mathcal{W}_{\psi} f(g)\right|, g \in \Omega$, in the orientation score $g \mapsto$ $\mathcal{W}_{\psi} f(g)$ one would like to diffuse mainly along such horizontal curves. We will show that this requires a principal fiber bundle structure $P_{Y}$ on the domain of an orientation score, constructed from the unique subgroup $Y=\{(0, y, 0) \mid y \in \mathbb{R}\} \subset S E(2)$ with the property that constant right action on a horizontal curve again yields a horizontal curve. On this principal fiber bundle $P_{Y}=(S E(2), S E(2) / Y, \pi, R)$, with $Y=\{(0, y, 0) \mid y \in \mathbb{R}\}$ and 
$\pi(g)=g Y$ and $R_{h} g=g h$ we impose a Cartan-Ehresmann connection form. By definition, the kernel of this Cartan-Ehresmann connection form equals the horizontal part of each tangent space $T_{g}(S E(2))$ and coincides with the tangent space of all horizontal curves through $g$. This is explained in Theorem 3.13 , where we also equip $P_{Y}$ with the following left-invariant form $\mathrm{d} \theta \otimes \mathrm{d} \theta+\beta^{2}(\cos \theta \mathrm{d} x+\sin \theta \mathrm{d} y) \otimes(\cos \theta \mathrm{d} x+\sin \theta \mathrm{d} y)$, again parameterized by $\beta$, yielding a suitable left-invariant metric on $S E(2)$. This parameter is similar to the natural parameter in elastica curves, 29. Although there is a difference between the geodesics in $P_{Y}$ and elastica curves on $S E(2)$, we note that there is also a strong analogy between these curves; for more details, see [16. Here we will not make a detailed comparison between elastica curves in $\mathbb{R}^{2}$ and geodesics in $P_{Y}$. We will only derive the geodesics in $P_{Y}$ in Appendix A In contrast to well-known formulas for elastica curves, [29] our exact formula for the geodesics does not involve special functions.

In Subsection 3.4 we explain how one can obtain a best (horizontal) exponential curve fit to the orientation score data, locally at each $g \in S E(2)$. Later on in Subsection 4.1 we use the tangent vector of these optimal exponential curves to introduce a gaugecoordinate frame of left-invariant vector fields $\left\{\partial_{a}, \partial_{b}, \partial_{c}\right\}$, where $\partial_{b}$ is aligned with the best fitting (horizontal) exponential curve and $\partial_{a}$ and $\partial_{c}$ are tangent vectors orthogonal to $\partial_{b}$ with respect to the first fundamental form $\mathcal{G}_{\beta}$ of Section 3.2 .

Finally, in Section 4 we use the theoretical results of the previous section in our solution to a number of medical image analysis problems that require enhancement of (multiple) crossing elongated structures in noisy images. For details on the algorithmic side and medical image analysis applications, see our applied companion paper [20.

2. Previous work in the field of image analysis on locally adaptive diffusion. A scale space representation $u_{f}: \mathbb{R}^{d} \times \mathbb{R}^{+} \rightarrow \mathbb{R}$ of an image $f: \mathbb{R}^{d} \rightarrow \mathbb{R}$ is usually obtained by solving an evolution equation on the additive group $\left(\mathbb{R}^{d},+\right)$. The most common evolution equation, in image analysis, is the diffusion equation

$$
\left\{\begin{array}{l}
\partial_{s} u_{f}(\mathbf{x}, s)=\nabla_{\mathbf{x}} \cdot\left(C\left(u_{f}(\cdot, s)\right)(\mathbf{x}) \nabla_{\mathbf{x}} u_{f}\right)(\mathbf{x}, s) \\
u_{f}(\mathbf{x}, 0)=f(\mathbf{x})
\end{array}\right.
$$

where $C: \mathbb{L}_{2}\left(\mathbb{R}^{2}\right) \cap C^{2}\left(\mathbb{R}^{2}\right) \rightarrow C^{1}\left(\mathbb{R}^{2}\right)$ is a function which takes care of adaptive conductivity; that is, $C\left(u_{f}(\cdot, s)\right)(\mathbf{x})$ models the conductivity depending on the local differential structure at $\left(\mathbf{x}, s, u_{f}(\mathbf{x}, s)\right)$. If $C=1$ the solution is given by convolution $u_{f}(\mathbf{x}, s)=\left(G_{s} * f\right)(\mathbf{x})$ with a Gaussian kernel $G_{s}(\mathbf{x})=\frac{1}{(4 \pi s)^{\frac{d}{2}}} e^{-\frac{\|\mathbf{x}\|}{4 s}}$ with scale $s=\frac{1}{2} \sigma^{2}>0$.

As pointed out by Perona and Malik [31, nonlinear image adaptive isotropic diffusion is achieved by replacing $C=1$ by $C\left(u_{f}(\cdot, s)\right)(\mathbf{x})=c\left(\left\|\nabla_{\mathbf{x}} u_{f}(\mathbf{x}, s)\right\|\right)$, where $c: \mathbb{R}^{+} \rightarrow \mathbb{R}^{+}$ is some smooth strictly decaying positive function vanishing at infinity. This is based on the idea that if (locally) the gradient is large you do not want to diffuse too much. By restricting ourselves to positively valued $c>0$ one ensures that the diffusion is always forward, and thereby ill-posed backward diffusion is avoided. The common choices are

$$
c(t)=e^{-\frac{c}{\left(\frac{\lambda}{t}\right)^{2 p}}}, \quad c(t)=\frac{1}{\left(\frac{t}{\lambda}\right)^{2 p}+1} \text { and } c(t)=\frac{1}{\sqrt{\left(\frac{t}{\lambda}\right)^{2}+1}},
$$


involving parameters $p>\frac{1}{2}, \lambda>0$. The corresponding flux magnitude functions are given by $\phi(t)=t c(t)$, with $t=\left\|\nabla u_{f}\right\|$. Now the sign of

$$
\phi^{\prime}(t)=c(t)+t c^{\prime}(t)
$$

is important, since if $\phi^{\prime}(t)>0$, then the magnitude $\phi(t), t=\left\|\nabla u_{f}\right\|$, of the flux

$$
c\left(\left\|\nabla u_{f}\right\|\right) \nabla u_{f}
$$

(by the Gauss Theorem) increases as $\left\|\nabla u_{f}\right\|$ increases, whereas if $\phi^{\prime}(t)<0$ the magnitude $\phi\left(\left\|\nabla u_{f}\right\|\right)$ of the flux (2.3) decreases as $\left\|\nabla u_{f}\right\|$ increases. Typically, this introduces an extra "sharpening effect" of lines and edges. However, this sharpening effect should not be mistaken for ill-posed backward diffusion because, in all cases, $c(t) \geq 0$ for all $t>0$. To this end we note that the Perona and Malik equation can be rewritten in gauge coordinates $\{a, b\}$, with $a$ along the normalized gradient $\frac{1}{\left\|\nabla u_{f}\right\|} \nabla u_{f}$ and $b$ along the normalized vector $\frac{1}{\left\|\nabla u_{f}\right\|}\left(-\partial_{y} u_{f}, \partial_{x} u_{f}\right)$ orthogonal to the gradient, using (2.2):

$$
\begin{aligned}
\frac{\partial u_{f}}{\partial s} & =\operatorname{div}\left(c\left(\left\|\nabla u_{f}\right\|\right) \nabla u_{f}\right)=\frac{\partial}{\partial a}\left(c\left(\frac{\partial u_{f}}{\partial a}\right) \frac{\partial u_{f}}{\partial a}\right)+c\left(\frac{\partial u_{f}}{\partial a}\right) \frac{\partial^{2} u_{f}}{\partial b^{2}} \Leftrightarrow \\
\frac{\partial u_{f}}{\partial s} & =\phi^{\prime}\left(\frac{\partial u_{f}}{\partial a}\right) \frac{\partial^{2} u_{f}}{\partial a^{2}}+c\left(\frac{\partial u_{f}}{\partial a}\right) \frac{\partial^{2} u_{f}}{\partial b^{2}},
\end{aligned}
$$

with $\frac{\partial^{2} u_{f}}{\partial a^{2}}=\frac{1}{\left\|\nabla_{\mathbf{x}} u_{f}\right\|^{2}}\left(\nabla_{\mathbf{x}} u_{f}\right) H_{\mathbf{x}}\left[u_{f}\right]\left(\nabla_{\mathbf{x}} u_{f}\right)^{T}$ and $\frac{\partial u_{f}}{\partial a}=\left\|\nabla_{\mathbf{x}} u_{f}\right\|$.

A further improvement of the Perona and Malik scheme is introduced by Weickert [39], who also uses the direction of the gradient $\nabla_{\mathbf{x}} u_{f}$ of $u_{f}$, which is not used in the algorithms of Perona and Malik type. He proposed "coherence-enhancing diffusion" (CED), where the diffusion constant $c$ is replaced by a diffusion matrix:

$$
\begin{aligned}
& S\left(u_{f}(\cdot, s)\right)(\mathbf{x})=\left(G_{\sigma} * \nabla u_{f}(\cdot, s)\left(\nabla u_{f}(\cdot, s)\right)^{T}\right)(\mathbf{x}), \\
& C\left(u_{f}(\cdot, s)\right)(\mathbf{x})=\alpha I+\quad c \\
& (1-\alpha) e^{-\overline{\left(\lambda_{1}\left(S\left(u_{f}(\cdot, s)\right)(\mathbf{x})\right)-\lambda_{2}\left(S\left(u_{f}(\cdot, s)\right)(\mathbf{x})\right)\right)^{2}}} \mathbf{e}_{2}\left(S\left(u_{f}(\cdot, s)\right)(\mathbf{x})\right) \mathbf{e}_{2}^{T}\left(S\left(u_{f}(\cdot, s)\right)(\mathbf{x})\right),
\end{aligned}
$$

where $\alpha \in(0,1), c>0, \sigma>0$ are parameters and where the so-called "structure tensor" $S$, with eigenvalues $\left\{\lambda_{i}\left(S\left(u_{f}(\cdot, s)\right)(\mathbf{x})\right)\right\}_{i=1,2}$ is used to get a measure for local anisotropy $e^{-\frac{c}{\left(\lambda_{1}\left(S\left(u_{f}(\cdot, s)\right)(\mathbf{x})\right)-\lambda_{2}\left(S\left(u_{f}(\cdot, s)\right)(\mathbf{x})\right)\right)^{2}}}$ together with an orientation estimate $\mathbf{e}_{2}\left(S\left(u_{f}(\cdot, s)\right)(\mathbf{x})\right)$, which is the eigenvector of the structure tensor with smallest eigenvalue. In order to get robust orientation estimates it is essential to apply a componentwise smoothing on the so-called "structure-tensor field" $\nabla u_{f} \otimes \nabla u_{f}$. The amount of averaging of the structure tensor field is determined by $\sigma>0$. The CED method leads to useful and visually appealing diffusions of, for example, the famous Van Gogh paintings and fingerprint images; see Figure 2,

Nevertheless, this method fails in image analysis applications with crossing curves as it starts to create strong artificial curvatures at crossing locations where the direction of the gradient is ill-defined. As this is a major drawback in many imaging applications we are going to solve this problem by considering similar nonlinear adaptive evolution equations on invertible orientation scores. This coherence-enhancing diffusion via invertible orientation scores has two advantages over coherence-enhancing diffusion on images:

(1) In the domain $S E(2)=\mathbb{R}^{2} \rtimes \mathbb{T}$ of an (invertible) orientation score $\mathcal{W}_{\psi} f: S E(2) \rightarrow$ $\mathbb{R}$, crossing curves visible in an image $f: \mathbb{R}^{2} \rightarrow \mathbb{R}$ are torn apart by convolution with an oriented wavelet at multiple angles (1.2). Along the separated curves 


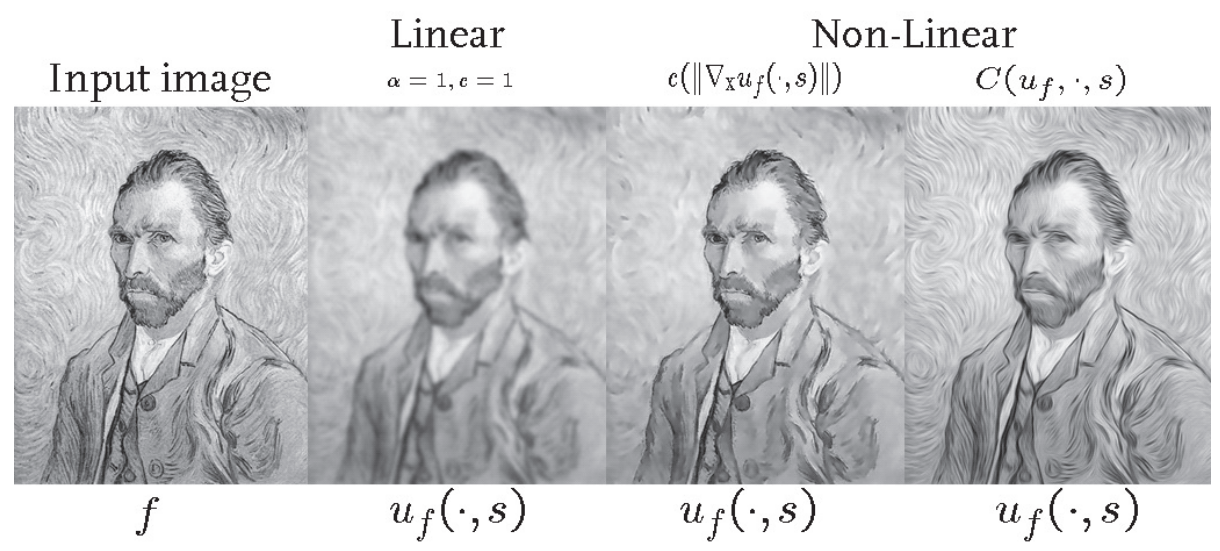

Fig. 2. From left to right: input image $f$ of the well-known portrait of Van Gogh, computed on comparable slices $u_{f}(\cdot, s)$ in a linear scale space representation $C=1$, Perona and Malik nonlinear scale space representation (left case in (2.1) and coherence-enhancing diffusion (CED) given by 2.5) in Weickert, 39.
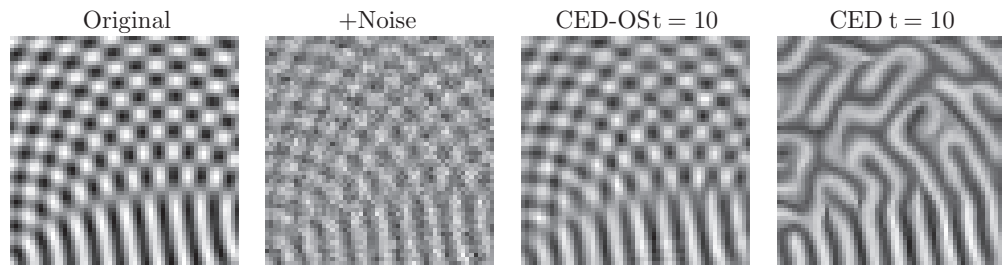

FIG. 3. Illustration of the typical different behavior of coherenceenhancing diffusion on images (CED) and coherence-enhancing diffusion via invertible orientation scores (CED-OS). Both methods are applied to the second image with noise and both evolutions are stopped at comparable stopping time $t=10$. Clearly, CED-OS preserves crossings of curves much better than CED (which creates artistic van Gogh-type patterns at crossings).

the direction of the gradient of an orientation score is well-defined. This allows us to diffuse coherently along the separate curves after which the inverse wavelet transformation will automatically merge the separate curves visible in a diffused orientation score $\Phi\left(\mathcal{W}_{\psi} f\right)$ into properly smoothed crossings visible in the final enhanced image $\Upsilon_{\psi}(f)$.

(2) In an orientation score $\left(x, y, e^{i \theta}\right) \mapsto \mathcal{W}_{\psi}(f)\left(x, y, e^{i \theta}\right)$ we have explicit information on local directions and we can easily measure curvature in a robust manner as we will explain in Subsection 3.4. This enables us to align the left-invariant diffusion $\mathcal{W}_{\psi} f \mapsto \Phi_{t}\left(\mathcal{W}_{\psi} f\right)$ on the orientation scores adaptively to the local differential structure in an evolving orientation score $\Phi_{t}\left(\mathcal{W}_{\psi} f\right)$.

See Figure 3 , 
Before we can translate these conceptual advantages into working image-processing algorithms, of which Fig. 3] shows an example result, we need to discuss some prerequisites from differential geometry, which will be the subject of the next section.

\section{Differential geometry on $S E(2)$.}

3.1. First fundamental forms underlying nonlinear diffusion schemes. In order to generalize the CED (coherence-enhancing diffusion) schemes to orientation scores we simply have to replace the left-invariant vector fields $\left\{\partial_{x}, \partial_{y}\right\}$ on the additive group $\left(\mathbb{R}^{2},+\right)$, by the left-invariant vector fields on $S E(2)$. To this end we formulate the standard coherence-enhancing diffusion equations on images (with conductivity (2.5)) as

$$
\left\{\begin{aligned}
\partial_{s} u_{f}(\mathbf{x}, s) & =\left(\nabla_{\mathbf{x}} \cdot \mathbf{S} \cdot\left(\begin{array}{cc}
\varepsilon & 0 \\
0 & (1-\varepsilon) e^{-\frac{c}{\lambda_{1}-\lambda_{2}}}+\varepsilon
\end{array}\right) \cdot \mathbf{S}^{-1} \cdot\left(\nabla_{\mathbf{x}} u_{f}(\cdot, s)\right)^{T}\right)(\mathbf{x}) \\
& =\left(\begin{array}{ll}
\partial_{a} & \partial_{b}
\end{array}\right)\left(\begin{array}{cc}
\varepsilon & 0 \\
0 & (1-\varepsilon) e^{-\frac{c}{\left(\lambda_{1}-\lambda_{2}\right)^{2}}}+\varepsilon
\end{array}\right)\left(\begin{array}{c}
\partial_{a} \\
\partial_{b}
\end{array}\right) u_{f}(\mathbf{x}, s), \quad \mathbf{x} \in \mathbb{R}^{2}, s>0, \\
u_{f}(\mathbf{x}, 0) & =f(\mathbf{x}), \quad \mathbf{x} \in \mathbb{R}^{2} .
\end{aligned}\right.
$$

Here we expressed the diffusion equations in both the global standard basis $\epsilon=$ $\left\{\mathbf{e}_{x}, \mathbf{e}_{y}\right\}:=\{(1,0),(0,1)\} \leftrightarrow\left\{\partial_{x}, \partial_{y}\right\}$ and in the locally adapted basis of eigenvectors of an auxiliary matrix $S\left(u_{f}(\cdot, s)\right)(\mathbf{x})$ (in image analysis known as the "structure tensor", recall (2.5) ):

$$
\alpha=\left\{\mathbf{e}_{1}, \mathbf{e}_{2}\right\}:=\left\{\mathbf{e}_{1}\left(S\left(u_{f}(\cdot, s)\right)(\mathbf{x})\right), \mathbf{e}_{2}\left(S\left(u_{f}(\cdot, s)\right)(\mathbf{x})\right)\right\} \leftrightarrow\left\{\partial_{a}, \partial_{b}\right\}
$$

with respective eigenvalues $\lambda_{k}:=\lambda_{k}\left(S\left(u_{f}(\cdot, s)\right)(\mathbf{x})\right), k=1,2$. The corresponding orthogonal basis transform which maps the standard basis vectors to the eigenvectors $\left\{\mathbf{e}_{1}, \mathbf{e}_{2}\right\}$ is denoted by $\mathbf{S}=\left(\mathbf{e}_{1} \mid \mathbf{e}_{2}\right)$ and we have $\left(\begin{array}{ll}\partial_{a} & \partial_{b}\end{array}\right)=\left(\begin{array}{ll}\partial_{x} & \partial_{y}\end{array}\right) \cdot \mathbf{S}$. At isotropic areas $\lambda_{1} \rightarrow \lambda_{2}$ and thereby the conductivity matrix becomes a multiple of the identity yielding isotropic diffusion only at isotropic areas, which is desirable for noise-removal.

In principle, one can consider linear diffusion equations by making the conductivity only adaptive to $f=u_{f}(\cdot, \cdot, 0)$. In such a case the mapping $f \mapsto u_{f}$ is still nonlinear, but the diffusion equation itself is linear. In a numerical finite difference scheme this means that the conductivity need not be updated as it is not dependent on time. Moreover, in this case the diffusion system will have a unique smooth solution, which is to our knowledge not a priori guaranteed in the case of nonlinear diffusions.

The diffusion/conductivity matrix in (3.1) is intuitively diagonalized along the local gauge-coordinate frame $\left\{\partial_{a}, \partial_{b}\right\}$ attached to the graph $\Gamma_{u_{f}(\cdot, \cdot, s)}=\left\{\left(x, y, u_{f}(x, y, s)\right) \mid\right.$ $\left.(x, y) \in \mathbb{R}^{2}\right\}$ of a blurred image $(x, y) \mapsto u_{f}(x, y, s), s \geq 0$. By equipping the space $\mathbb{R}^{2}$ with the usual metric where each tangent space $T_{\mathbf{x}}\left(\mathbb{R}^{2}\right)$ is identified with $T_{\mathbf{0}}\left(\mathbb{R}^{2}\right)$ by standard parallel transport on $\mathbb{R}^{2}$, the basis-transformation between the fixed coordinates and the gauge coordinates is unitary, so that $\mathbf{S}^{T}=\mathbf{S}^{-1}$ and thereby the diffusion is steered along a normalized, orthogonal, local gauge-coordinate frame $\left\{\partial_{a}, \partial_{b}\right\}$.

Instead of adapting the conductivity one can also intrinsically replace the standard first fundamental form on the image domain by a first fundamental form on the graph 
of an image in order to consider Laplace-Beltrami flow, as proposed by Sochen [33]:

$$
\left\{\begin{array}{l}
\partial_{s} u_{f}(x, y, s)=\frac{1}{\sqrt{\operatorname{det}\{G(x, y, s)\}}} \sum_{i=1}^{2} \sum_{j=1}^{2} \partial_{i}\left\{\sqrt{\operatorname{det}\{G(x, y, s)\}} G^{i j}(x, y, s) \partial_{j} u_{f}(\cdot, \cdot, s)\right\}(x, y), \\
\text { where we applied short notation } G(x, y, s):=C\left(u_{f}(\cdot, \cdot, s)\right)(x, y), \\
u_{f}(x, y, s=0)=f(x, y) \text { for all }(x, y) \in \mathbb{R}^{2} .
\end{array}\right.
$$

Now note that the right-hand side in the PDE in (3.1) can be rewritten as

$$
\sum_{i=1}^{2} \sum_{j=1}^{2} \partial_{i}\left\{G^{i j}(\cdot, \cdot, s) \partial_{j} u_{f}(\cdot, \cdot, s)\right\}(x, y),
$$

where $\left[G^{i j}(x, y, s)\right]_{i, j=1}^{2}$ denotes the inverse of the first fundamental form matrix $G(x, y, s)=\left[G_{i j}(x, y, s)\right]_{i, j=1}^{2}$. Here the inverse matrix $\left[G^{i j}(x, y, s)\right]_{i, j=1}^{2}$ equals the symmetric positive definite conductivity matrix $C\left(u_{f}(\cdot, \cdot, s)\right)(x, y)$, recall (2.5), evaluated at position $(x, y) \in \mathbb{R}^{2}$ at time $s>0$.

By the product rule for differentiation, the Laplace-Beltrami flow can be obtained by adding the following terms to the right-hand side of the PDE in (3.1):

$$
\frac{1}{\sqrt{\operatorname{det} G(x, y, s)}} \sum_{i=1}^{2} \partial_{i}\{\sqrt{\operatorname{det} G(\cdot, \cdot, s)}\}(x, y) \sum_{j=1}^{2} G^{i j}(x, y, s) \partial_{j} u_{f}(x, y, s) .
$$

For numerical reasons, however, we will not add these terms within this paper.

Now in order to generalize the coherence-enhancing diffusion schemes on images to coherence-enhancing diffusion schemes on orientation scores we must replace the leftinvariant vector fields on $\mathbb{R}^{2}$ by the left-invariant vector fields on $S E(2)$, as in (1.9), and in order to keep track of orthogonality and parallel transport in our diffusions we need an invariant first fundamental form $\mathcal{G}$ on $S E(2)$, rather than the trivial, bi-invariant, first fundamental form on $\left(\mathbb{R}^{2}, T\left(\mathbb{R}^{2}\right)\right)$, where each tangent space $T_{\mathbf{x}}\left(\mathbb{R}^{2}\right)$ is identified with $T_{\mathbf{0}}\left(\mathbb{R}^{2}\right)$ by standard parallel transport on $\mathbb{R}^{2}$, i.e. $\mathcal{G}_{\mathbb{R}^{2}}(\mathbf{x}, \mathbf{y})=\mathbf{x} \cdot \mathbf{y}=x^{1} y^{1}+x^{2} y^{2}$.

3.2. Design of the metric on $S E(2)$ : Bi-invariance versus nondegeneracy. In both the field of image analysis 2] and in mathematics (symmetric spaces) 24] it is very common to use bi-invariant metrics on groups. Here arises the first complication: as in $S E(2)$ no such bi-invariant, nondegenerate, first fundamental form (inducing a metric in the usual way) exists; see Theorem 3.1. Therefore we must choose between bi-invariance and nondegeneracy for the underlying metric in our nonlinear diffusion schemes. In this section we explain why we use a left-invariant, nondegenerate metric on $S E(2)$ as the underlying metric for our nonlinear diffusions on orientation scores. Furthermore, we will consider some differential geometry on $S E(2)$, which serves as an essential prerequisite for the full understanding and design of our diffusions on orientation scores later on 1

\footnotetext{
${ }^{1}$ It is not crucial to grasp all the details in Subsections $3.2[3.3$ to follow Sections 3.4 and 4 The reader who is not interested in the details can skip all proofs in Subsections $3.2 \quad 3.3$ and just consider Theorems 3.1 3.3 3.4 3.9 and Definition 3.11 and eqs. (3.5), (3.11), (3.25), (3.26), (3.31), (3.23), (3.24), (3.38).
} 
Theorem 3.1. The only real-valued left-invariant (symmetric, positive, semidefinite) first fundamental forms $\mathcal{G}: S E(2) \times T(S E(2)) \times T(S E(2)) \rightarrow \mathbb{C}$ on $S E(2)$ are given by

$$
\mathcal{G}=\sum_{i=1}^{3} \sum_{j=1}^{3} g_{i j} \mathrm{~d} \mathcal{A}^{i} \otimes \mathrm{d} \mathcal{A}^{j}, \quad g_{i j} \in \mathbb{R},
$$

where the dual basis $\left\{\mathrm{d} \mathcal{A}^{1}, \mathrm{~d} \mathcal{A}^{2}, \mathrm{~d} \mathcal{A}^{3}\right\} \subset(\mathcal{L}(S E(2)))^{*}$ of the dual space $(\mathcal{L}(S E(2)))^{*}$ of the vector space $\mathcal{L}(S E(2))$ of left-invariant vector fields spanned by

$$
\mathcal{A}_{1}=\partial_{\theta}, \mathcal{A}_{2}=\partial_{\xi}=\cos \theta \partial_{x}+\sin \theta \partial_{y}, \mathcal{A}_{3}=\partial_{\eta}=-\sin \theta \partial_{x}+\cos \theta \partial_{y},
$$

obtained by applying the derivative $\mathrm{d} \mathcal{R}$ of the right-regular representation $\mathcal{R}$ to the standard basis in the Lie algebra $\left\{A_{1}, A_{2}, A_{3}\right\}:=\left\{\partial_{x}, \partial_{y}, \partial_{\theta}\right\} \subset T_{e}(S E(2))$, is given by

$$
\mathrm{d} \mathcal{A}^{1}=\mathrm{d} \theta, \quad \mathrm{d} \mathcal{A}^{2}=\cos \theta \mathrm{d} x+\sin \theta \mathrm{d} y, \quad \mathrm{~d} \mathcal{A}^{3}=-\sin \theta \mathrm{d} x+\cos \theta \mathrm{d} y .
$$

The only (up to scalar multiplication) bi-invariant fundamental form on $S E(2)$ is degenerate and given by $\mathcal{G} \equiv \mathrm{d} \theta \otimes \mathrm{d} \theta$.

Proof. Recall from part I [17, ch:3] that $\mathrm{d} \mathcal{R}$ yields the fundamental isomorphism between the Lie algebras $T_{e}(S E(2))$ and $\mathcal{L}(S E(2))$, so $\mathcal{A}_{i}=\mathrm{d} \mathcal{R}\left(A_{i}\right)$ and $\left[\mathcal{A}_{i}, \mathcal{A}_{j}\right]=$ $\mathcal{A}_{i} \mathcal{A}_{j}-\mathcal{A}_{j} \mathcal{A}_{i}$. The dual basis (3.4) satisfies $\left\langle\mathrm{d} \mathcal{A}^{i}, \mathcal{A}_{j}\right\rangle=\delta_{j}^{i}$. Then by definition, $\mathcal{G}$ is

- left-invariant if $\forall_{h, g \in S E(2)} \forall_{X, Y \in \chi(S E(2))}: \mathcal{G}_{h}\left(X_{h}, Y_{h}\right)=\mathcal{G}_{g h}\left(\left(L_{g}\right)_{*} X_{h},\left(L_{g}\right)_{*} Y_{h}\right)$.

- right-invariant if $\forall_{h, g \in S E(2)} \forall_{X, Y \in \chi(S E(2))}: \mathcal{G}_{h}\left(X_{h}, Y_{h}\right)=\mathcal{G}_{h g}\left(\left(R_{g}\right)_{*} X_{h},\left(R_{g}\right)_{*} Y_{h}\right)$.

- inversion-invariant if $\forall_{h, g \in S E(2)} \forall_{X, Y \in \chi(S E(2))}$ : $\mathcal{G}_{r_{e}(g)}\left(\left(r_{e}\right)_{*} X_{g},\left(r_{e}\right)_{*} Y_{g}\right)=\mathcal{G}_{g}\left(X_{g}, Y_{g}\right)$.

- Ad-invariant if $\forall_{h, g \in S E(2)} \forall_{X, Y \in \chi(S E(2))}$ : $\mathcal{G}_{h g h^{-1}}\left(\operatorname{Ad}(h) X_{g}, \operatorname{Ad}(h) X_{g}\right)=\mathcal{G}_{g}\left(X_{g}, Y_{g}\right)$.

- reflection-invariant if $\forall_{h, g \in S E(2)} \forall_{X, Y \in \chi(S E(2))}$ : $\mathcal{G}_{r_{h}(g)}\left(\left(r_{h}\right)_{*} X_{g},\left(r_{h}\right)_{*} Y_{g}\right)=\mathcal{G}_{g}\left(X_{g}, Y_{g}\right)$.

Now the dual tangent space $\left(T_{g}(S E(2))\right)^{*}, g \in S E(2)$, is spanned by $\left\{\left.\mathrm{d} \mathcal{A}^{1}\right|_{g},\left.\mathrm{~d} \mathcal{A}^{2}\right|_{g}\right.$, $\left.\left.\mathrm{d} \mathcal{A}^{3}\right|_{g}\right\}$. As a result for all $g \in S E(2)$ there exist numbers $g_{i j}(g) \in \mathbb{R}, i, j=1,2,3$ such that

$$
\mathcal{G}_{g}=\left.\left.\sum_{i=1}^{3} \sum_{j=1}^{3} g_{i j}(g) \mathrm{d} \mathcal{A}^{i}\right|_{g} \otimes \mathrm{d} \mathcal{A}^{j}\right|_{g}
$$

Now $\mathcal{G}$ is left-invariant iff $\forall_{i, j \in\{1,2,3\}} \forall_{g \in S E(2)} \quad \mathcal{G}_{g}\left(\left.\mathcal{A}_{i}\right|_{g},\left.\mathcal{A}_{j}\right|_{g}\right)=\mathcal{G}_{e}\left(\left.\left(L_{g^{-1}}\right)^{*} \mathcal{A}_{i}\right|_{g}\right.$, $\left.\left.\left(L_{g^{-1}}\right)^{*} \mathcal{A}_{j}\right|_{g}\right)=\mathcal{G}_{e}\left(A_{i}, A_{j}\right)$, i.e. $\forall_{i, j \in\{1,2,3\}} \forall_{g \in S E(2)} g_{i j}(g)=g_{i j}(e)$. For the cases where $\mathcal{G}$ is bi-invariant we note that reflections around the unity element given by $r_{e}(g)=g^{-1}$ relate left multiplication $L_{g} h=g h$ to right multiplication $R_{g} h=h g$ since $R_{g}=r_{e} L_{g^{-1}} r_{e} \Leftrightarrow r_{e} R_{g^{-1}} r_{e}=L_{g}$ and reflections $h \mapsto r_{g}(h)=g h^{-1} g$ around element $g \in S E(2)$ follow by $r_{g}=L_{g} R_{g} r_{e}$ and the adjoint action is defined by $\operatorname{Ad}(g)=\left(R_{g^{-1}} L_{g}\right)_{*}$, so that, 35, Ch:V]:

$\mathcal{G}$ is both left and inversion-invariant $\Leftrightarrow \mathcal{G}$ is both left and right-invariant $\Leftrightarrow \mathcal{G}$ is both left and reflection-invariant $\Leftrightarrow \mathcal{G}$ is both left and Ad-invariant. 
This brings u 2 to the left-invariant Cartan form induced by the Killing-form $K$ (which is invariant under all Lie algebra automorphisms, [24, p.266], and in particular Ad):

$$
\begin{aligned}
\mathcal{G}_{g} & =-\left.\left.K\left(A_{i}, A_{j}\right) \mathrm{d} \mathcal{A}^{i}\right|_{g} \otimes \mathrm{d} \mathcal{A}^{j}\right|_{g}=\left.\left.\operatorname{trace}\left(\operatorname{ad}\left(\mathcal{A}_{i}\right) \circ \operatorname{ad}\left(\mathcal{A}_{j}\right)\right) \mathrm{d} \mathcal{A}^{i}\right|_{g} \otimes \mathrm{d} \mathcal{A}^{j}\right|_{g} \\
& =-\left.\left.\left\langle\mathrm{d} \mathcal{A}^{k}, \operatorname{ad}\left(\mathcal{A}_{i}\right) \circ \operatorname{ad}\left(\mathcal{A}_{j}\right) A_{k}\right\rangle \mathrm{d} \mathcal{A}^{i}\right|_{g} \otimes \mathrm{d} \mathcal{A}^{j}\right|_{g}=-\left.\left.c_{k j}^{l} c_{l i}^{k} \mathrm{~d} \mathcal{A}^{i}\right|_{g} \otimes \mathrm{d} \mathcal{A}^{j}\right|_{g},
\end{aligned}
$$

where $c_{i j}^{k}$ are the structure constants of the Lie algebra and $\operatorname{ad}\left(\mathcal{A}_{j}\right) \mathcal{A}_{i}=\left[\mathcal{A}_{i}, \mathcal{A}_{j}\right]=c_{i j}^{k} \mathcal{A}_{k}$. A direct computation of this Killing form yields $\mathcal{G}=\mathrm{d} \theta \otimes \mathrm{d} \theta$. It is not difficult to see that the metric given in (3.5) is the only both left- and Ad-invariant metric, since by left-invariance it can be written as (3.2) and by $\operatorname{Ad}_{g=\left(x, y, e^{i \theta}\right)}\left(\partial_{\theta}\right)=\partial_{\theta}-y \partial_{x}+x \partial_{y}$, $\operatorname{Ad}_{\left(x, y, e^{i \theta}\right)}\left(\partial_{x}\right)=\partial_{x}, \operatorname{Ad}_{\left(x, y, e^{i \theta}\right)}\left(\partial_{y}\right)=\partial_{y}$, the adjoint orbits are planes with fixed $\partial_{\theta}$ component on which the quadratic form is constant, so $g_{i j}=0$ if $(i, j) \neq(1,1)$.

Summarizing, we must choose between bi-invariance and invertibility. On the one hand, in Lie group theory it is common to maintain bi-invariance, and therefore we embed $S E(2)$ into $S O(3)$, on which the bi-invariant metric is nondegenerate. In Theorem 3.2 we present a parameterized class of compact groups $\left\{(S E(2))^{\beta} \mid 0<\beta \leq 1\right\}$, with $\lim _{\beta \downarrow 0}(S E(2))^{\beta}=S E(2)$ and $(2 \pi \mathbb{Z} \times\{0\} \times\{0\}) \backslash(S E(2))^{\beta=1} \equiv S O(3)$. Each member of this class admits a bi-invariant metric which is nondegenerate iff $\beta>0$. We use this class to derive covariant derivatives and Riemannian curvature on $(S E(2))^{\beta}$ and by taking the limit $\beta \downarrow 0$ we obtain covariant derivatives and sectional curvatures on $S E(2)$. On the other hand, by Lemma 3.3 and Corollary 3.4. operators on orientation scores should be left-invariant, not right-invariant, so we do not need right-invariance.

ThEOREM 3.2. The Euclidean motion group $S E(2)$ can be obtained by contraction from the group $S O(3)$, by means of the group $3^{3}(S E(2))^{\beta}, \beta \in[0,1]$, that arise by equipping the set $\mathbb{R}^{2} \times S_{1}$ with the group product

$$
\begin{aligned}
(x, y, \theta) \cdot \beta\left(x^{\prime}, y^{\prime}, \theta^{\prime}\right)= & \left(x+x^{\prime} \cos \theta \sqrt{1+\beta^{2} y^{2}}-y^{\prime} \sin \theta \sqrt{1+\beta^{2} y^{2}}, y+x^{\prime}\left(1+\beta^{2} y^{2}\right) \sin \theta\right. \\
& \left.+y^{\prime}\left(1+\beta^{2} y^{2}\right) \cos \theta, \theta+\theta^{\prime}-\beta^{2} x^{\prime} y \cos \theta+\beta^{2} y^{\prime} y \sin \theta \bmod 2 \pi\right) .
\end{aligned}
$$

One has $S E(2)=\lim _{\beta \downarrow 0}(S E(2))^{\beta}$ and $4 S(3) \equiv 2 \pi \mathbb{Z} \times\{0\} \times\{0\} \backslash(S E(2))^{\beta=1}$. The latter isomorphism (for a geometrical explanation, see Figure 4) is given by

$$
\begin{aligned}
& S O(3) \ni R_{\mathbf{e}_{z}, \tilde{\gamma}} R_{\mathbf{e}_{y}, \tilde{\beta}} R_{\mathbf{e}_{z}, \tilde{\alpha}} \leftrightarrow(x, y, \theta) \in(S E(2))^{\beta=1} \\
& \Leftrightarrow \tilde{\alpha}=x \text { and } \tilde{\beta}=\frac{\pi}{2}-\arctan (y) \text { and } \tilde{\gamma}=\theta,
\end{aligned}
$$

where the well-known Euler angle parametrization of $S O(3)$ is given by $R_{\mathbf{e}_{z}, \tilde{\gamma}} R_{\mathbf{e}_{y}, \tilde{\beta}} R_{\mathbf{e}_{z}, \tilde{\alpha}}$. The left-invariant vector fields on $(S E(2))^{\beta}$ are given by

$$
\begin{aligned}
& \mathcal{A}_{1}^{\beta}=\partial_{\theta}, \\
& \mathcal{A}_{2}^{\beta}=-\beta^{2} y \cos \theta \partial_{\theta}+\cos \theta \sqrt{1+\beta^{2} y^{2}} \partial_{x}+\sin \theta\left(1+\beta^{2} y^{2}\right) \partial_{y}, \\
& \mathcal{A}_{3}^{\beta}=\beta^{2} y \sin \theta \partial_{\theta}-\sin \theta \sqrt{1+\beta^{2} y^{2}} \partial_{x}+\cos \theta\left(1+\beta^{2} y^{2}\right) \partial_{y} .
\end{aligned}
$$

\footnotetext{
${ }^{2}$ Occasionally within this paper, as in 3.5 , we use the Einstein summation convention; i.e., we apply automatic summation over indices which appear both as upper and lower indices.

${ }^{3}$ The groups $(S E(2))^{\beta}, \beta \in[0,1]$ in this part II, connecting $S E(2)$ and $S O(3)$, should not be mistaken with the groups $(S E(2))_{t}, t \in[0,1]$, connecting $S E(2)$ and $H(3)$, used in part I [17.

${ }^{4}$ The quotient is taken only to ensure that the first variable $x$ of the group $(S E(2))^{\beta=1}$ is $2 \pi$-periodic.
} 
These vector fields form a 3D Lie algebra: $\left[\mathcal{A}_{1}^{\beta}, \mathcal{A}_{2}^{\beta}\right]=\mathcal{A}_{3}^{\beta}, \quad\left[\mathcal{A}_{1}^{\beta}, \mathcal{A}_{3}^{\beta}\right]=-\mathcal{A}_{2}^{\beta}$, $\left[\mathcal{A}_{2}^{\beta}, \mathcal{A}_{3}^{\beta}\right]=\beta^{2} \mathcal{A}_{1}^{\beta}$, which tends to $\mathcal{L}(S E(2))=\left\{\partial_{\theta}, \partial_{\xi}, \partial_{\eta}\right\}$ for $\beta \rightarrow 0$. Let the leftinvariant co-vectors $\left\{\mathrm{d} \mathcal{A}_{\beta}^{i}\right\}_{i=1}^{3}$ be given by $\left\langle\mathrm{d} \mathcal{A}_{\beta}^{i}, \mathcal{A}_{j}^{\beta}\right\rangle=\delta_{j}^{i}$. Then the bi-invariant first fundamental form $\mathcal{G}^{\beta}$ on $(S E(3))^{\beta}$ induced by the Killing form (as in (3.5) ) is given by

$$
\mathcal{G}^{\beta}=g_{i j} \mathrm{~d} \mathcal{A}_{\beta}^{i} \otimes \mathrm{d} \mathcal{A}_{\beta}^{j}, \text { with } G=\left[g_{i j}\right]=\left(\begin{array}{ccc}
1 & 0 & 0 \\
0 & \beta^{2} & 0 \\
0 & 0 & \beta^{2}
\end{array}\right) .
$$

As a result the groups $(S E(2))^{\beta}$ are compact symmetrif 5 Riemannian spaces iff $\beta>0$. The Riemann curvature tensor on these symmetric Riemannian spaces equals:

$$
\begin{array}{r}
R=\beta^{2} \mathcal{A}_{1}^{\beta} \otimes \mathrm{d} \mathcal{A}_{\beta}^{2} \otimes \mathrm{d} \mathcal{A}_{\beta}^{1} \wedge \mathrm{d} \mathcal{A}_{\beta}^{2}-\beta^{2} \mathcal{A}_{1}^{\beta} \otimes \mathrm{d} \mathcal{A}_{\beta}^{3} \otimes \mathrm{d} \mathcal{A}_{\beta}^{1} \wedge \mathrm{d} \mathcal{A}_{\beta}^{3} \\
+\beta^{2} \mathcal{A}_{2}^{\beta} \otimes \mathrm{d} \mathcal{A}_{\beta}^{3} \otimes \mathrm{d} \mathcal{A}_{\beta}^{2} \wedge \mathrm{d} \mathcal{A}_{\beta}^{3}-\beta^{2} \mathcal{A}_{3}^{\beta} \otimes \mathrm{d} \mathcal{A}_{\beta}^{2} \otimes \mathrm{d} \mathcal{A}_{\beta}^{3} \wedge \mathrm{d} \mathcal{A}_{\beta}^{2} \\
+\mathcal{A}_{2}^{\beta} \otimes \mathrm{d} \mathcal{A}_{\beta}^{1} \otimes \mathrm{d} \mathcal{A}_{\beta}^{1} \wedge \mathrm{d} \mathcal{A}_{\beta}^{2}+\mathcal{A}_{3}^{\beta} \otimes \mathrm{d} \mathcal{A}_{\beta}^{1} \otimes \mathrm{d} \mathcal{A}_{\beta}^{1} \wedge \mathrm{d} \mathcal{A}_{\beta}^{3} .
\end{array}
$$

Proof. The tangent space at the unity element $e=(0,0,0)$ of all groups $\left\{(S E(2))_{\beta}\right\}$ is the same for all $\beta \geq 0$ and it is spanned by $\left\{A_{1}, A_{2}, A_{3}\right\}=\left\{\partial_{x}, \partial_{y}, \partial_{\theta}\right\}$. Now the formula for the left-invariant invariant vector field $\mathcal{A}_{i}^{\beta}$ (3.7) directly follows by applying the derivative $\mathrm{d} \mathcal{R}$ of the right-regular representation $\mathcal{R}$ given by $\mathcal{R}_{g} \phi(h)=\phi(h g)$, as in (B.2), to $A_{i}, i=1,2,3$. Now with respect to the isomorphism (3.6) we note that the Euler angle parametrization of $S O(3)$ is given by $R_{\mathbf{e}_{z}, \tilde{\gamma}} R_{\mathbf{e}_{y}, \tilde{\beta}} R_{\mathbf{e}_{z}, \tilde{\alpha}}$. A basis of leftinvariant vector fields on $S O(3)$ (in Euler angles) is given by

$$
\mathcal{B}_{1}=\cot \tilde{\beta} \cos \tilde{\gamma} \partial_{\tilde{\gamma}}-\frac{\cos \tilde{\gamma}}{\sin \tilde{\beta}} \partial_{\tilde{\alpha}}+\sin \tilde{\gamma} \partial_{\tilde{\beta}}, \quad \mathcal{B}_{2}=-\cot \tilde{\beta} \sin \tilde{\gamma} \partial_{\tilde{\gamma}}-\frac{\cos \tilde{\gamma}}{\sin \tilde{\beta}} \partial_{\tilde{\alpha}}+\sin \tilde{\gamma} \partial_{\tilde{\beta}},
$$

and $\mathcal{B}_{3}=\partial_{\tilde{\gamma}}$, with commutators $\left[\mathcal{B}_{1}, \mathcal{B}_{2}\right]=\mathcal{B}_{3},\left[\mathcal{B}_{2}, \mathcal{B}_{3}\right]=\mathcal{B}_{1},\left[\mathcal{B}_{3}, \mathcal{B}_{1}\right]=\mathcal{B}_{2}$, [8, ch:9.10]. If we apply the coordinate transformation $\tilde{\alpha}=\beta x, \tilde{\beta}=\frac{\pi}{2}-\arctan (\beta y), \tilde{\gamma}=\theta$ and multiply $\mathcal{B}_{1}$ and $\mathcal{B}_{2}$ by $\beta$ we obtain the left-invariant vector fields (3.7). Therefore these vector fields $\left\{\mathcal{A}_{1}^{\beta}, \mathcal{A}_{2}^{\beta}, \mathcal{A}_{3}^{\beta}\right\}$ again form a three-dimensional Lie algebra:

$$
\left[\mathcal{A}_{1}^{\beta}, \mathcal{A}_{2}^{\beta}\right]=\mathcal{A}_{3}^{\beta}, \quad\left[\mathcal{A}_{1}^{\beta}, \mathcal{A}_{3}^{\beta}\right]=-\mathcal{A}_{2}^{\beta}, \quad\left[\mathcal{A}_{2}^{\beta}, \mathcal{A}_{3}^{\beta}\right]=\beta^{2} \mathcal{A}_{1}^{\beta},
$$

which converges to $\left\{\mathcal{A}_{1}, \mathcal{A}_{2}, \mathcal{A}_{3}\right\}=\left\{\partial_{\theta}, \partial_{\xi}, \partial_{\eta}\right\}$ for $\beta \rightarrow 0$. Now the Lie algebra of $S O(3)$ is equal to $s o(3)=\left\{A \in G L(3, \mathbb{R}) \mid A^{T}=-A\right\}$, which is isomorphic to $\mathbb{R}^{3}$ by means of $\mathbf{a} \leftrightarrow(\mathbf{x} \mapsto \mathbf{a} \times \mathbf{x})$, and by direct computation of (3.5) we indeed find $g_{i j}^{\beta=1}=\delta_{i j}$ and (3.8). Moreover (3.9) follows by Cartan's formula [1] for the Riemannian curvature tensor components on compact, semisimple Lie groups: $R_{j, k l}^{i}=\frac{1}{2} \sum_{\lambda} c_{\lambda j}^{i} c_{k l}^{\lambda}$, where $-c_{13}^{2}=c_{31}^{2}=c_{12}^{3}=-c_{21}^{3}=1$ and $c_{23}^{1}=\beta^{2}$. As a matrix group is compact and semisimple iff its Killing form is negative definite, [24, p.269], $(S E(2))_{\beta}$ is compact iff $\beta>0$. However, Cartan's formula also applies to the case $\beta=0$ as we show in Appendix B.

Lemma 3.3. Let $\Phi$ be a mapping on the space of orientation scores $\Phi: \mathbb{C}_{K}^{S E(2)} \rightarrow \mathbb{C}_{K}^{S E(2)}$. Let $\Upsilon_{\psi}=\mathcal{W}_{\psi}^{*} \circ \Phi \circ \mathcal{W}_{\psi}$ be the corresponding operator on the space $\mathbb{L}_{2}\left(\mathbb{R}^{2}\right)$ of images. Let $\mathcal{U}$, respectively $\mathcal{L}$, denote the regular representation of $S E(2)$ onto $\mathbb{L}_{2}\left(\mathbb{R}^{2}\right)$ and $\mathbb{L}_{2}(S E(2)$, given by, respectively, (1.1) and $\mathcal{L}_{g} U(h)=U\left(g^{-1} h\right)$. Then,

$$
\begin{aligned}
& \forall_{g \in S E(2)}: \Phi \circ \mathcal{L}_{g}=\mathcal{L}_{g} \circ \Phi \quad \Leftrightarrow \quad \forall_{g \in S E(2)}: \mathcal{U}_{g} \circ \Upsilon_{\psi}=\Upsilon_{\psi} \circ \mathcal{U}_{g}, \\
& \forall_{g \in S E(2)}: \Phi \circ \mathcal{R}_{g}=\mathcal{R}_{g} \circ \Phi \Leftrightarrow \forall_{g \in S E(2)}: \Upsilon_{\mathcal{U}_{g} \psi}=\Upsilon_{\psi} .
\end{aligned}
$$

\footnotetext{
${ }^{5}$ These spaces are symmetric with respect to fundamental reflection $h \mapsto g h^{-1} g$, 24.
} 


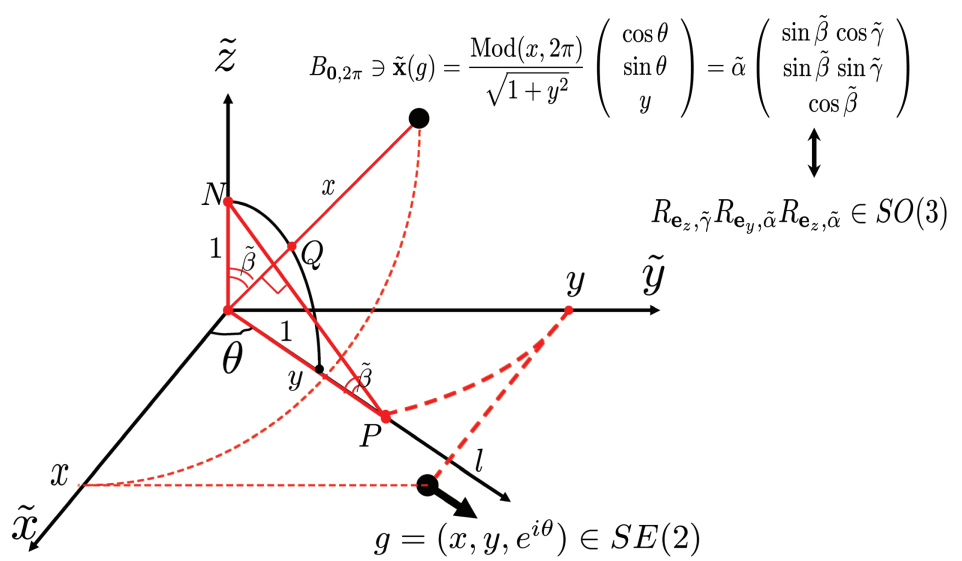

FIG. 4. The embedding of $S E(2)$ in $S O(3)$ by (3.6) for $\beta=1$. The group $S O(3)$ can be identified with a unit ball $B_{\mathbf{0}, 2 \pi}$ with radius $2 \pi$ by means of the Euler angle parametrization. Here all points on the sphere $\left\{\tilde{\mathbf{x}} \in \mathbb{R}^{3} \mid\|\tilde{\mathbf{x}}\|=\tilde{\alpha}=2 \pi\right\}$ are identified with the origin. Now given a fixed member of $\left(x, y, e^{i \theta}\right) \in S E(2)$ we can obtain the corresponding element in $S O(3)$ as follows. First consider the point $(\tilde{x}, \tilde{y}, \tilde{z})=(x, y, 0)$ with attached direction $\theta=\angle((\tilde{x}, \tilde{y}),(1,0))$ and construct the unique half-line $\ell$ through the origin with direction $\theta$. Then project $(x, y, 0)$ on the $\tilde{y}$-axis and rotate the point so that it ends up at point $P$ at the line $\ell$. Then find the unique point $Q$ on the unit sphere such that $\overrightarrow{O Q} \perp \overrightarrow{P N}$ where $N$ is the north pole. Finally, scale $Q$ with $x$ modulo $2 \pi$.

Proof. Recall from Figure1 1 that every operator $\Phi$ on (and in) the space of orientation scores (associated to kernel $\psi$ ) is 1-to-1 related to an operator $\Upsilon_{\psi}=\mathcal{W}_{\psi}^{*} \circ \Phi \circ \mathcal{W}_{\psi}$ on the space of images. Now it is easily verified by conjugation that the following relations hold:

$$
\begin{aligned}
& \forall_{g \in S E(2)}: \mathcal{W}_{\psi} \mathcal{U}_{g}=\mathcal{L}_{g} \mathcal{W}_{\psi} \Leftrightarrow \forall_{g \in S E(2)}: \mathcal{W}_{\psi}^{*} \mathcal{L}_{g}=\mathcal{U}_{g} \mathcal{W}_{\psi}^{*}, \\
& \forall_{g \in S E(2)}: \mathcal{R}_{g} \mathcal{W}_{\psi}=\mathcal{W}_{\mathcal{U}_{g} \psi} \Leftrightarrow \forall_{g \in S E(2)}: \mathcal{W}_{\psi}^{*}=\left(\mathcal{W}_{\mathcal{U}_{g} \psi}\right)^{*} \mathcal{R}_{g},
\end{aligned}
$$

where we used $\left(\mathcal{L}_{g}\right)^{*}=\left(\mathcal{L}_{g}\right)^{-1}=\mathcal{L}_{g^{-1}},\left(\mathcal{R}_{g}\right)^{*}=\left(\mathcal{R}_{g}\right)^{-1}=\mathcal{R}_{g^{-1}},\left(\mathcal{U}_{g}\right)^{*}=\left(\mathcal{U}_{g}\right)^{-1}=\mathcal{U}_{g^{-1}}$ for all $g \in S E(2)$. Now the result (3.11) directly follows from (3.12).

Corollary 3.4. Operators on orientation scores $\Phi$ should be left-invariant (i.e. $\mathcal{L}_{g} \circ \Phi=$ $\left.\Phi \circ \mathcal{L}_{g}\right)$ and not right-invariant in order to ensure that the effective operator $\Upsilon_{\psi}$ is a Euclidean invariant operator which requires an appropriately centered and rotated anisotropic kernel $\psi$.

Therefore we consider the Maurer-Cartan form on $S E(2)$, see Theorem 3.8 and impose the following left-invariant, first fundamental form $\mathcal{G}_{\beta}: S E(2) \times T(S E(2)) \times T(S E(2)) \rightarrow$ $\mathbb{C}$ on $S E(2)$,

$$
\mathcal{G}_{\beta}=\sum_{i, j=1}^{3} g_{i j} \mathrm{~d} \mathcal{A}^{i} \otimes \mathrm{d} \mathcal{A}^{j}=\mathrm{d} \theta \otimes \mathrm{d} \theta+\beta^{2} \mathrm{~d} \mathcal{A}^{2} \otimes \mathrm{d} \mathcal{A}^{2}+\beta^{2} \mathrm{~d} \mathcal{A}^{3} \otimes \mathrm{d} \mathcal{A}^{3},
$$


where $\left[g_{i j}\right]=\operatorname{diag}\left\{1, \beta^{2}, \beta^{2}\right\}$. Here the parameter $\beta$ (physical dimension equals $1 /$ [Length]) should be considered as a fundamental parameter which relates distance on the tori $\left\{\left(\mathbf{x}, e^{i \theta}\right) \mid \theta \in[0,2 \pi)\right\}$ to distances in the spatial planes $\left\{\left(\mathbf{x}, e^{i \theta}\right) \mid \mathbf{x} \in \mathbb{R}^{2}\right\}$. We return to this explanation of $\beta$ later when we put an explicit relation to certain geodesics in $S E(2)$ and elastica curves in $\mathbb{R}^{2}$ (where $\beta^{2}$ determines the typical energy ratio of bending and stretching of an elastic rod).

Remarkably, the left-invariant metric (induced by) $\mathcal{G}_{\beta}$ on $S E(2)$, which serves as the major ingredient in our diffusion schemes on orientation scores in Section 4, is clearly related to the bi-invariant metric (induced by) $\mathcal{G}^{\beta}$ on the compact group $(S E(2))^{\beta}$, (3.8).

In order to be able to understand the full meaning of the next two theorems we need some basic definitions from differential geometry.

Definition 3.5. Let $M$ be a smooth manifold, $G$ be a Lie group. A principal fiber bundle $P_{G}:=(P, M, \pi, R)$ above a manifold $M$ with structure group $G$ is a tuple $(P, M, \pi, R)$ such that $P$ is a smooth manifold, $\pi: P \rightarrow M$ is a smooth projection map with $\pi(P)=M, R$ a smooth right action $R_{g} p=p \cdot g, p \in P, g \in G$, such that $p \cdot(g h)=(p \cdot g) \cdot h$ and $\pi(p \cdot g)=\pi(p)$ for all $p \in P, g, h \in G$. Finally it should satisfy the "local triviality" condition, 34, pp. 346-347].

Definition 3.6. It is common to equip a principal fiber bundle $P_{G}=(P, M, \pi, R)$ with a Cartan-Ehresmann connection form $\omega$. This is by definition a Lie algebra $T_{e}(G)$ valued 1-form $\omega: P \times T(P) \rightarrow T_{e}(G)$ on $P$ such that

$$
\begin{aligned}
& \omega(\mathrm{d} \mathcal{R}(A))=A \text { for all } A \in T_{e}(G), \\
& \omega\left(\left(R_{h}\right)_{*} \mathcal{A}\right)=\operatorname{Ad}\left(h^{-1}\right) \omega(\mathcal{A}) \text { for all vector fields } \mathcal{A} \text { and all } h \in G .
\end{aligned}
$$

It is also common practice to relate principal fiber bundles to vector bundles. Here one uses an external representation $\rho: G \rightarrow F$ into a finite-dimensional vector space $F$ of the structure group to put an appropriate vector space structure on the fibers $\left\{\pi^{-1}(m) \mid m \in M\right\}$ in the principal fiber bundles.

Definition 3.7. Let $P$ be a principal fiber bundle with finite-dimensional structure group $G$. Let $\rho: G \rightarrow F$ be a representation in a finite-dimensional vector space $F$. Then the associated vector bundle is denoted by $P \times{ }_{\rho} F$ and equals the orbit space under the right action

$$
(P \times F) \times G \rightarrow P \times F \text { given by }((u, X), g) \mapsto(u g, \rho(g) X),
$$

for all $g \in G, X \in F$ and $u \in P$.

For details on the associated fiber bundle, see [32, pp. 123-148], where at the end the author provides a clarifying table of correspondences between $P$ and $P \times{ }_{\rho} F$.

Theorem 3.8. The Maurer-Cartan form $\omega$ on $S E(2)$ is given by

$$
\omega_{g}\left(X_{g}\right)=\sum_{i=1}^{3}\left\langle\left.\mathrm{~d} \mathcal{A}^{i}\right|_{g}, X_{g}\right\rangle A_{i}, \quad X_{g} \in T_{g}(S E(2)),
$$

where $\left\{\mathrm{d} \mathcal{A}^{i}\right\}_{i=1}^{3}$ is given by (3.4) and $A_{i}=\left.\mathcal{A}_{i}\right|_{e} ;$ recall (3.3). It is a Cartan-Ehresmann connection form on the principal fiber bundle $P=(S E(2), e, S E(2), \mathcal{L}(S E(2)))$, where $\pi(g)=e, R_{g} u=u g, u, g \in S E(2)$. Let Ad denote the adjoint action of $S E(2)$ on its own Lie algebra $T_{e}(S E(2))$, i.e. $\operatorname{Ad}(g)=\left(R_{g^{-1}} L_{g}\right)_{*}$, i.e. the push-forward of conjugation. 
Then the adjoint representation of $S E(2)$ on the vector space $\mathcal{L}(S E(2))$ of left-invariant vector fields is given by

$$
\widetilde{\operatorname{Ad}}(g)=\mathrm{d} \mathcal{R} \circ \operatorname{Ad}(g) \circ \omega .
$$

This adjoint representation gives rise to the associated vector bundle $S E(2) \times_{\widetilde{\mathrm{Ad}}} \mathcal{L}(S E(2))$. The corresponding connection form on this vector bundle is given by

$$
\tilde{\omega}=\mathcal{A}_{2} \otimes \mathrm{d} \mathcal{A}^{3} \wedge \mathrm{d} \mathcal{A}^{1}+\mathcal{A}_{3} \otimes \mathrm{d} \mathcal{A}^{1} \wedge \mathrm{d} \mathcal{A}^{2} .
$$

Then $\tilde{\omega}$ yields the following $3 \times 3$-matrix-valued 1-form:

$$
\tilde{\omega}_{j}^{k}(\cdot):=-\tilde{\omega}\left(\mathrm{d} \mathcal{A}^{k}, \cdot, \mathcal{A}_{j}\right), \quad k, j=1,2,3
$$

on the frame bundle, [34, p.353, p.359], where the sections are moving frames [34, p.354]. Let $\left\{\mu_{k}\right\}_{k=1}^{3}$ denote the sections in the tangent bundle $E:=(S E(2), T(S E(2)))$ which coincide with the left-invariant vector fields $\left\{\mathcal{A}_{k}\right\}_{k=1}^{3}$. Then the matrix-valued 1-form (3.18) yields the Cartan connection 6 on the tangent bundle $(S E(2), T(S E(2)))$ given by the covariant derivatives

$$
\begin{aligned}
D_{\left.X\right|_{\gamma(t)}}(\mu(\gamma(t))) & :=D \mu(\gamma(t))\left(\left.X\right|_{\gamma(t)}\right) \\
& =\sum_{k=1}^{3} \dot{a}^{k}(t) \mu_{k}(\gamma(t))+\sum_{k=1}^{3} a^{k}(\gamma(t)) \sum_{j=1}^{3} \tilde{\omega}_{k}^{j}\left(\left.X\right|_{\gamma(t)}\right) \mu_{j}(\gamma(t)) \\
& =\sum_{k=1}^{3} \dot{a}^{k}(t) \mu_{k}(\gamma(t))+\sum_{i, j=1}^{3} \dot{\gamma}^{i}(t) a^{k}(\gamma(t)) \Gamma_{i k}^{j} \mu_{j}(\gamma(t)),
\end{aligned}
$$

with $\dot{a}^{k}(t)=\dot{\gamma}^{i}(t)\left(\left.\mathcal{A}_{i}\right|_{\gamma(t)} a^{k}\right)$, for all tangent vectors $\left.X\right|_{\gamma(t)}=\left.\dot{\gamma}^{i}(t) \mathcal{A}_{i}\right|_{\gamma(t)}$ along a curve $t \mapsto \gamma(t) \in S E(2)$ and all sections $\mu(\gamma(t))=\sum_{k=1}^{3} a^{k}(\gamma(t)) \mu_{k}(\gamma(t))$. The Christoffel symbols in (3.19) are constants $\Gamma_{i k}^{j}=-c_{i k}^{j}$, with $c_{i k}^{j}$ the structure constants of the Lie algebra $T_{e}(S E(2))$. The curvature tensor equals

$$
D^{2}=R_{i, k l}^{j} \mathrm{~d} \mathcal{A}^{i} \otimes \mathrm{d} \mathcal{A}^{k} \otimes \mathrm{d} \mathcal{A}^{l} \otimes \mathcal{A}_{j}=\mathcal{A}_{2} \otimes \mathrm{d} \theta \otimes \mathrm{d} \theta \wedge \mathrm{d} \mathcal{A}^{2}+\mathcal{A}_{3} \otimes \mathrm{d} \theta \otimes \mathrm{d} \theta \wedge \mathrm{d} \mathcal{A}^{3} .
$$

This formula arises from (3.9) by taking the limit $\beta \downarrow 0$. So the curvature on $S E(2)$ is constant, the sectional curvature of the planes spanned, respectively, by $\left\{\partial_{\theta}, \partial_{\xi}\right\}$ and by $\left\{\partial_{\theta}, \partial_{\eta}\right\}$ is 1 (so constant) and the sectional curvature of the plane spanned by $\left\{\partial_{\xi}, \partial_{\eta}\right\}$ vanishes.

Proof. For the proof, see Appendix B.

The next theorem relates the previous results on Cartan connections and covariant derivatives to our nonlinear diffusion schemes on $S E(2)$.

Theorem 3.9. The covariant derivative of a co-vector field $\mathbf{a}$ on the manifold $\left((S E(2))^{\beta}\right.$, $\left.\mathcal{G}^{\beta}\right)$ is a $(0,2)$-tensor field with components: $\nabla_{j} a_{i}=\mathcal{A}_{j} a_{i}-\Gamma_{j i}^{k} a_{k}$, whereas the covariant derivative of a vector field $\mathbf{v}$ on $(S E(2))^{\beta}$ is a $(1,1)$-tensor field with components $\nabla_{j^{\prime}} v^{i}=$ $\mathcal{A}_{j^{\prime}} v^{i}+\Gamma_{j^{\prime} k^{\prime}}^{i} k^{k^{\prime}}$. Here the Christoffel symbols equal minus the structure constants of

\footnotetext{
${ }^{6}$ Following the definitions in [34, it is formally not right to call this the Cartan connection. It is the Koszul connection, 34 p.242], corresponding to the Cartan connection, 34 p.353], i.e. the associated differential operator corresponding to a Cartan connection. For a complete overview on Koszul connections, Ehresmann connections (the most general ones), Cartan connections and classical connections, see [34 pp. 386-387]. From now on we avoid all these technicalities and just use "Cartan connection" (a Koszul connection in 34]) and "Cartan-Ehresmann connection form" (Ehresmann connection in 34]).
} 
the Lie algebra $\mathcal{L}\left((S E(2))^{\beta}\right): \Gamma_{i j}^{k}=-c_{i j}^{k}$ using short notation $\nabla_{j}:=D_{\mathcal{A}_{j}}$, where $D$ denotes the Cartan connection on $(S E(2))_{\beta}$. The Christoffel symbols are anti-symmetric as the underlying Cartan connection $D$ has constant curvature and constant torsion. The left-invariant evolution equations (1.9) can be rewritten in covariant derivatives:

$$
\left\{\begin{array}{l}
\partial_{s} W(g, s)=\sum_{i, j=1}^{3} \mathcal{A}_{i}\left(\left(D_{i j}(W)\right)(g, s) \mathcal{A}_{j} W\right)(g, s)=\sum_{i, j=1}^{3} \nabla_{i}\left(\left(D_{i j}(W)\right)(g, s) \nabla_{j} W\right)(g, s), \\
W(g, 0)=\mathcal{W}_{\psi} f(g), \quad \text { for all } g \in S E(2), s>0 .
\end{array}\right.
$$

Both convection and diffusion in the left-invariant evolution equations (1.9) take place along the exponential curves in $S E(2)$ which are the covariantly constant curves with respect to the Cartan connection. These curves are circular spirals in $\mathbb{R}^{2} \times[0,2 \pi)$ :

$$
\begin{aligned}
& t \mapsto g_{0} \exp \left(t\left(\sum_{i=1}^{3} c_{i} A_{i}\right)\right)=\left(x_{0}+\frac{c_{3}}{c_{1}}\left(\cos \left(c_{1} t+\theta_{0}\right)-\cos \theta_{0}\right)+\frac{c_{2}}{c_{1}}\left(\sin \left(c_{1} t+\theta_{0}\right)-\sin \theta_{0}\right),\right. \\
& \left.y_{0}+\frac{c_{3}}{c_{1}}\left(\sin \left(c_{1} t+\theta_{0}\right)-\sin \theta_{0}\right)-\frac{c_{2}}{c_{1}}\left(\cos \left(c_{1} t+\theta_{0}\right)-\cos \theta_{0}\right), e^{i\left(c_{1} t+\theta_{0}\right)}\right), \quad c_{1} \neq 0,
\end{aligned}
$$

for all $g_{0}=\left(x_{0}, y_{0}, e^{i \theta_{0}}\right) \in S E(2)$, with radius $\frac{\sqrt{c_{2}^{2}+c_{3}^{2}}}{c_{1}}$ and central point $\left(-\frac{c_{3}}{c_{1}} \cos \theta_{0}-\right.$ $\left.\frac{c_{2}}{c_{1}} \sin \theta_{0}+x_{0}, \frac{c_{2}}{c_{1}} \cos \theta_{0}-\frac{c_{3}}{c_{1}} \sin \theta_{0}+y_{0}\right)$. For $c_{1}=0$ the exponential curves are given by

$t \mapsto g_{0} \exp \left(t\left(c_{2} A_{2}+c_{3} A_{3}\right)\right)=\left(x_{0}+t c_{2} \cos \theta_{0}-t c_{3} \sin \theta_{0}, y_{0}+t c_{2} \sin \theta_{0}+t c_{3} \cos \theta_{0}, e^{i \theta_{0}}\right)$.

Proof. The first part of the proof is a straightforward generalization of Theorem 3.8, where $\beta=0$. Here we note that by Theorem 3.2 covariant differentiation takes place on a symmetric Riemannian manifold $\left((S E(2))_{\beta}\right), \mathcal{G}_{\beta}$ iff $\beta>0$. We know by Theorem 3.2 that the curvature of the Cartan connection $D$ on $(S E(2))_{\beta}$ is constant. Now the torsion tensor $T(X, Y)=D_{X} Y-D_{Y} X-[X, Y]$ is constant as well, since $T\left[\mathcal{A}_{i}, \mathcal{A}_{j}\right]=$ $D_{\mathcal{A}_{i}} \mathcal{A}_{j}-D_{\mathcal{A}_{j}} \mathcal{A}_{i}-\left[\mathcal{A}_{i}, \mathcal{A}_{j}\right]=\sum_{k}\left(\Gamma_{i j}^{k} \mathcal{A}_{k}-\Gamma_{j i}^{k} \mathcal{A}_{k}-c_{i j}^{k} \mathcal{A}_{k}\right)=-3 \sum_{k} c_{i j}^{k} \mathcal{A}_{k}$. Finally, the covariant constant curves $\gamma$ (or "auto-parallel" curves) are by definition given by $D_{\dot{\gamma}} \dot{\gamma}=0$ on the tangent bundle $(S E(2), T(S E(2)))$ :

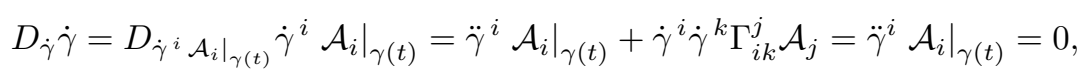

where we again apply automatic summation over double indices and where $\Gamma_{i j}^{k}=-\Gamma_{j i}^{k}=$ $c_{j i}^{k}=-c_{i j}^{k}$. Apparently, tangent vectors to auto-parallel curves have constant coefficients with respect to $\left\{\mathcal{A}_{1}, \mathcal{A}_{2}, \mathcal{A}_{3}\right\}$, i.e. $\forall_{t>0} \dot{\gamma}^{i}(t)=\left\langle\left.\mathrm{d} \mathcal{A}^{i}\right|_{\gamma(t)}, \dot{\gamma}(t)\right\rangle=\left\langle\left.\mathrm{d} \mathcal{A}^{i}\right|_{\gamma(0)}, \dot{\gamma}(0)\right\rangle=c^{i} \in$ $\mathbb{R}, i=1,2,3$. Now for smooth $U: S E(2) \rightarrow \mathbb{C}$ one has

$$
\begin{aligned}
& \frac{d}{d t} U(\gamma(t))=\lim _{h \rightarrow 0} \frac{U(\gamma(t+h)-U(\gamma(t)))}{h}=\left(\mathrm{d} \mathcal{R}\left(\sum_{i=1}^{3} c^{i} A_{i}\right) U\right)(\gamma(t))=\sum_{i=1}^{3} c^{i}\left(\mathrm{~d} \mathcal{R}\left(A_{i}\right) U\right)(\gamma(t)) \\
& =\left.\sum_{i=1}^{3} c^{i} \mathcal{A}_{i} U\right|_{\gamma(t)}, \text { where } \gamma(t)=g_{0} e^{t \sum_{i=1}^{3} c^{i} A_{i}}, \text { recall } A_{i}=\left.\mathcal{A}_{i}\right|_{e},
\end{aligned}
$$

so these curves $\gamma(t)$ coincide with the exponential curves in $S E(2)$, derived in [15, 11]. Now the connection $D$ is a Koszul connection, [34, p.241], and therefore $\nabla_{i}\left(U \mathcal{A}_{j}\right) \phi=$ $\nabla_{i}\left(U \nabla_{j}\right) \phi=U \nabla_{i} \nabla_{j} \phi+\left(\nabla_{i} U\right) \nabla_{j} \phi$ for all $U \in C^{1}(S E(2))$ and all smooth $\phi \in$ $C^{\infty}(S E(2))$. Now set $U=D_{i j}(W)(\cdot, s)$ and $\phi=W(\cdot, s)$ for all $s>0$, use $D_{i j}=D_{j i}$ and $\Gamma_{i j}^{k}=-\Gamma_{j i}^{k}$, take the sum over both indices $i, j$ and the result (3.21) follows.

\footnotetext{
${ }^{7}$ Our Cartan connection is not related to a Levi-Civita connection (where Christoffels are symmetric).
} 
REMARK 3.10. Circular spirals are the only curves with nonzero constant curvature and torsion in the flat space $\mathbb{R}^{2} \times[0,2 \pi)$. A circular spiral is covariantly constant in $(S E(2), T(S E(2)))$ with respect to the Cartan connection with constant curvature and torsion iff the principal axis of the cylinder containing the spiral lies in the $\theta$-direction.

3.3. Horizontal curves and principal fiber bundles. There exists a natural relation between curves in the plane $\mathbb{R}^{2}$ and curves in $S E(2)$. For every $C^{1}$-curve $s \mapsto(x(s), y(s))$ one can construct a unique corresponding curve in $S E(2)$ by $s \mapsto(x(s), y(s), \theta(s)=$ $\left.\arg \left(x^{\prime}(s)+i y^{\prime}(s)\right)\right)$. We will call such curves in $S E(2)$ horizontal curves.

Definition 3.11. A curve $s \mapsto \gamma(s)=\left(x(s), y(s), e^{i \theta(s)}\right)$ in $S E(2)$ is called horizontal iff $\theta(s)=\arg \left(x^{\prime}(s)+i y^{\prime}(s)\right)$. Then $\gamma$ is called the lifted curve in $S E(2)$ of the curve $s \mapsto \mathbf{x}(s)=(x(s), y(s))$ in $\mathbb{R}^{2}$. A tangent vector in $X_{g} \in T_{g}(S E(2))$ is called horizontal if it is the tangent vector to some horizontal curve through $g$. A vector field $X$ is called horizontal if $X_{g}$ is horizontal for all $g \in S E(2)$.

We want to diffuse on orientation scores mainly along horizontal exponential curves; for a practical motivation, see [20. To get the right intuition: Recall [17, Fig.1], where typically the mass of an orientation score is concentrated around a horizontal curve.

A smooth horizontal curve $\gamma=\left(\mathbf{x}, e^{i \theta}\right)$ in $S E(2)$, given by $s \mapsto\left(\mathbf{x}(s), e^{i \theta}(s)\right)$, can be parameterized by the arc-length $s>0$ of its projection $\mathbf{x}=\mathbb{P}_{\mathbb{R}^{2}} \gamma$ on the spatial plane. Using this spatial arc-length parametrization it is clear from Definition 3.11 that along a horizontal curve $\gamma=\left(\mathbf{x}, e^{i \theta}\right): \mathbb{R}^{+} \rightarrow S E(2)$ one has

$$
\gamma=\left(\mathbf{x}, e^{i \theta}\right) \text { is horizontal } \Rightarrow \kappa(s)=\dot{\theta}(s),
$$

where $\kappa(s)= \pm\|\ddot{\mathbf{x}}(s)\|_{\mathbb{R}^{2}}$, with $\|\cdot\|_{\mathbb{R}^{2}}$ the Euclidean norm on $\mathbb{R}^{2}$, is the curvature of the curve $s \mapsto \mathbf{x}(s)=\mathbb{P}_{\mathbb{R}^{2}} \gamma(s)$. Furthermore, a smooth curve $s \mapsto \gamma(s)$ is horizontal iff

$$
\dot{\gamma}(s) \in \operatorname{span}\left\{\left.\mathbf{e}_{\theta}\right|_{\gamma(s)},\left.\mathbf{e}_{\xi}\right|_{\gamma(s)}\right\} \text { for all } s>0 .
$$

Here we denote tangent vectors as $\mathbf{e}_{\theta}(s)=\mathbf{e}_{\theta}, \mathbf{e}_{\xi}(s)=\cos \theta(s) \mathbf{e}_{x}+\sin \theta(s) \mathbf{e}_{y}, \mathbf{e}_{\eta}(s)=$ $-\sin \theta(s) \mathbf{e}_{x}+\cos \theta(s) \mathbf{e}_{y}$ as they are considered as tangent vectors to (classes of) curves, rather than considering tangent vectors as differential operators $\left\{\partial_{\theta}, \partial_{\xi}, \partial_{\eta}\right\}$ on locally defined smooth functions, although by the isomorphisms described in [4] it boils down to the same thing; recall [17, Fig.4].

By equality (3.26) and Definition 3.11 it follows that the horizontal part $\mathcal{H}_{g} \subset$ $T_{g}(S E(2))$ of each tangent space $T_{g}(S E(2))$ is spanned by $\mathcal{H}_{g}=\left\{\left.\partial_{\theta}\right|_{g},\left.\partial_{\xi}\right|_{g}\right\}$, and the space of horizontal left-invariant vector fields is spanned by $\left\{\partial_{\theta}, \partial_{\xi}\right\}=\left\{\mathcal{A}_{1}, \mathcal{A}_{2}\right\}$. However, a horizontal curve itself $s \mapsto \gamma(s) \in S E(2)$ can have components in all directions $\left\{\mathbf{e}_{\theta}, \mathbf{e}_{\xi}, \mathbf{e}_{\eta}\right\}$, in contrast to $\dot{\gamma}(s) \in T_{\gamma(s)}(S E(2))$. In fact a smooth curve $\gamma$ given by

$$
s \mapsto \gamma(s)=\xi(s) \mathbf{e}_{\xi}(s)+\eta(s) \mathbf{e}_{\eta}(s)+\theta(s) \mathbf{e}_{\theta}(s),
$$

with $\gamma(s)=\left(x(s), e^{i \theta(s)}\right) \in S E(2)$ and $s>0$ the arc length of the projected curve $x=\mathbb{P}_{\mathbb{R}^{2}} \gamma$, is a horizontal curve in $S E(2)$ iff $\frac{d \eta}{d s}=-\xi \kappa$. Moreover, for such curves we have $\frac{d \xi}{d s}-\kappa \eta=\|\dot{x}(s)\|=1$. This follows by differentiation of $s \mapsto \gamma(s)$ and observation (3.26):

$$
\frac{d}{d s}\left(\xi(s) \mathbf{e}_{\xi}(s)+\eta(s) \mathbf{e}_{\eta}(s)+\theta(s) \mathbf{e}_{\theta}\right)=(\dot{\xi}(s)-\kappa(s) \eta(s)) \mathbf{e}_{\xi}(s)+(\dot{\eta}(s)+\kappa(s) \xi(s)) \mathbf{e}_{\eta}(s)+\dot{\theta}(s) \mathbf{e}_{\theta} .
$$


Differentiating a smooth function $C: S E(2) \rightarrow \mathbb{R}$ along a horizontal curve $\gamma$ yields

$$
\begin{aligned}
\frac{d}{d s} C(\gamma(s)) & =\left\langle C_{\xi}(\gamma(s)) \mathrm{d} \mathcal{A}^{2}+C_{\eta}(\gamma(s)) \mathrm{d} \mathcal{A}^{3}+C_{\theta}(\gamma(s)) \mathrm{d} \theta, \dot{\gamma}(s)\right\rangle \\
& =\left(C_{\xi}(\gamma(s))\left(\frac{d \xi}{d s}-\kappa(s) \eta(s)\right)+C_{\eta}(\gamma(s))\left(\frac{d \eta}{d s}+\kappa(s) \xi(s)\right)+C_{\theta}(\gamma(s)) \kappa(s)\right) \\
& =C_{\xi}(\gamma(s))+\kappa(s) C_{\theta}(\gamma(s)) .
\end{aligned}
$$

A horizontal curve can be mapped to a new horizontal curve by right multiplication with a fixed element from the subgroup $Y=\left\{\left(0, y, e^{i 0}=1\right) \mid y \in \mathbb{R}\right\}$. Here we note that

$$
\gamma(s)(0, y, 1)=\left(\mathbf{x}(s), e^{i \theta(s)}\right)(0, y, 1)=\left(\mathbf{x}(s)+y \mathbf{e}_{\eta}(s), e^{i \theta(s)}\right)
$$

and $\dot{\mathbf{x}}^{N E W}(s)=\dot{\mathbf{x}}(s)+\frac{d}{d s} \mathbf{e}_{\eta}(s)=\dot{\mathbf{x}}(s)+\kappa(s) \mathbf{e}_{\xi}(s)=(1+\kappa(s)) \mathbf{e}_{\xi}(s)$, so again the horizontal condition $\theta^{N E W}(s)=\arg \left(\dot{x}^{N E W}(s)+i \dot{y}^{N E W}(s)\right)$ holds and therefore

$$
s \mapsto \gamma(s) \text { is horizontal } \Rightarrow s \mapsto(\gamma(0, h, 0))(s):=\gamma(s)(0, h, 0) \text { is horizontal . }
$$

In fact $Y$ is the only subgroup of $S E(2)$ that has this property, whereas left multiplication of a horizontal curve with any fixed element from $S E(2)$ always yields a horizontal curve again. For an overview of all (length-preserving) perturbations of horizontal curves into horizontal curves, see [16, app. C].

Let us return to our goal of diffusing on orientation scores along horizontal exponential curves; for a practical motivation, see 20 . Now by (3.26) this simply means that in the diffusion generator (1.8) of our nonlinear diffusion system on orientation scores (3.21), all $\partial_{\eta}$ derivatives should be removed (or equivalently set $a_{3}=D_{i 3}=D_{3 i}=0, i=1,2,3$ ). Recall from [17] that this removal does not cause singular behavior (like it would on $\mathbb{R}^{3}$ ) iff $\{1,3\} \in\left\{i \mid a_{i} \neq 0 \vee D_{i i} \neq 0\right\}$ or $\{1,2\} \in\left\{i \mid a_{i} \neq 0 \vee D_{i i} \neq 0\right\}$, because of the noncommutativity of $S E(2)$ and Hörmander's condition, 23. We stress that diffusion does not take place on an integrable 2D submanifold of $S E(2)$ (not even locally !), due to the nonintegrability of the $\left\{\mathrm{d} \mathcal{A}^{1}, \mathrm{~d} \mathcal{A}^{2}\right\}=\{\mathrm{d} \theta, \cos \theta \mathrm{d} x+\sin \theta \mathrm{d} y\}$-foliation, nor does the horizontal diffusion take place on the quotient $S E(2) / Y$, as horizontal curves can have an $\eta$-component in (3.27).

Summarizing, we need a better mathematical grip on the removal of the third direction in the tangent space. Now technically speaking this means that diffusion takes place along the contact manifold $\left\{S E(2), \mathrm{d} \mathcal{A}^{3}\right\}$, 7, p.9]. Here we note that $\mathrm{dd} \theta=0$ and

$$
\begin{array}{ll}
\mathrm{d}(-\sin \theta \mathrm{d} x+\cos \theta \mathrm{d} y) & =-\cos \theta \mathrm{d} \theta \wedge \mathrm{d} x-\sin \theta \mathrm{d} \theta \wedge \mathrm{d} y, \\
\mathrm{~d}(\cos \theta \mathrm{d} x+\sin \theta \mathrm{d} y) & =-\sin \theta \mathrm{d} \theta \wedge \mathrm{d} x+\cos \theta \mathrm{d} \theta \wedge \mathrm{d} y,
\end{array}
$$

which coincides with Cartan's structural formula on $S E(2)$, [1], B.10), in explicit form. For example one has $\mathrm{dd} \mathcal{A}^{3}=-\mathrm{d} \mathcal{A}^{1} \wedge \mathrm{d} \mathcal{A}^{2}$, where in the left-hand side only the left $\mathrm{d}$ denotes an exterior derivative ( $\mathrm{d} \mathcal{A}^{i}$ denotes the dual vector to $\mathcal{A}_{i}$ as in Theorem 3.1). So the nondegeneracy condition on the Pfaffian form $\mathrm{d} \mathcal{A}^{2}$ of the contact manifold [7, p.9] is indeed satisfied : $\mathrm{d} \mathcal{A}^{3} \wedge \mathrm{dd} \mathcal{A}^{3}=\mathrm{d} \mathcal{A}^{1} \wedge \mathrm{d} \mathcal{A}^{2} \wedge \mathrm{d} \mathcal{A}^{3} \neq 0$. It is well known in the theory on contact manifolds [7] that the only integrable submanifolds are one-dimensional and they are usually called Legendre submanifolds, which in our case simply coincide with horizontal curves on $S E(2)$. Contact manifold theory, [6], is highly useful for optimization of Lagrangians along horizontal curves, [7, ch:1.2], as can be seen in Appendix A. But it is mainly based on Pfaffian forms (elements in the dual tangent space) and we rather 
need a fiber structure in the manifold $S E(2)$. Therefore we will use the Pfaffian form $\mathrm{d} \mathcal{A}^{3}$ of the contact manifold in an Ehresmann connection of a principal fiber bundle on $S E(2)$. So we consider the domain of the (evolving) orientation score as the following principal fiber bundle:

$$
P_{Y}=(S E(2), S E(2) / Y, \pi, R),
$$

with subgroup $Y=\left\{\left(0, y, e^{i 0}\right) \mid y \in \mathbb{R}\right\}$, right-multiplication $R_{h} g=g h, h \in Y, g \in S E(2)$ and projection $\pi: S E(2) \rightarrow S E(2) / Y$ given by $\pi(g)=g Y=\left\{g^{\prime} \in S E(2) \mid g^{\prime} \sim\right.$ $g$ i.e. $\left.g^{-1} g^{\prime} \in Y\right\}$, so that $\pi(g h)=\pi(g)$ for all $g \in S E(2), h \in Y$. For more details on principal fiber bundles in general, see [26, 34], 32].

Here we stress that coordinate-free differential geometry on principal fiber bundles starts with horizontal lifts as defined below. These horizontal lifts define parallel transport (which turns out to be independent of the choice of horizontal lift), [34, ch:8, p.365] and parallel transport defines the covariant derivative [34, p.366] which yields a connections, [34, p.367-368].

Definition 3.12. A horizontal lift of a curve $\gamma:[0,1] \rightarrow S E(2) / Y$ is a horizontal curve $\gamma^{*}:[0,1] \rightarrow S E(2)$ such that $\pi\left(\gamma^{*}\right)=\gamma$.

It can be shown [34, Prop. 7, p.363] that for every curve in $\gamma:[0,1] \rightarrow S E(2) / Y$ with $\gamma(0)=g_{0} Y$ for some $g_{0} \in P_{Y}$ meaning $g_{0} \in S E(2)$, say $\pi\left(g_{0}\right)=g_{0} Y$, there exists a unique lift $\gamma^{*}$ of $\gamma$ such that $\gamma^{*}(0)=g_{0}$. In fact horizontal lifts are uniquely determined by right multiplication and since $Y$ is the unique subgroup satisfying (3.30), we are able to relate our basic definition of horizontal vector fields, Definition 3.11 to the standard definition of horizontal vector fields on the principal fiber bundle $P_{Y}$; see Theorem 3.13 .

On $P_{Y}$ one can still impose a left-invariant metric, as is done in Theorem 3.13, again parameterized by the same $\beta>0$, by removing the left-invariant direction $\mathcal{A}_{3}$ from each tangent space. The geodesics on this principal fiber bundle are closely related to elastica curves and are derived in Appendix A. Our formula for these geodesics is much more tangible than the well-known formula for the corresponding elastica curves, [29, [6]. For a comparison between elastica and corresponding geodesics, see [16, Ch: 7, Fig.13].

Theorem 3.13. The set $P_{Y}=(S E(2), S E(2) / Y, \pi, R)$ with subgroup $Y=\left\{\left(0, y, e^{i 0}\right) \mid\right.$ $y \in \mathbb{R}\}$, projection $\pi(g)=g Y$ and right-multiplication $R_{h} g=g h, h \in Y$, is a principal fiber bundle (with structure group $Y$ ) on which $\omega=\left(L_{0,-y, 0}\right)_{*}$ is a Cartan-Ehresmann connection form and, in the moving frame of reference $\left\{\mathcal{A}_{1}, \mathcal{A}_{2}, \mathcal{A}_{3}\right\}$, it is given by

$$
\omega_{g}\left(X_{g}\right)=\left\langle\left.\mathrm{d} \mathcal{A}^{2}\right|_{g}, X_{g}\right\rangle A_{2}=\left\langle-\sin \theta \mathrm{d} x+\cos \theta \mathrm{d} y, X_{g}\right\rangle \partial_{y}, \quad X \in \mathcal{L}(S E(2)) .
$$

The horizontal part $\mathcal{H}_{g}$ of each tangent space $T_{g}(S E(2)), g \in S E(2)$, is by definition

$$
\mathcal{H}_{g}:=\operatorname{ker}\left\{\omega_{g}\right\}=\operatorname{span}\left\{\left.\mathcal{A}_{1}\right|_{g},\left.\mathcal{A}_{2}\right|_{g}\right\}=\operatorname{span}\left\{\partial_{\theta}, \cos \theta \partial_{x}+\sin \theta \partial_{y}\right\}
$$

and it coincides with the vector space of tangent vectors along all possible horizontal curves passing through $g \in S E(2)$; recall Definition 3.11. The connection form $\tilde{\omega}$ on the

\footnotetext{
${ }^{8}$ This Koszul connection coincides, [34 pp. 368-371], with the Koszul connection [p.320] 34] obtained from the Cartan connection, [34, ch:7,p.314] corresponding to an Ehresmann connection, [34, p.359] on the frame-bundle $F(S E(2) / Y)$, [34, ch:7,p.345].
} 
associated vector bundle $S E(2) \times_{\widetilde{\mathrm{Ad}}} \mathcal{L}(S E(2))$ is given by

$$
\tilde{\omega}=-\mathcal{A}_{2} \otimes \mathrm{d} \mathcal{A}^{1} \otimes \mathrm{d} \mathcal{A}^{3}=-\left(\cos \theta \partial_{x}+\sin \theta \partial_{y}\right) \otimes \mathrm{d} \theta \otimes(-\sin \theta \mathrm{d} x+\cos \theta \mathrm{d} y) .
$$

The horizontal auto-parallels of the connection $D=d+\bar{\omega}$, with $\bar{\omega}\left(a^{k} \mathcal{A}_{k}\right)=$ $-a^{k} \tilde{\omega}\left(\mathrm{d} \mathcal{A}^{j}, \cdot, \mathcal{A}_{k}\right) \mathcal{A}_{j}$

$$
D_{a^{i} \mathcal{A}_{i}}\left(\left.\dot{\gamma}^{i} \mathcal{A}_{i}\right|_{\gamma(t)}\right)=\left(D a^{i} \mathcal{A}_{i}\right)\left(\left.\dot{\gamma}^{i} \mathcal{A}_{i}\right|_{\gamma(t)}\right)=\left.\dot{a}^{1} \mathcal{A}_{1}\right|_{\gamma(t)}+\left.\left(\dot{a}^{2}+a^{3} \dot{\gamma}^{1}\right) \mathcal{A}_{2}\right|_{\gamma(t)}+\left.\dot{a}^{3} \mathcal{A}_{3}\right|_{\gamma(t)},
$$

are the horizontal exponential curves, given by (3.22) with $c_{3}=0$; see Figure 5 ,

Finally, we equip $P_{Y}$ with the following left-invariant form:

$$
\mathcal{G}_{\beta}=\mathrm{d} \theta \otimes \mathrm{d} \theta+\beta^{2} \mathrm{~d} \mathcal{A}^{2} \otimes \mathrm{d} \mathcal{A}^{2},
$$

which yields a left-invariant metric on $S E(2): d_{S E(2)}\left(g, g_{0}\right)=d_{S E(2)}\left(g_{0}^{-1} g, e\right)$ defined by

$$
\begin{aligned}
& d_{S E(2)}\left(g_{0}^{-1} g, e\right)=\inf \left\{\int_{0}^{1} \sqrt{\left(\theta^{\prime}(t)\right)^{2}+\beta^{2}\left\|\mathbf{x}^{\prime}(t)\right\|^{2}} \mathrm{~d} t \mid \gamma \text { horizontal, } \gamma(0)=e, \gamma(L)=g_{0}^{-1} g\right\} \\
& =\inf \left\{\int_{0}^{L} \sqrt{(\kappa(s))^{2}+\beta^{2}} \mathrm{~d} s=\int_{0}^{L} \sqrt{g_{i j} \dot{\gamma}^{i}(s) \dot{\gamma}^{j}(s)} \mathrm{d} s \mid \gamma \text { horizontal, } \gamma(0)=e, \gamma(L)=g_{0}^{-1} g\right\},
\end{aligned}
$$

where $s>0$ denotes the spatial arc-length parameter of the projected curve $\mathbf{x}=\mathbb{P}_{\mathbb{R}^{2}} \gamma$, with curvature $\kappa(s)=\|\ddot{\mathbf{x}}(s)\|$ in $\mathbb{R}^{2}$ and where $\dot{\gamma}^{i}(s)=\left\langle\mathrm{d} \mathcal{A}^{i}, \dot{\gamma}(s)\right\rangle, i=1,2,3$. The explicit curves that minimize (3.38) are explicitly derived in Appendix A In contrast to previous belief, [9], they do not exactly coincide with elastica curves, [29, [6].

Proof. The first part of the proof is analogous to the proof of Theorem 3.8 in Appendix B. The big difference, though, is that instead of a principal fiber bundle $P$ with structure group $S E(2)$ we now have fiber bundle $P_{Y}$ with structure group $Y$. In particular the base manifold $\{e\} \equiv S E(2) / S E(2)$ is now replaced by $S E(2) / Y$. In Theorem 3.8 every tangent vector is vertical, whereas in this theorem we are rather interested in the horizontal part $\mathcal{H}_{g}$ of the tangent space $T(S E(2)$. Note that by (3.26) the differential geometrical definition of horizontality $\mathcal{H}_{g}:=\operatorname{ker}\left\{\omega_{g}\right\}$ coincides with the horizontality condition, Definition 3.11, required in the application! The connection form $\omega_{g}$ is indeed a CartanEhresmann connection form on $P_{Y}$ : The first condition in Definition 3.6 is satisfied since $\omega \circ \mathrm{d} \mathcal{R}\left(A_{3}\right)=\omega\left(\mathcal{A}_{3}\right)=A_{3}$, and the second condition follows by (3.14) (special case $g \in Y)$.

Again the Cartan connection $D$ is obtained via the connection form $\tilde{\omega}$. This is the corresponding connection form on the associated vector bundle $S E(2) \times \widetilde{\mathrm{Ad}} \mathcal{L}(S E(2))$ given by $\tilde{\omega}=\widetilde{\operatorname{Ad}}_{*}\left(A_{3}\right) \otimes \mathrm{d} \mathcal{A}^{3}=\widetilde{\operatorname{ad}}\left(\mathcal{A}_{3}\right) \otimes \mathrm{d} \mathcal{A}^{3}=c_{i 3}^{k} \mathcal{A}_{k} \otimes \mathrm{d} \mathcal{A}^{i} \otimes \mathrm{d} \mathcal{A}^{3}=c_{13}^{2} \mathcal{A}_{2} \otimes \mathrm{d} \mathcal{A}^{1} \otimes \mathrm{d} \mathcal{A}^{3}$, yielding (3.35). Here we note that direct computation yields $\bar{\omega}\left(a^{1} \mathcal{A}_{1}+a^{2} \mathcal{A}_{2}+a^{3} \mathcal{A}_{3}\right)=$ $a^{3} \mathrm{~d} \mathcal{A}^{1}(\cdot) \mathcal{A}_{2}$ from which (3.36) directly follows. Tangent vectors $\dot{\gamma}=\left.\sum_{i} \dot{\gamma}^{i}(t) \mathcal{A}_{i}\right|_{\gamma(t)}$ to auto-parallel curves satisfy: $\nabla_{\dot{\gamma}} \dot{\gamma}=0 \Leftrightarrow \ddot{\gamma}^{2}=-\dot{\gamma}^{1} \dot{\gamma}^{3}$ and $\ddot{\gamma}^{1}=\ddot{\gamma}^{3}=0$, so in particular if they are horizontal we find $\ddot{\gamma}^{2}=\ddot{\gamma}^{1}=\dot{\gamma}^{3}=0$. As a result all auto-parallel curves in the fiber bundle are horizontal exponential curves (3.22) with constants $c_{1}=\dot{\gamma}^{1}, c_{2}=\dot{\gamma}^{2}$, $c_{3}=0$. Now (3.37) follows from (3.13) by omitting the vertical direction $\mathcal{A}_{3}$ and in (3.38) we stress that the Lagrangian is parameter independent, so we may as well choose the spatial arc-length parameter $s>0$ of the projection of the horizontal curve on the spatial plane with length $L$, in which case we have $\kappa(s)=\dot{\theta}(s)$. The metric (3.37) coincides with the metric in [9] (where this metric is called an "elastica functional") and is related to the well-known elastica functional $\int_{0}^{L} \kappa^{2}(s)+\beta^{2} \mathrm{~d} s,[29$, [6], for curves 

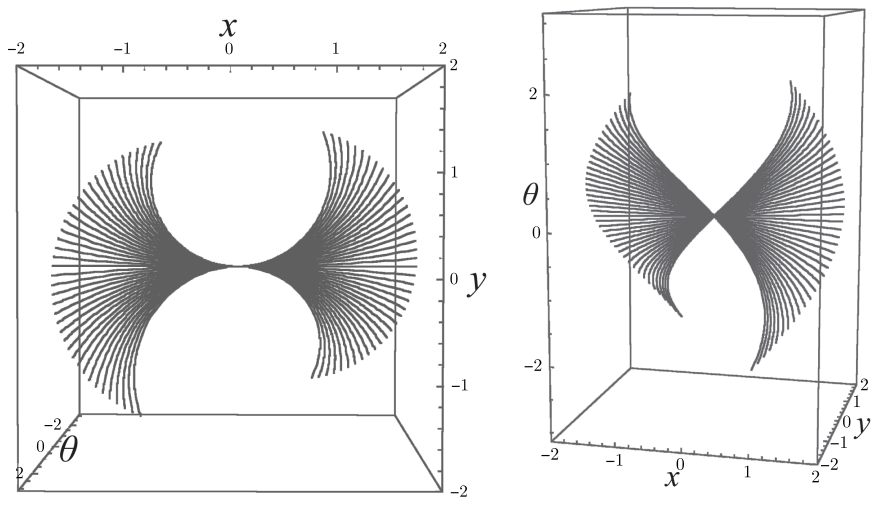

Fig. 5. All horizontal exponential curves through a fixed point $g \in S E(2)$ for different curvature values, shown from different viewpoints. The left image shows that these curves correspond to circular arcs if projected onto the spatial plane. These curves coincide with the auto-parallels, see (3.22), in the principal fiber bundle $P_{Y}$.

in $\mathbb{R}^{2}$. The important difference though is the square root! This square root ensures that the functional is parameter independent in $S E(2)$, whereas the elastica functional is only parameter independent on $\mathbb{R}^{2}$. In a standard Riemannian manifold it does not matter if one applies a monotonic transformation on the integrand of the metric (this monotonic transformation can be taken into account by a reparametrization of the same curve). However this argument does not apply here since only for the spatial arc-length parametrization of a horizontal curve do we have $\kappa(s)=\dot{\theta}(s)$.

Finally we note with respect to the fundamental parameter $\beta^{2}$, which in the case of elastica denotes the typical fraction of bending and stretching energy, that there exist two fundamentally different approaches to relate minimal energy curves to direct products of Green's functions of stochastic processes on $S E(2)$ with linear forward Kolmogorov equations, parameterized by constants $\alpha>0,\left[D_{i j}\right] \geq 0$ as considered in part I [17]. If one follows the approach by Mumford, 29], to relate elastica curves to the direction process (a contour-completion process), then one must set $\beta^{2}=4 \alpha D_{11}$; if one follows Brownian bridge theory, [16, app.B], to relate geodesics to a contour-enhancement process with $D_{i j} \neq 0 \Leftrightarrow(i=j=1$ or $i=j=2)$, then one takes the limit $\alpha^{-1} \rightarrow 0$ and sets $\beta^{2}=D_{11} / D_{22}$.

3.4. Extraction of spatial curvature from orientation scores. Let $U: S E(2) \rightarrow \mathbb{R}^{+}$be some positive smooth function on $S E(2)$. This could for example be the absolute value $U=\left|\mathcal{W}_{\psi} f\right|=\sqrt{\left(\Re\left(\mathcal{W}_{\psi} f\right)\right)^{2}+\left(\Im\left(\mathcal{W}_{\psi} f\right)\right)^{2}}$ of a (processed) orientation score of an image, which is positive and phase invariant; see part I [17, Fig.3]. Then the exponential curves through $g_{0}$ with direction $\left.c^{i} \mathcal{A}_{i} U\right|_{g_{0}}$ form "tangent spirals" (where we naturally embed $S E(2)$ into $\mathbb{R}^{2} \times[-\pi, \pi)$ ) to the orientation score $\mathcal{W}_{\psi} f: S E(2) \rightarrow \mathbb{R}^{+}$. In particular, the horizontal exponential curves are given by (3.22). See Figure 5 .

In this section we will provide fast algorithms for curvature estimation at each $g_{0} \in$ $S E(2)$ in the domain of $U$, by finding the exponential curve through $g_{0}$ that fits $U$ locally in an optimal way. 
REMARK 3.14. Sometimes we restrict ourselves to horizontal exponential curve fits; however, orientation scores $\mathcal{W}_{\psi} f$ and their absolute value $U=\left|\mathcal{W}_{\psi} f\right|$ in general do not satisfy $\partial_{\eta} U=0$. So from a strict point of view this restriction is not entirely appropriate. Nevertheless, the density $U=\left|\mathcal{W}_{\psi} f\right|$ is typically concentrated around horizontal curves. Recall, for example, Figure 1 in Part I of this article.

For the exact definition of such an optimally fitting (horizontal) tangent spiral we first need a few preliminaries. First we apply the left-invariant first fundamental form (3.13) on $T(S E(2)) \times T(S E(2))$. Then the norm of a left-invariant vector field $c^{i} \mathcal{A}_{i}$ equals

$$
\left|c^{i} \mathcal{A}_{i}\right|_{\beta}=\sqrt{\left(c^{i} \mathcal{A}_{i}, c^{i} \mathcal{A}_{i}\right)_{\beta}}=\sqrt{\left(c^{\theta}\right)^{2}+\left(\beta c^{\xi}\right)^{2}+\left(\beta c^{\eta}\right)^{2}}=:\|\mathbf{c}\|_{\beta},
$$

with $\mathbf{c}=\left(c^{1}, c^{2}, c^{3}\right) \in \mathbb{R}^{3}$. Here we stress that the norm $|\cdot|_{\beta}: \mathcal{L}(S E(2)) \rightarrow \mathbb{R}^{+}$ is defined on the space $\mathcal{L}(S E(2))$ of left-invariant vector fields on $S E(2)$, whereas the norm $\|\cdot\|_{\beta}: \mathbb{R}^{3} \rightarrow \mathbb{R}^{+}$is defined on $\mathbb{R}^{3}$. The gradient $\mathrm{d} U$ of $U: S E(2) \rightarrow \mathbb{R}^{+}$ is given by $\mathrm{d} U=\frac{\partial U}{\partial \theta} \mathrm{d} \theta+\frac{\partial U}{\partial \xi} \mathrm{d} \mathcal{A}^{2}+\frac{\partial U}{\partial \eta} \mathrm{d} \mathcal{A}^{3}$. The corresponding vector field equals $\mathcal{G}^{-1} \mathrm{dU}=\frac{\partial U}{\partial \theta} \partial_{\theta}+\beta^{-2} \frac{\partial U}{\partial \xi} \partial_{\xi}+\beta^{-2} \frac{\partial U}{\partial \eta} \partial_{\eta}$. Note that $\mathcal{G}^{-1} \mathrm{~d} \mathcal{A}^{k}=g^{k i} \mathcal{A}_{i}$, with $g^{i j} g_{j l}=\delta_{l}^{i}$.

The norm of a co-vector field (such as the gradient $\mathrm{d} U$ ) is given by

$$
\left|a_{i} \mathrm{~d} A^{i}\right|_{\beta}^{2}=g^{i j} a_{i} a_{j}=\left(a_{\theta}\right)^{2}+\beta^{-2}\left(a_{\xi}\right)^{2}+\beta^{-2}\left(a_{\eta}\right)^{2}=\|\mathbf{a}\|_{\beta^{-1}} \text { with } \mathbf{a}=\left(a^{1}, a^{2}, a^{3}\right) .
$$

If we differentiate a smooth function $U: S E(2) \rightarrow \mathbb{R}^{+}$along an exponential curve $\gamma(t)=g_{0} \exp \left(t\left(\sum c^{i} A_{i}\right)\right)$ passing $g_{0}$ we get, recall (3.24),

$$
\frac{d}{d t} U(\gamma(t))=\left.\sum_{i=1}^{3} c^{i} \mathcal{A}_{i} U\right|_{\gamma(t)}=c^{1} U_{\theta}(\gamma(t))+c^{2} U_{\xi}(\gamma(t))+c^{3} U_{\eta}(\gamma(t)) .
$$

After these preliminaries we return to our goal of finding the optimal tangent spiral at position $g_{0} \in S E(2)$ given $U: S E(2) \rightarrow \mathbb{R}^{+}$.

DEFINITION 3.15. Consider the solution of the following minimization problem:

$$
\mathbf{c}_{*}=\arg \min _{\left\{c^{i}\right\}_{i=1}^{3}}\left\{\left.\left|\frac{d}{d t} \mathrm{~d} U(\gamma(t))\right|_{t=0}\right|_{\beta} ^{2} \mid \gamma(t)=g_{0} \exp \left(t\left(\sum_{i=1}^{3} c^{i} A_{i}\right)\right) ;\|\mathbf{c}\|_{\beta}=1\right\} .
$$

Then we call the covariantly constant curve $t \mapsto g_{0} \exp \left(t \sum_{i=1}^{3} c_{*}^{i} A_{i}\right)$ the optimal tangent spiral at $g_{0} \in S E(2)$ given $U: S E(2) \rightarrow \mathbb{R}^{+}$.

By means of (3.40) and the chain rule, the energy in (3.41) can be rewritten as

$$
\begin{aligned}
& \left.\left|\frac{d}{d t}(\mathrm{~d} U)(\gamma(t))\right|_{t=0}\right|_{\beta} ^{2}=\left\|\nabla(\nabla U)^{T}(\gamma(0)) \cdot \gamma^{\prime}(0)\right\|_{\beta^{-1}}^{2} \\
& =\left\|\left.\left(\begin{array}{ccc}
\partial_{\theta}\left(\partial_{\theta} U\right) & \partial_{\xi}\left(\partial_{\theta} U\right) & \partial_{\eta}\left(\partial_{\theta} U\right) \\
\partial_{\theta}\left(\partial_{\xi} U\right) & \partial_{\xi}\left(\partial_{\xi} U\right) & \partial_{\eta}\left(\partial_{\xi} U\right) \\
\partial_{\theta}\left(\partial_{\eta} U\right) & \partial_{\xi}\left(\partial_{\eta} U\right) & \partial_{\eta}\left(\partial_{\eta} U\right)
\end{array}\right)\right|_{g_{0}}\left(\begin{array}{c}
c^{1} \\
c^{2} \\
c^{3}
\end{array}\right)\right\|_{\beta^{-1}}^{2}=:\left\|\left.H U\right|_{g_{0}} \mathbf{c}\right\|_{\beta^{-1}}^{2},
\end{aligned}
$$

where $\nabla U:=\left(\partial_{\theta} U, \partial_{\xi} U, \partial_{\eta} U\right)$ and where the noncovariant Hessian $H U$ does not coincide with the covariant Hessian form consisting of covariant derivatives of the Cartan connection, which we provided in Theorem 3.9. The covariant Hessian form equals

$$
\begin{aligned}
{\left[\nabla_{i} \nabla_{j} U\right] } & =\left[\nabla_{i} \mathcal{A}_{j} U\right]=\left[\mathcal{A}_{i} \mathcal{A}_{j} U+\Gamma_{i j}^{\lambda} \mathcal{A}_{\lambda} U\right] \\
& =\left(\begin{array}{lll}
\partial_{\theta}\left(\partial_{\theta} U\right) & \partial_{\theta}\left(\partial_{\xi} U\right) & \partial_{\theta}\left(\partial_{\eta} U\right) \\
\partial_{\xi}\left(\partial_{\theta} U\right) & \partial_{\xi}\left(\partial_{\xi} U\right) & \partial_{\xi}\left(\partial_{\eta} U\right) \\
\partial_{\eta}\left(\partial_{\theta} U\right) & \partial_{\eta}\left(\partial_{\xi} U\right) & \partial_{\eta}\left(\partial_{\eta} U\right)
\end{array}\right) .
\end{aligned}
$$


For example, in the 2 nd row and the 1 st column we have $\nabla_{1} \nabla_{2} U=\left(\partial_{\theta} \partial_{\xi}-c_{12}^{3} \partial_{\eta}\right) U=$ $\partial_{\xi}\left(\partial_{\theta} U\right)$. Note that the minimization problem (3.41) can now be rewritten as

$$
\arg \min _{\mathbf{c}}\left\{\left\|(H U)\left(g_{0}\right) \mathbf{c}\right\|_{\beta^{-1}}^{2} \mid\|\mathbf{c}\|_{\beta}=1\right\} .
$$

Set $M_{\beta}:=\operatorname{diag}\left\{1, \beta^{-1}, \beta^{-1}\right\} \in G L(3, \mathbb{R})$ and $H_{\beta} U:=M_{\beta} H U M_{\beta}$. Then by the EulerLagrange theory the gradient of $\|(H U) \mathbf{c}\|_{\beta^{-1}}^{2}=\left(\mathbf{c},(H U)^{T} M_{\beta}^{2}(H U) \mathbf{c}\right)_{1}$ at the optimum $\mathbf{c}_{*}$ is linearly dependent on the gradient of the side condition, which can be written $\left(1-\|\mathbf{c}\|_{\beta}^{2}\right)=1-\left(\mathbf{c}, M_{\beta}^{-2} \mathbf{c}\right)_{1}=0$, i.e.

$$
\left(H U\left(g_{0}\right)\right)^{T} M_{\beta}^{2}\left(H U\left(g_{0}\right)\right) \mathbf{c}_{*}=\lambda M_{\beta}^{-2} \mathbf{c}_{*} \Leftrightarrow\left(H_{\beta} U\right)^{T}\left(H_{\beta} U\right) \tilde{\mathbf{c}}=\lambda \tilde{\mathbf{c}},
$$

for some Lagrange multiplier $\lambda \in \mathbb{R}$, where $\tilde{\mathbf{c}}=M_{\beta}^{-1} \mathbf{c}_{*}$.

So we have shown that the minimization problem (3.41) requires eigensystem analysis of $\left(H_{\beta} U\right)^{T} H_{\beta} U$, which is the product of the Hessian $H_{\beta} U$ and the covariant Hessian given by (3.43), which equals $\left(H_{\beta} U\right)^{T}$. The covariant Hessian also appears in the EulerLagrange equation for the following minimization problem (for simplicity we set $\beta=1$ ):

$$
\arg \min _{c^{i}}\left\{\left|\frac{d^{2}}{d t^{2}} U(\gamma(t))\right| \mid \gamma(t)=g_{0} \exp \left(t\left(\sum_{i=1}^{3} c^{i} A_{i}\right)\right) ;\|\mathbf{c}\|_{\beta=1}=1\right\},
$$

which by means of a double application of (3.40) can be rewritten as

$$
\left|\frac{d^{2}}{d t^{2}} U(\gamma(t))\right|=\left|\frac{d}{d t} c^{i} \mathcal{A}_{i} U(\gamma(t))\right|=\left|c^{j} c^{i} \mathcal{A}_{j}\left(\mathcal{A}_{i} U\right)(\gamma(t))\right|=\left|\mathbf{c}^{T}\left(\left.\frac{1}{2}\left(H U+(H U)^{T}\right)\right|_{\gamma(t)}\right) \mathbf{c}\right|
$$

and as a result the Euler-Lagrange equations for the minimization problem (3.44) correspond to the eigensystem of $\frac{1}{2}\left(H U+(H U)^{T}\right): \nabla \nabla^{T} U \mathbf{c}=\frac{1}{2}\left(H U+(H U)^{T}\right) \mathbf{c}=\lambda \mathbf{c}$.

Experiments on images consisting of lines with ground truth curvatures show that the minimization problem (3.41) is preferable over (3.44) for spatial curvature estimation.

REMARK 3.16. On the commutative group $\mathbb{R}^{2}$ (i.e., the domain of images $f$ rather than the domain of the orientation scores $\mathcal{W}_{\psi} f$ ) we do not have the difference between the two Hessians above, since here the Hessian $H f=\left(\begin{array}{cc}f_{x x} & f_{x y} \\ f_{y x} & f_{y y}\end{array}\right)$ is square symmetric and thereby $H f=\frac{1}{2}\left(H f+(H f)^{T}\right)$ and $(H f)^{T}(H f)$ have the same eigenvectors with respective eigenvalues $\left\{\lambda_{n}\right\}$ and $\left\{\left(\lambda_{n}\right)^{2}\right\}$.

We suggest the following two methods for curvature estimation. In the first approach we do not restrict ourselves to horizontal exponential curves, whereas in the second approach we enforce horizontality and obtain a horizontal curvature estimate.

1. Compute the curvature of the projection $\mathbf{x}(s(t))=\mathbb{P}_{\mathbb{R}^{2}}\left(g_{0} \exp \left(t\left(\sum_{i=1}^{3} c_{*}^{i} A_{i}\right)\right)\right)$ of the optimal exponential curve in $S E(2)$ on the ground plane from an eigenvector $\mathbf{c}_{*}=\left(c_{*}^{\theta}, c_{*}^{\xi}, c_{*}^{\eta}\right)$. This eigenvector of $\left(\tilde{H}_{\beta}|U|\right)^{T}\left(\tilde{H}_{\beta}|U|\right)$, with $3 \times 3$ Hessian

$$
\tilde{H}_{\beta}|U|=\left(\begin{array}{ccc}
\beta^{2} \partial_{\theta} \partial_{\theta}|U| & \beta \partial_{\xi} \partial_{\theta}|U| & \beta \partial_{\eta} \partial_{\theta}|U| \\
\beta \partial_{\theta} \partial_{\xi}|U| & \partial_{\xi} \partial_{\xi}|U| & \partial_{\eta} \partial_{\xi}|U| \\
\beta \partial_{\theta} \partial_{\eta}|U| & \partial_{\xi} \partial_{\eta}|U| & \partial_{\eta} \partial_{\eta}|U|
\end{array}\right)
$$

is the one with smallest eigenvalue. The curvature estimation is now given by

$$
\kappa_{e s t}=\|\ddot{\mathbf{x}}(s)\| \operatorname{sign}\left(\ddot{\mathbf{x}}(s) \cdot \mathbf{e}_{\eta}\right)=\frac{c_{*}^{\theta} \operatorname{sign}\left(c_{*}^{\xi}\right)}{\sqrt{\left(c_{*}^{\xi}\right)^{2}+\left(c_{*}^{\eta}\right)^{2}}} .
$$


2. Compute the eigenvectors of $\left(\tilde{H}_{\beta}^{\text {hor }}|U|\right)^{T}\left(\tilde{H}_{\beta}^{\text {hor }}|U|\right)$ with horizontal Hessian

$$
\tilde{H}_{\beta}^{\text {hor }}|U|=\left(\begin{array}{cc}
\beta^{2} \partial_{\theta} \partial_{\theta}|U| & \beta \partial_{\xi} \partial_{\theta}|U| \\
\beta \partial_{\theta} \partial_{\xi}|U| & \partial_{\xi} \partial_{\xi}|U| \\
\beta \partial_{\theta} \partial_{\eta}|U| & \partial_{\xi} \partial_{\eta}|U|
\end{array}\right) .
$$

To this end we recall that the optimum $\mathbf{c}_{*}=\arg \min \left\{\left\|\tilde{H}_{\beta}^{\text {hor }}|U|\left(g_{0}\right) \mathbf{c}\right\|_{\beta^{-1}}^{2} \mid\|\mathbf{c}\|_{\beta}=1\right\}$ with $\mathbf{c}=\left(c^{\theta}, c^{\xi}\right)=c^{\theta} \mathbf{e}_{\theta}+c^{\xi} \mathbf{e}_{\xi}$ satisfies $2\left(\tilde{H}_{\beta}^{h o r}|U|\right)^{T} \tilde{H}_{\beta}^{h o r}|U| \tilde{\mathbf{c}}=2 \lambda \tilde{\mathbf{c}}, \mathbf{c}_{*}=M_{\beta} \tilde{\mathbf{c}}$ for some Lagrange multiplier $\lambda$. Then we compute the curvature of the projection $\mathbf{x}(s(t))=$ $\mathbb{P}_{\mathbb{R}^{2}}\left(g_{0} \exp \left(t\left(\sum c_{*}^{i} A_{i}\right)\right)\right)$ of the exponential curve in $S E(2)$ on the ground plane from the eigenvector $\mathbf{c}_{*}=\left(c_{*}^{\theta}, c_{*}^{\xi}\right)$ with smallest eigenvalue:

$$
\kappa_{\text {est }}^{\text {hor }}=\|\ddot{\mathbf{x}}(s)\| \operatorname{sign}\left(\ddot{\mathbf{x}}(s) \cdot \mathbf{e}_{\eta}\right)=\frac{c_{*}^{\theta}}{c_{*}^{\xi}} .
$$

For numerical experiments on the proposed curvature estimation (comparing the two methods above and the approach by van Ginkel [37]) on orientation scores of noisy example images, see [20], 19, [16].

4. Coherence-enhancing diffusion on orientation scores. In order to obtain adaptive diffusion on orientation scores we will use the following basic nonlinear leftinvariant evolution equation on $S E(2)$ as a starting point:

$$
\left\{\begin{array}{l}
\partial_{t} U(g, t)=\left(\begin{array}{lll}
\beta \partial_{\theta} & \partial_{\xi} & \partial_{\eta}
\end{array}\right)\left(\begin{array}{ccc}
\left(D_{11}(U)\right)(g, t) & 0 & 0 \\
0 & \left(D_{22}(U)\right)(g, t) & 0 \\
0 & 0 & \left(D_{33}(U)\right)(g, t)
\end{array}\right)\left(\begin{array}{c}
\beta \partial_{\theta} \\
\partial_{\xi} \\
\partial_{\eta}
\end{array}\right) U(g, t), \\
\text { for all } g \in S E(2), t>0, \\
U(g, t=0)=\mathcal{W}_{\psi}[f](g) \text { for all } g \in S E(2),
\end{array}\right.
$$

with $\beta>0$ (recall 3.8 ) and where the positive functions $D_{k k}: \mathbb{L}_{2}\left(S E(2) \times \mathbb{R}^{+}\right) \cap$ $C^{2}\left(S E(2) \times \mathbb{R}^{+}\right) \rightarrow C^{1}\left(S E(2) \times \mathbb{R}^{+}\right), k=1,2,3$ are given by

$$
(g, t) \mapsto\left(D_{k k}(U)\right)(g, t) \geq 0, \quad U \in \mathbb{L}_{2}\left(S E(2) \times \mathbb{R}^{+}\right) .
$$

These functions $D_{k k}, k=1,2,3$ should be chosen dependent on the local Hessian $H U(\cdot, t)$ of $U(\cdot, t)$ such that, at strong orientations, $D_{33}$ should be small so that we have anisotropic diffusion in the spatial plane along the preferred direction $\partial_{\xi}$, while, at weak orientations, $D_{33}$ and $D_{22}$ should be relatively large and isotropic $D_{22} \approx D_{33}$.

EXAMPLE. In the nonlinear diffusion system (4.1) we propose to set $D_{22}(U)(g, t)=1$, $D_{11}(U)(g, t)=D_{33}(U)(g, t)=e^{-\frac{(s(|U|)(g, t))^{2}}{c}}$, where $c>0$ is a standard nonlinear diffusion parameter and where the orientation confidence $s(U)(g, t)$ is given by

$$
s(U)(g, t)=\max \left\{-\left(\partial_{\eta}^{2}|U(g, t)|+\beta^{2} \partial_{\theta}^{2}|U(g, t)|\right), 0\right\} .
$$

With respect to the numerics of (4.1) (and later (4.4)), we implemented a forward finite difference scheme using central differences along the moving frame $\{\xi, \eta, \theta\}$, where we used second-order $B$-spline interpolation, [36, to get the equidistant samples on the $\{\xi, \eta, \theta\}$-grid from the given samples on the $\{x, y, \theta\}$-grid; see Figure 6. Our method is second-order accurate on $S E(2)$ and only first-order accurate in time. With this respect we note that a Crank-Nicholson scheme for time integration is second-order in time and 


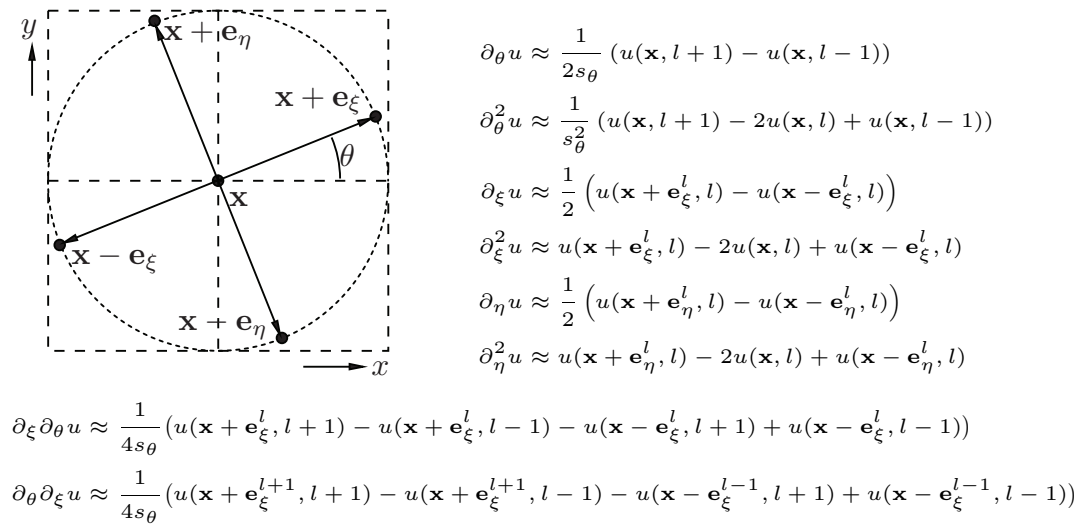

FIG. 6. Finite difference scheme of [4.1], where we use second-order $B$-spline interpolation, [36, for sampling on the grid of our moving frame $\left\{\mathbf{e}_{\theta}, \mathbf{e}_{\xi}=\cos \theta \mathbf{e}_{x}+\sin \theta \mathbf{e}_{y}, \mathbf{e}_{\eta}=-\sin \theta \mathbf{e}_{x}+\cos \theta \mathbf{e}_{y}\right\}$.

can improve computation time since one can take larger time steps. For comparison between coherence-enhancing diffusions on images and orientation scores, see Figure 7

4.1. Including adaptive curvatures in the diffusion scheme by gauge coordinates. In Subsection 3.4 we discussed two methods to obtain curvature estimates in orientation scores. This was done by finding the best exponential curve fit to the absolute value 9 $\left|\mathcal{W}_{\psi} f\right|: S E(2) \rightarrow \mathbb{R}^{+}$of the orientation score $\mathcal{W}_{\psi} f: S E(2) \rightarrow \mathbb{C}$. We distinguished between two approaches. In the first approach (3.46) we consider the best exponential curve fit to the absolute value of the orientation score, whereas in the second approach we consider the best horizontal exponential curve fit to the absolute value of an orientation score (3.48). Both approaches yield a curvature estimate, which in this paragraph we assume to be given. We shall write $\kappa:=\left(\kappa_{\text {est }}(|U|)\right)(g, t)$ for the curvature estimate of the score $U$ via its absolute value $|U|$ at location $g \in S E(2)$ at time $t>0$.

Now we can include curvature in our scheme (4.1) by replacing $\partial_{\xi}$ by $\partial_{\xi}+\kappa \partial_{\theta}$. To this end we recall from Theorem 3.9 that the exponential curve $s \mapsto g_{0} e^{\left.s\left(\partial_{\xi}+\kappa \partial_{\theta}\right)\right|_{e}}=$ $g_{0} e^{s\left(\partial_{x}+\kappa \partial_{\theta}\right)}$ yields a circular spiral (3.22) whose projection on $\mathbb{R}^{2}$ is a circle with radius $|\kappa|^{-1}$ if $\kappa$ is constant. Moreover, along horizontal curves we have (3.29); see Figure 8 ,

Here we note that $\left\{\partial_{\theta}, \partial_{\xi}+\kappa \partial_{\theta}, \partial_{\eta}\right\}$ are (in contrast to $\frac{1}{\beta}\left\{\beta \partial_{\theta}, \partial_{\xi}, \partial_{\eta}\right\}$ ) not orthonormal with respect to the $(\cdot, \cdot)_{\beta}$ inner product (3.39). Therefore we are going to introduce the gauge coordinates, aligned with the optimally fitting exponential curve

$$
s \mapsto g \exp \left(s \sum_{i=1}^{3} c_{*}^{i}(g, t) A_{i}\right), \quad \mathbf{c}_{*}(g, t)=\left(c_{*}^{\theta}(g, t), c_{*}^{\xi}(g, t), c_{*}^{\eta}(g, t)\right) \in \mathbb{R}^{3},
$$

\footnotetext{
${ }^{9}$ The absolute value $\left|\mathcal{W}_{\psi} f\right|$ is phase invariant; recall Fig. 3 in Part I of this article. This phase invariance is important for local feature estimation. Curvature estimation at the vicinity or border of a (thick) line should be similar to the estimate on top of the line.
} 


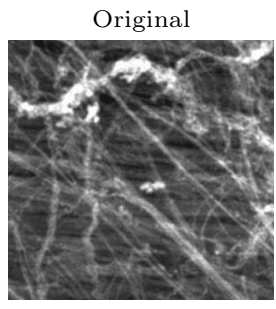

Original

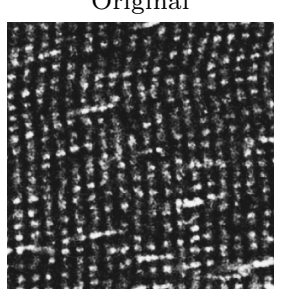

Original

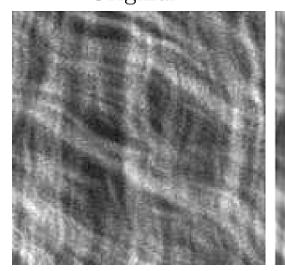

CED-OS $t=30$

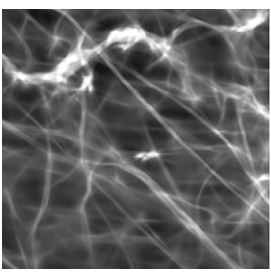

CED-OS $t=30$

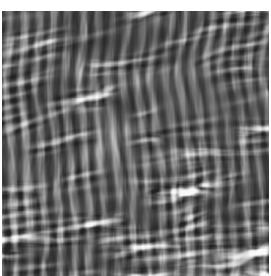

CED-OS $t=2$

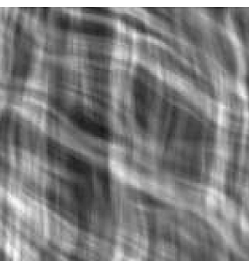

CED-OS $\quad \mathrm{t}=30$

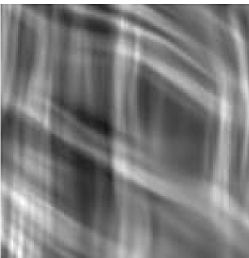

CED $t=30$

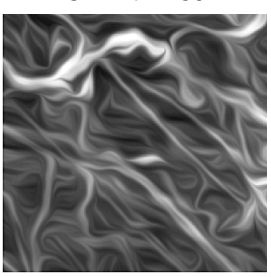

CED $t=30$

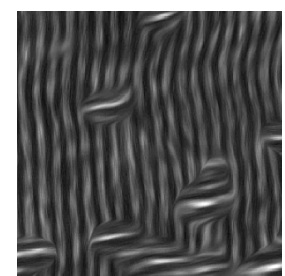

CED $\mathrm{t}=30$

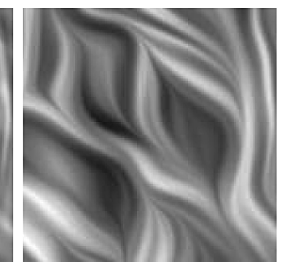

FIG. 7. Medical image applications. Top row: Result of coherenceenhancing diffusion on orientation scores (CED-OS), see (4.1) and (4.4), and standard coherence-enhancing diffusion (CED) directly on the image, see (2.5), of bone tissue. Middle row: Result of (CEDOS) and (CED) of 2-photon microscopy images of a muscle cell. Bottom row: Result of (CED-OS) and (CED) on medical images of collagen fibers of the heart. All these applications clearly show that coherence-enhancing diffusion on orientation scores (CED-OS) properly enhances crossing fibers whereas (CED) fails at crossings.

with $\left\|\mathbf{c}_{*}\right\|_{\beta}=\left(c_{*}^{\theta}\right)^{2}+\beta^{2}\left(c_{*}^{\xi}\right)^{2}+\beta^{2}\left(c_{*}^{\eta}\right)^{2}=1$, to the orientation score data $|U(\cdot, t)|$ at position $g \in S E(2)$ at time $t>0$. These gauge directions are (for $c_{*}^{\xi}>0$ ) given by

$$
\left\{\begin{array}{l}
\partial_{a}=\beta^{2} \sqrt{\left(c_{*}^{\xi}\right)^{2}+\left(c_{*}^{\eta}\right)^{2}} \partial_{\theta}-\frac{c_{*}^{\theta} c_{*}^{\xi}}{\sqrt{\left(c_{*}^{\xi}\right)^{2}+\left(c_{*}^{\eta}\right)^{2}}} \partial_{\xi}-\frac{c_{*}^{\theta} c_{*}^{\eta}}{\sqrt{\left(c_{*}^{\xi}\right)^{2}+\left(c_{*}^{\eta}\right)^{2}}} \partial_{\eta}, \\
\partial_{b}=\beta\left(c_{*}^{\xi} \partial_{\xi}+c_{*}^{\eta} \partial_{\eta}+c_{*}^{\theta} \partial_{\theta}\right) \\
\partial_{c}=\frac{-c_{*}^{\eta}}{\sqrt{\left(c_{*}^{\xi}\right)^{2}+\left(c_{*}^{\eta}\right)^{2}}} \partial_{\xi}+\frac{c_{*}^{\xi}}{\sqrt{\left(c_{*}^{\xi}\right)^{2}+\left(c_{*}^{\eta}\right)^{2}}} \partial_{\eta} .
\end{array}\right.
$$

Note that the gauge vector is along the best exponential curve-fit direction, i.e. $\partial_{b}=$ $\beta \mathbf{c}_{*}$ and $\operatorname{span}\left\{\partial_{a}, \partial_{c}\right\} \equiv\left(\mathbf{c}_{*}\right)^{\perp}$. For geometric understanding it helps to consider the gauge tangent vectors in the spherical coordinates $\left(d_{H}, \alpha\right)$ with respect to the basis of left-invariant vector fields $\left\{\partial_{\theta}, \partial_{\xi}, \partial_{\eta}\right\}$ so that it becomes obvious which rotation in $S O(3)$ (or rather which class of rotations in $S O(3) / S O(2) \equiv S^{2}$ if we do not distinguish between directions in the plane $\left.\left(\mathbf{c}_{*}\right)^{\perp}\right)$ is required to map the standard left-invariant basis $\left\{\partial_{\theta}, \partial_{\xi}, \partial_{\eta}\right\}$ into the gauge basis $\left\{\partial_{a}, \partial_{b}, \partial_{c}\right\}$; see Figure 9 . 
(a)
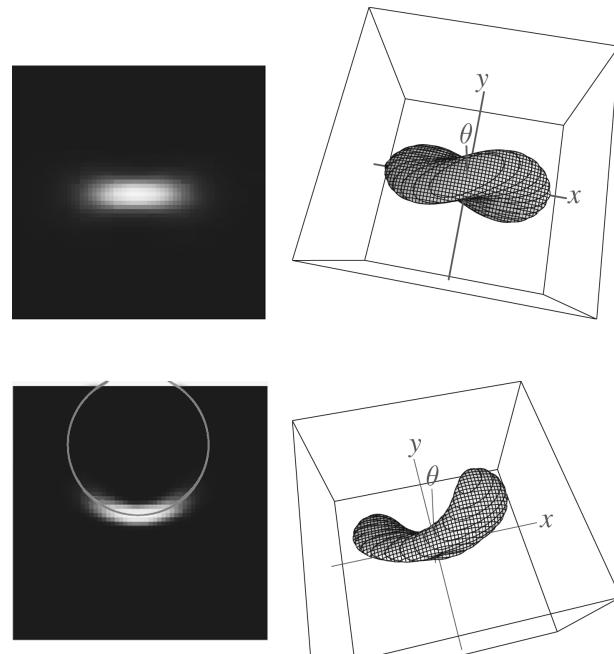

$D_{a a}=0$

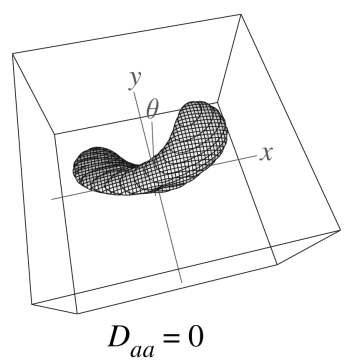

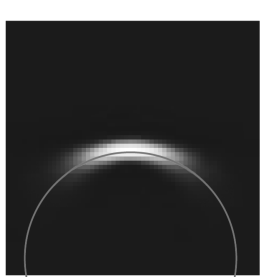

(c)

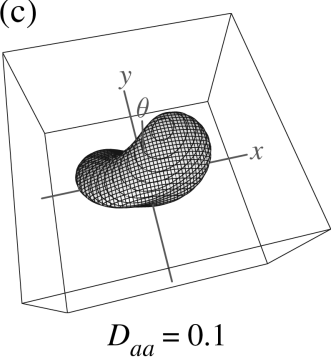

(b)
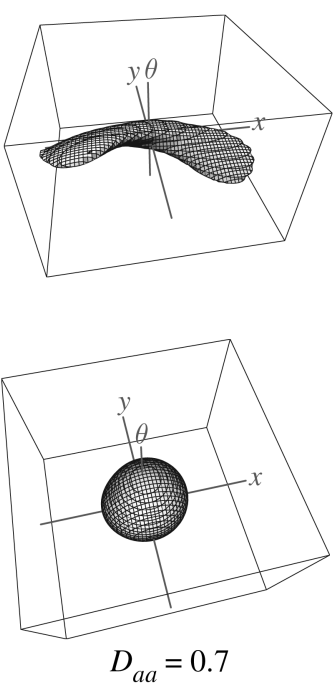

Fig. 8. Illustrations of heat kernels $K_{t}^{D}: S E(2) \rightarrow \mathbb{R}^{+}$on $S E(2)$ for different parameter values. (a): $D=\operatorname{diag}\left\{D_{11}, D_{22}, D_{33}\right\}$ in leftinvariant coordinate frame $\left\{\partial_{\theta}, \partial_{\xi}, \partial_{\eta}\right\}$, Left: heat kernel integrated over $\theta, D_{11}=D_{33}=0.003, \kappa=0, D_{22}=1$, and $\beta=1$. (b): shows the effect of nonzero $\kappa$. Parameters $\kappa=-0.04, D_{a a}=D_{c c}=0$, $D_{b b}=1$, and $\beta=1, d_{H}=0$ with respect to frame $\left\{\partial_{a}, \partial_{b}, \partial_{c}\right\}$, see (4.3). (c): Shows the effect of varying $D_{a a}=D_{c c}$. Parameters $\kappa=0.06, \beta=0.1$ and $D_{b b}=1$. As $D_{a a}$ increases from 0 to 1 , the resulting Green's function becomes more and more isotropic.

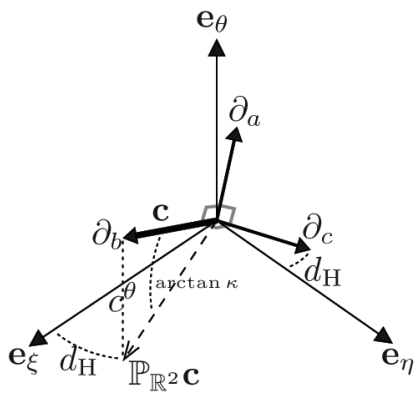

FIG. 9. Illustration of curvature $\kappa$ and deviation from horizontality $d_{H}$ and the gauge frame (4.3). For visualization reasons, the lengths of the vectors are arbitrary. The true lengths are given by $\left\|\mathbf{c}_{*}\right\|_{\beta}=$ $\left\|\mathbf{e}_{\theta}\right\|_{\beta}=\left\|\mathbf{e}_{\xi}\right\|_{\beta}=\left\|\mathbf{e}_{\eta}\right\|_{\beta}=1$ and $\left\|\partial_{a}\right\|_{\beta}=\left\|\partial_{b}\right\|_{\beta}=\left\|\partial_{c}\right\|_{\beta}=\beta$. Recall from part I of this article that $\mathbf{e}_{\theta} \leftrightarrow \partial_{\theta}, \mathbf{e}_{\xi} \leftrightarrow \partial_{\xi}, \mathbf{e}_{\eta} \leftrightarrow \partial_{\eta}$.

The gauge directions in spherical coordinates read

$$
\left\{\begin{array}{l}
\partial_{a}=-\cos \alpha \cos d_{H} \partial_{\xi}-\cos \alpha \sin d_{H} \partial_{\eta}+\beta \sin \alpha \partial_{\theta}, \\
\partial_{b}=\sin \alpha \cos d_{H} \partial_{\xi}+\sin \alpha \sin d_{H} \partial_{\eta}+\beta \cos \alpha \partial_{\theta}, \\
\partial_{c}=-\sin d_{H} \partial_{\xi}+\cos d_{H} \partial_{\eta}
\end{array}\right.
$$




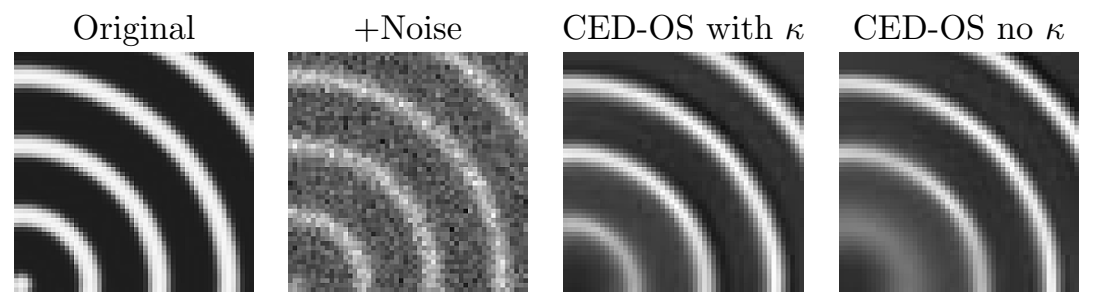

FIG. 10. Shows the effect of including curvature $\kappa=\left(\kappa_{\text {est }}(|U|)\right)(g, t)$ on a noisy test image $f$ in the final result of the CED-OS method, [20], [16], of $\Upsilon_{\psi} f=\mathcal{W}_{\psi}^{*} \Phi \mathcal{W}_{\psi} f$, where $\psi$ is the kernel illustrated in Fig. 3(e,f) of part I of this article and where operator $\Phi$ is the nonlinear diffusion (4.4) stopped at time $t=30$. At $t=30$ the effect is visible: the circle with highest curvature is blurred if no curvature is taken into account.
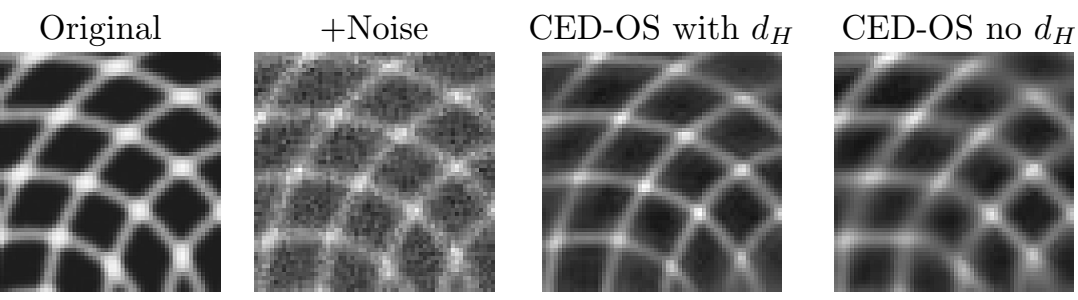

FIG. 11. Shows the effect of including "deviation from horizontality" $d_{H}(U)(g, t)=\arg \left(c_{*}^{\xi}(g, t)+i c_{*}^{\eta}(g, t)\right) \geq 0$ on a noisy test image $f$ in the final result $\Upsilon_{\psi} f=\mathcal{W}_{\psi}^{*} \Phi \mathcal{W}_{\psi} f$, where $\psi$ is the kernel illustrated in Fig. 3(e,f) of part I of this article and where the operator $\Phi$ is the nonlinear diffusion (4.4) stopped at time $t=24$ using only 4 equidistant samples on the circle. At $t=24$ the result without deviation from horizontality (using curvature estimation (3.48) ) clearly shows that the lines bias towards the sampled angles $0, \pi / 4, \pi / 2$ and $3 \pi / 4$. If we include deviation from horizontality (using curvature estimation (3.46) this problem does not occur, and even with only four samples on the torus we are able to handle these crossings correctly.

where the Euler angles read, where we recall (3.46),

$$
\alpha=\arccos \left(\operatorname{sign}\left(c_{*}^{\xi}\right) c_{*}^{\theta}\right)=\arccos \frac{\kappa}{\sqrt{\kappa^{2}+\beta^{2}}}, \quad d_{H}=\arg \left(c_{*}^{\xi}+i c_{*}^{\eta}\right) .
$$

Here the function $d_{H}$ which maps $U$ to $d_{H}(U)(g, t)=\arg \left(c_{*}^{\xi}(g, t)+i c_{*}^{\eta}(g, t)\right)$ represents the "deviation from horizontality", i.e. it indicates how much the tangent vector of the optimally fitting exponential curve (tangent spiral) points out of the horizontal plane, [20], 19]; see Figure 11].

REMARK 4.1. In some cases (for example if the image $f$ has many identical parallel curves) it is better to use the horizontal curvature estimates (3.48) rather than the curvature estimates (3.46), [20]. In these cases one must set $d_{H}=0$. 
The diagonal diffusion generator along the left-invariant gauge vector fields is

$$
\begin{aligned}
D_{a a}\left(\partial_{a}\right)^{2}+ & D_{b b}\left(\partial_{b}\right)^{2}+D_{c c}\left(\partial_{c}\right)^{2} \\
& =\left(\begin{array}{lll}
\beta \partial_{\theta} & \partial_{\xi} & \partial_{\eta}
\end{array}\right) M_{\alpha, d_{H}}^{T}\left(\begin{array}{ccc}
D_{a a} & 0 & 0 \\
0 & D_{b b} & 0 \\
0 & 0 & D_{c c}
\end{array}\right) M_{\alpha, d_{H}}\left(\begin{array}{l}
\beta \partial_{\theta} \\
\partial_{\xi} \\
\partial_{\eta}
\end{array}\right),
\end{aligned}
$$

where $M_{\alpha, d_{H}}=\left(\begin{array}{ccc}\sin \alpha & -\cos \alpha \cos d_{H} & -\cos \alpha \sin d_{H} \\ \cos \alpha & \cos d_{H} \sin \alpha & \sin \alpha \sin d_{H} \\ 0 & -\sin d_{H} & \cos d_{H}\end{array}\right)$ is the rotation matrix in $S O(3)$ that maps the left-invariant vector fields $\left\{\beta \partial_{\theta}, \partial_{\xi}, \partial_{\eta}\right\}$ onto the gauge frame $\left\{\partial_{a}, \partial_{b}, \partial_{c}\right\}$. This leads to the following nonlinear evolution equations on orientation scores:

$$
\left\{\begin{array}{l}
\partial_{t} U(g, t)=\left(\begin{array}{lll}
\beta \partial_{\theta} & \partial_{\xi} & \partial_{\eta}
\end{array}\right) M_{\alpha, d_{H}}^{T}\left(\begin{array}{ccc}
D_{a a} & 0 & 0 \\
0 & D_{b b} & 0 \\
0 & 0 & D_{c c}
\end{array}\right) M_{\alpha, d_{H}}\left(\begin{array}{c}
\beta \partial_{\theta} \\
\partial_{\xi} \\
\partial_{\eta}
\end{array}\right) U(g, t), t>0, \\
U(g, t=0)=\mathcal{W}_{\psi}[f](g) \text { for all } g \in S E(2),
\end{array}\right.
$$

where we again used short notation $D_{i i}=\left(D_{i i}(U)\right)(g, t)$, for $i=a, b, c$. Now again we set $D_{b b}=1$ and $\left(D_{a a}(U)\right)(g, t)=\left(D_{c c}(U)\right)(g, t)=e^{-\frac{(s(|U|)(g, t))^{2}}{c}}, c>0$, where orientation confidence $s(|U|)(g, t)$, recall (4.2), is now expressed in gauge coordinates:

$$
s(g, t)=\max \left\{-\Delta_{\mathbf{c}_{*}^{\perp}}|U(\cdot, t)|(g), 0\right\}=\max \left\{-\left(\left(\partial_{a}\right)^{2}|U(\cdot, t)|+\left(\partial_{c}\right)^{2}|U(\cdot, t)|\right)(g), 0\right\},
$$

and the conductivity matrix in (4.4) equals

$$
\frac{1}{\beta^{2}+\kappa^{2}}\left(\begin{array}{ccc}
\kappa^{2}+D_{a a} \beta^{2} & \kappa \beta\left(1-D_{a a}\right) \cos d_{H} & \kappa \beta\left(1-D_{a a}\right) \sin d_{H} \\
\kappa \beta\left(1-D_{a a}\right) \cos d_{H} & D_{a a}\left(\kappa^{2}+\beta^{2}\right)+\left(1-D_{a a}\right) \beta^{2} \cos ^{2} d_{H} & \cos d_{H} \sin d_{H} \beta^{2}\left(1-D_{a a}\right) \\
\kappa \beta\left(1-D_{a a}\right) \sin d_{H} & \cos d_{H} \sin d_{H} \beta^{2}\left(1-D_{a a}\right) & \beta^{2}+D_{a a} \kappa^{2}+\left(D_{a a}-1\right) \beta^{2} \cos ^{2} d_{H}
\end{array}\right) .
$$

See Figure 8 for an illustration of the special case that $D_{b b}$ is constant, $D_{a a}=D_{c c}=0$, $d_{H}=0$, which despite the strong degree of degeneracy still leads to a smooth and useful Green's function since the Hörmander condition, recall [17, Ch:5.3] (part I of this article) is satisfied. Furthermore, see Figure 10 and Figure 11 for illustrations of the practical relevance of, respectively, $\kappa$ and $d_{H}$ in the gauge coordinate frame (4.3).

Appendix A. Derivation of the geodesics in $P_{Y}$ by means of reduction of Pfaffian systems using Noether's Theorem. In this section we apply the BryantGriffiths approach [6] on the Marsden-Weinstein reduction for Hamiltonian systems [28] admitting a Lie group of symmetries on Euler-Lagrange equations associated to the functional $\int \sqrt{\kappa^{2}(s)+\epsilon} \mathrm{d} s$, to explicitly derive the solution curves $s \mapsto \gamma(s)$ in $S E(2)$. In [16, Ch:7.1] we derived the curvature of the minimizer of $\int \sqrt{\kappa^{2}(s)+\epsilon} \mathrm{d} s$ by solving an ODE for $\kappa$ that we derived from Euler-Lagrange minimization, similar to Mumford's approach to the elastica functional 29. Here we derive the same equation, in a more abstract way, avoiding extensive computations, by means of symplectic geometry. Moreover we will derive an important underlying conservation law and by the Marsden-Weinstein reduction we derive the curves themselves (rather than just their curvatures [16, Ch:7.1]). The formulae that we shall derive (A.6) for the geodesics are much simpler than the formulae for the corresponding elastica curves as they do not involve special functions. For small $\beta>0$ the geodesics are very close to the elastica curves and could be a nice practical 
alternative to the non-left-invariant (i.e. coordinate dependent) $B$-spline interpolations between two local orientations, [15, in vector graphics applications.

Although not discussed here, we stress that there is a fundamental relation between products of Green's functions discussed in Part I of this article, cf. [17, ch:4], and these curves: The unconditional Brownian bridge measure of the contour-enhancement process concentrates on the geodesics as the expected lifetime $E(T)=\frac{1}{\alpha} \rightarrow 0$; see [16, App.B].

Consider the manifold $Q=S E(2) \times \mathbb{R}^{+} \times \mathbb{R} \times \mathbb{R}$ with coordinates $\left(x, y, e^{i \theta}, \sigma, \kappa, t\right)$, where $\sigma=\left\|\mathbf{x}^{\prime}(t)\right\|$ so that $\mathrm{d} s=\sigma \mathrm{d} t$. On $Q$ we consider the Pfaffian equations

$$
\theta^{1}:=\mathrm{d} \mathcal{A}^{2}-\sigma \mathrm{d} t=0, \quad \theta^{2}:=\mathrm{d} \mathcal{A}^{3}=0, \quad \theta^{3}:=\mathrm{d} \theta-\kappa \sigma \mathrm{d} t=0 .
$$

Note that these Pfaffian equations uniquely determine the horizontal part $I(Q)$ of the dual tangent space $T^{*}(Q)$, where we recall that along horizontal curves we have $\frac{\mathrm{d} \theta}{\mathrm{ds}}=$ $\sigma^{-1} \frac{\mathrm{d} \theta}{\mathrm{dt}}=\kappa,\left\langle\mathrm{d} \mathcal{A}^{3}, \mathbf{x}^{\prime}(t)\right\rangle=0,\left\langle\mathrm{~d} \mathcal{A}^{2}, \mathbf{x}^{\prime}(t)\right\rangle=\sigma$.

We would like to minimize the energy $\int \sqrt{\kappa^{2}+\epsilon} \sigma \mathrm{d} t$ under the side conditions (A.1). Then the gradient of the energy should be linearly dependent on the gradient of the side condition, and therefore we set

$$
\psi=\sqrt{\kappa^{2}+\epsilon} \sigma \mathrm{d} t+\lambda_{1}(\mathrm{~d} \theta-\kappa \sigma \mathrm{dt})+\lambda_{2}\left(\mathrm{~d} \mathcal{A}^{2}-\sigma \mathrm{d} t\right)+\lambda_{3} \mathrm{~d} \mathcal{A}^{3},
$$

where $\lambda_{1}, \lambda_{2}, \lambda_{3}$ are Lagrange multipliers. Formally speaking, we consider the affine subbundle $Z=\left\{Z_{q} \mid q \in Q\right\} \equiv Q \times T(S E(2))^{*}$ of $T^{*}(Q)$ determined by

$$
\begin{aligned}
& Z_{q}=\left\{\left.\sqrt{\kappa^{2}+\epsilon} \sigma \mathrm{d} t\right|_{q} \in I_{q} \subset T_{q}^{*}(Q)\right\}, \\
& Z \equiv Q \times T(S E(2))^{*} \text { by the isomorphism }\left.(q, \boldsymbol{\lambda}) \leftrightarrow \sqrt{\kappa^{2}+\epsilon} \sigma \mathrm{d} t\right|_{q}+\left.\sum_{k=1}^{3} \lambda_{k} \theta^{k}\right|_{q} .
\end{aligned}
$$

Next we compute the exterior derivative of $\psi$ :

$$
\begin{aligned}
\mathrm{d} \psi & =\sqrt{\kappa^{2}+\epsilon} \mathrm{d} \sigma \wedge \mathrm{d} t+\frac{\kappa \sigma}{\sqrt{\kappa^{2}+\epsilon}} \mathrm{d} \kappa \wedge \mathrm{d} t+\lambda_{2} \mathrm{~d} \theta \wedge \mathrm{d} \mathcal{A}^{3}+\mathrm{d} \lambda_{2} \wedge \mathrm{d} \mathcal{A}^{2} \\
& -\mathrm{d} \lambda_{2} \wedge \sigma \mathrm{d} t-\lambda_{3} \mathrm{~d} \theta \wedge \mathrm{d} \mathcal{A}^{2}-\lambda_{2} \mathrm{~d} \sigma \wedge \mathrm{d} t+\mathrm{d} \lambda_{3} \wedge \mathrm{d} \mathcal{A}^{3}+\mathrm{d} \lambda_{1} \wedge \mathrm{d} \theta \\
& -\kappa \sigma \mathrm{d} \lambda_{1} \wedge \mathrm{d} t-\sigma \lambda_{1} \mathrm{~d} \kappa \wedge \mathrm{d} t-\kappa \lambda_{1} \mathrm{~d} \sigma \wedge \mathrm{d} t,
\end{aligned}
$$

where we used Cartan's structural equation (B.10): $\operatorname{dd} \mathcal{A}^{3}=-\mathrm{d} \theta \wedge \mathrm{d} \mathcal{A}^{2}, \operatorname{dd} \mathcal{A}^{2}=\mathrm{d} \theta \wedge \mathrm{d} \mathcal{A}^{3}$. Now by the results in [7, ch:1.2.2] the exterior derivative $\mathrm{d} \psi$ determines the characteristic curves (i.e. the geodesics) by means of

$$
\left.\gamma^{\prime}(t)\right\rfloor \mathrm{d} \psi_{\gamma(t)}=0, \quad \text { and } \gamma^{*} \mathrm{dt} \neq 0,
$$

where we use the notation $a\rfloor b:=b(a, \cdot)$. As a result the Pfaffian equations for decent parameterizations satisfying $\gamma^{*} \mathrm{dt} \neq 0$ are given by

$$
\left\{\begin{array}{l}
\left.\partial_{\lambda_{1}}\right\rfloor \mathrm{d} \psi=\mathrm{d} \theta-\kappa \sigma \mathrm{d} t=0 \\
\left.\partial_{\lambda_{2}}\right\rfloor \mathrm{d} \psi=\mathrm{d} \mathcal{A}^{2}-\sigma \mathrm{d} t=0 \\
\left.\partial_{\lambda_{3}}\right\rfloor \mathrm{d} \psi=\mathrm{d} \mathcal{A}^{3}=0 \\
\left.\partial_{\sigma}\right\rfloor \mathrm{d} \psi=\left(\sqrt{\kappa^{2}+\epsilon}-\lambda_{1} \kappa-\lambda_{2}\right) \mathrm{d} t=0 \\
\left.\partial_{\kappa}\right\rfloor \mathrm{d} \psi=\sigma\left(\kappa\left(\kappa^{2}+\epsilon\right)^{-1 / 2}-\lambda_{1}\right) \mathrm{d} t=0 \\
\left.-\partial_{\theta}\right\rfloor \mathrm{d} \psi=\mathrm{d} \lambda_{1}-\lambda_{2} \mathrm{~d} \mathcal{A}^{3}+\lambda_{3} \mathrm{~d} \mathcal{A}^{2}=0 \\
\left.-\partial_{\xi}\right\rfloor \mathrm{d} \psi=\mathrm{d} \lambda_{2}-\lambda_{3} \mathrm{~d} \theta=0 \\
\left.-\partial_{\eta}\right\rfloor \mathrm{d} \psi=\mathrm{d} \lambda_{3}+\lambda_{2} \mathrm{~d} \theta=0 .
\end{array}\right.
$$


The first three equations represent the horizontality restriction, the two equations in the middle represent the Euler-Lagrange optimization of the energy, and the last three equations provide the Lagrange multipliers

$$
\lambda_{1}=\frac{\kappa}{\sqrt{\kappa^{2}+\epsilon}}=z, \quad \lambda_{2}=\sqrt{\epsilon} \sqrt{1-z^{2}}, \quad \mathrm{~d} z+\lambda_{3} \sigma \mathrm{d} t=\mathrm{d} z+\lambda_{3} \mathrm{~d} s \Rightarrow \lambda_{3}=-\dot{z}
$$

As we will explain next, by Noether's theorem and an invariance group of symmetries of $\psi$ (in our case $S E(2)$ acting on $Z$ ) we will get important preservation laws along the characteristic curves.

Noether's theorem states that the momentum mapping $m: Z \rightarrow T_{e}(S E(2))^{*}$ given by

$$
\langle m(p), \xi\rangle=(\xi\rfloor \psi)(p), \quad p \in Z, \xi \in T_{e}(S E(2)),
$$

is constant along the characteristic curves.

Now the momentum mapping is invariant under the co-adjoint representation

$$
m\left(\eta_{g}(p)\right)=\left(\operatorname{Ad}_{g^{-1}}\right)^{*} m(p),
$$

where $\eta_{g}\left(p=\left(g^{\prime}, \kappa, \sigma, \lambda\right)\right)=\left(g g^{\prime}, \kappa, \sigma,\left(\operatorname{Ad}_{g^{-1}}\right)^{*} \lambda\right)$, so that $\eta_{g}^{*} \psi=\psi$ and $\left(\eta_{g}\right) \xi=\left(\operatorname{Ad}_{g}\right) \xi$, from which it indeed follows that

$$
\begin{aligned}
\left\langle m\left(\eta_{g} p\right), \xi\right\rangle & \left.=(\xi\rfloor \psi)\left(\eta_{g}(p)\right)=\left(\left(\eta_{g}\right)_{*} \xi\right\rfloor\left(\eta_{g}\right)^{*} \psi\right)(p) \\
& \left.\left.=\left(\left(\operatorname{Ad}_{g}\right) \xi\right\rfloor\left(\eta_{g}\right)^{*} \psi\right)(p)=(\xi\rfloor\left(\operatorname{Ad}_{g^{-1}}\right)_{*} \psi\right)(p) \\
& =\left\langle\left(\operatorname{Ad}_{g^{-1}}\right)_{*} m(p), \xi\right\rangle,
\end{aligned}
$$

for all $\xi \in T_{e}(S E(2))$. It can be verified that the co-adjoint orbits of $S E(2)$ are given by $\lambda_{2}^{2}+\lambda_{3}^{2}=c^{2} \epsilon \geq 0, \quad c>0$. Consequently, the geodesics are contained in the co-adjoint orbits, and we get the following preservation law that holds along the characteristic curves:

$$
(\dot{z}(s))^{2}+\epsilon-c^{2} \epsilon=\epsilon(z(s))^{2}, \quad s>0,
$$

where the normalized curvature $z(s)=\frac{\kappa(s)}{\sqrt{\kappa^{2}(s)+\epsilon}}$ satisfies $|z|<1$. Note that

$$
\ddot{z}=\epsilon z \Leftrightarrow \dot{z} \ddot{z}=\epsilon \dot{z} z \Leftrightarrow(\dot{z}(s))^{2}=\epsilon(z(s))^{2}+C, C \in \mathbb{R} .
$$

As observed by Bryant-Griffiths [6, pp. 543-544] (with slightly different conventions) the last three equations of (A.2) can be written as

$$
\mathrm{d} \hat{\lambda}=\hat{\lambda} g^{-1} \mathrm{~d} g \Leftrightarrow \mathrm{d}\left(\hat{\lambda} \cdot g^{-1}\right)=\mathrm{d}\left(\left(\widehat{\left.\mathrm{Ad}_{g^{-1}}\right)^{*}} \cdot \lambda\right)=0\right.
$$

where $\hat{\lambda}=\left(-\lambda_{3}, \lambda_{2}, \lambda_{1}\right)$ and where the matrix form of the Cartan connection equals

$$
g^{-1} \mathrm{~d} g=\left(\begin{array}{ccc}
\cos \theta & -\sin \theta & x \\
\sin \theta & \cos \theta & y \\
0 & 0 & 1
\end{array}\right) \quad \mathrm{d}\left(\begin{array}{ccc}
\cos \theta & -\sin \theta & x \\
\sin \theta & \cos \theta & y \\
0 & 0 & 1
\end{array}\right)=\left(\begin{array}{ccc}
0 & -\mathrm{d} \theta & \mathrm{d} \mathcal{A}^{2} \\
\mathrm{~d} \theta & 0 & \mathrm{~d} \mathcal{A}^{3} \\
0 & 0 & 0
\end{array}\right) \text {, }
$$

where both the Lie algebra and the Lie group are embedded in the group of invertible $3 \times 3$ matrices. Consequently, by Noether's theorem we have $\hat{\lambda}=\hat{\mu} \cdot g$, for some constant $\hat{\mu}=\left(-\mu_{3}, \mu_{2}, \mu_{1}\right)$, or more explicitly we have

$$
\left\{\begin{array}{l}
z=\mu_{1}-\mu_{3} x+\mu_{2} y \\
\dot{z}=-\mu_{3} \cos \theta+\mu_{2} \sin \theta \\
\sqrt{\epsilon\left(1-z^{2}\right)}=\mu_{3} \sin \theta+\mu_{2} \cos \theta, \quad \text { with } \mu_{2}^{2}+\mu_{3}^{2}=c^{2} \epsilon .
\end{array}\right.
$$


Next we choose

$$
h_{0}=\left(\begin{array}{ccc}
-\frac{\mu_{3}}{c \sqrt{\epsilon}} & -\frac{\mu_{2}}{c \sqrt{\epsilon}} & \frac{\mu_{1} \mu_{3}}{c^{2} \epsilon} \\
\frac{\mu_{2}}{c \sqrt{\epsilon}} & -\frac{\mu_{3}}{c \sqrt{\epsilon}} & -\frac{\mu_{1} \mu_{2}}{c^{2} \epsilon} \\
0 & 0 & 1
\end{array}\right)^{-1} \in S E(2)
$$

so that $\hat{\mu} \cdot h_{0}^{-1}=(\sqrt{\epsilon} c, 0,0)$ and use left-invariance $g=h_{0}^{-1} \tilde{g}, \tilde{g} \equiv\left(\tilde{x}, \tilde{y}, e^{i \tilde{\theta}}\right)$. Then we get $\hat{\lambda}=\hat{\mu} \cdot g=(c \sqrt{\epsilon}, 0,0) \cdot \tilde{g}$, i.e.

$$
\tilde{x}=\frac{z}{c \sqrt{\epsilon}}, \quad c \sqrt{\epsilon} \cos \tilde{\theta}=c \sqrt{\epsilon} \dot{\tilde{x}}=\dot{z}, \text { and }-\sqrt{\epsilon\left(1-z^{2}\right)}=-\sqrt{\epsilon} c \sin \tilde{\theta}=c \sqrt{\epsilon} \dot{\tilde{y}},
$$

and consequently we have

$$
\tilde{x}(s)=(\sqrt{\epsilon} c)^{-1} z(s), \quad \tilde{y}(s)=\tilde{y}(0)+\frac{1}{c} \int_{0}^{s} \sqrt{1-\left(z^{2}(\tau)\right)} \mathrm{d} \tau, \quad \tilde{\theta}(s)=\tilde{\theta}(0)+\int_{0}^{s} \kappa(\tau) \mathrm{d} \tau .
$$

So by solving (A.5) we have $z(s)=z_{0} \cosh (\sqrt{\epsilon} s)+\frac{z_{0}^{\prime}}{\sqrt{\epsilon}} \sinh (\sqrt{\epsilon} s)$ and we get the surprisingly simple solution $g(s)=(x(s), y(s), \theta(s))=h_{0}^{-1}(\tilde{x}(s), \tilde{y}(s), \tilde{\theta}(s))$, i.e.

$$
\left\{\begin{array} { l } 
{ x ( s ) = \frac { \mu _ { 1 } \mu _ { 3 } } { c ^ { 2 } \epsilon } - \frac { \mu _ { 3 } } { c \sqrt { \epsilon } } \tilde { x } ( s ) - \frac { \mu _ { 2 } } { c \sqrt { \epsilon } } \tilde { y } ( s ) } \\
{ y ( s ) = \frac { - \mu _ { 1 } \mu _ { 2 } } { c ^ { 2 } \epsilon } + \frac { \mu _ { 2 } } { c \sqrt { \epsilon } } \tilde { x } ( s ) - \frac { \mu _ { 3 } } { c \sqrt { \epsilon } } \tilde { y } ( s ) } \\
{ \theta ( s ) = \tilde { \theta } ( s ) + \operatorname { a r c c o s } ( - \frac { \mu _ { 3 } } { c \sqrt { \epsilon } } ) }
\end{array} \quad \text { ith } \left\{\begin{array}{l}
\tilde{x}(s)=\frac{z_{0}}{\sqrt{\epsilon \epsilon}} \cosh (\sqrt{\epsilon} s)+\frac{z_{0}^{\prime}}{c \epsilon} \sinh (\sqrt{\epsilon} s) \\
\tilde{y}(s)=\tilde{y} 0+\frac{1}{c} \int_{0}^{s} \sqrt{1-c^{2}(\tilde{x}(\tau))^{2} \epsilon} \mathrm{d} \tau \\
\tilde{\theta}(s)=\arccos \left(\frac{z_{0}}{c} \sinh (\sqrt{\epsilon} s)+\frac{z_{0}^{\prime}}{c \sqrt{\epsilon}} \cosh (\sqrt{\epsilon} s)\right)
\end{array}\right.\right.
$$

where $c=\sqrt{1+\frac{\left(z_{0}^{\prime}\right)^{2}}{\epsilon}-z_{0}^{2}}$. Now we have 6 unknown parameters $\mu_{1}, \mu_{3}, z_{0}, z_{0}^{\prime}, \tilde{y}(0), L$, to ensure the given boundary conditions

$$
\left\{\begin{array}{l}
g(0)=\left(x(0), y(0), e^{i \theta(0)}\right)=g_{0}:=\left(x_{0}, y_{0}, e^{i \theta_{0}}\right) \\
g(L)=\left(x(L), y(L), e^{i \theta(L)}\right)=g_{1}=\left(x_{1}, y_{1}, e^{i \theta_{1}}\right) .
\end{array}\right.
$$

By means of left-invariance we can always ensure (by multiplying from the left with $g_{1}^{-1}$ ) that $g_{1}=e$, so $\theta_{1}=0, x_{1}=0, y_{1}=0$. In this case straightforward computations yield

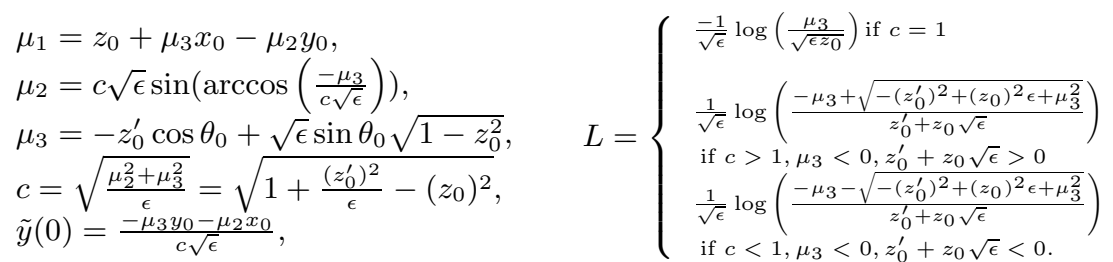

So all parameters are now expressed in the two unknowns $z_{0}$ and $z_{0}^{\prime}$, which are determined by the two remaining boundary conditions:

$$
\left\{\begin{array}{l}
\frac{\mu_{1} \mu_{3}}{c^{2} \epsilon}-\frac{\mu_{3}}{c \sqrt{\epsilon}} \tilde{x}(L)-\frac{\mu_{2}}{c \sqrt{\epsilon}} \tilde{y}(L)=x_{1}, \\
-\frac{\mu_{1} \mu_{2}}{c^{2} \epsilon}+\frac{\mu_{2}}{c \sqrt{\epsilon}} \tilde{x}(L)-\frac{\mu_{3}}{c \sqrt{\epsilon}} \tilde{y}(L)=y_{1} .
\end{array}\right.
$$

Now since $S E(2)$ is a symmetric space 24] all points can be connected by a geodesic and we may expect that there indeed exist $z_{0}$ and $z_{0}^{\prime}$ such that (A.8) holds. Consequently, the singularities (which cause extreme problems in our numerical shooting algorithm 16, Ch:7.1]), where $z\left(s_{\max }\right)=1$ occur always at $s_{\max } \geq L$ (and if $\mu_{3} \neq c \sqrt{\epsilon}$, then $s_{\max }>L$ ). Next we explicitly verify that $s_{\max } \geq L$ in two cases. 

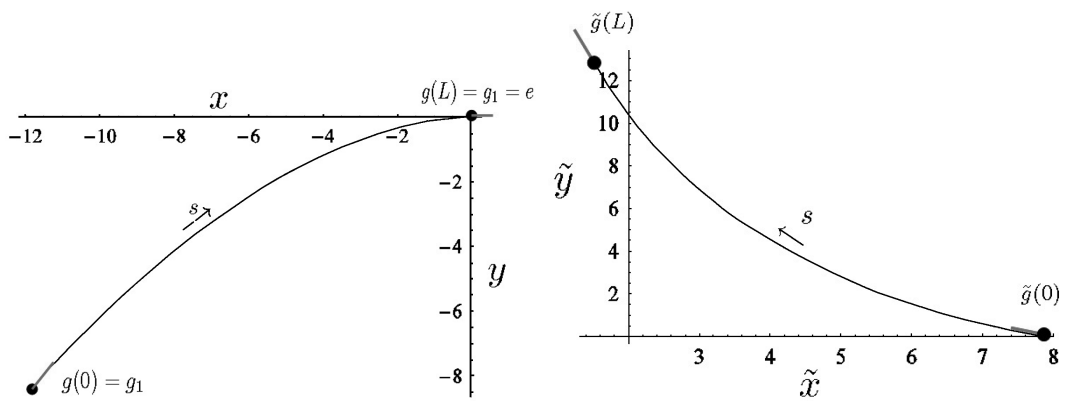

FIG. 12. Left figure: Illustration of a geodesic $s \mapsto g(s)$ computed by (A.6) and its affine relative $s \mapsto \tilde{g}(s)=h_{0}^{-1} g(s)$. Parameter settings $x_{0}=-11.868, y_{0}=-8.44337, \theta_{0}=51.95^{\circ}, x_{1}=y_{1}=\theta_{1}=0$, $L=15, \epsilon=0.0125, z_{0}=-0.1641, z_{0}^{\prime}=0.0183, c=1$.

In case $c>1, \mu_{3}<0$ and $z_{0}^{\prime}+\sqrt{\epsilon} z_{0}>0$ we have

$$
e^{\sqrt{\epsilon} L}=\frac{-\mu_{3}+\sqrt{-\left(z_{0}^{\prime}\right)^{2}+\left(z_{0}\right)^{2} \epsilon+\mu_{3}^{2}}}{z_{0}^{\prime}+z_{0} \sqrt{\epsilon}} \quad \text { and } e^{\sqrt{\epsilon} s_{\max }}=\frac{-\sqrt{\epsilon}+\sqrt{\left(z_{0}^{\prime}\right)^{2}-\left(z_{0}\right)^{2} \epsilon+\epsilon}}{z_{0}^{\prime}+z_{0} \sqrt{\epsilon}}=\frac{\sqrt{\epsilon}(1+c)}{z_{0}^{\prime}+z_{0} \sqrt{\epsilon}}
$$

and indeed $-\mu_{3}+\sqrt{-\left(z_{0}^{\prime}\right)^{2}+\left(z_{0}\right)^{2} \epsilon+\mu_{3}^{2}}<2 \sqrt{\epsilon}<(1+c) \sqrt{\epsilon}$, so $L<s_{\max }$.

In case $c<1, \mu_{3}>0$ and $z_{0}^{\prime}+\sqrt{\epsilon} z_{0}<0$ we have

$$
e^{\sqrt{\epsilon} L}=\frac{-\mu_{3}-\sqrt{-\left(z_{0}^{\prime}\right)^{2}+\left(z_{0}\right)^{2} \epsilon+\mu_{3}^{2}}}{z_{0}^{\prime}+z_{0} \sqrt{\epsilon}}
$$

and

$$
e^{\sqrt{\epsilon} s_{\max }}=\frac{-\sqrt{\epsilon}+\sqrt{\left(z_{0}^{\prime}\right)^{2}-\left(z_{0}\right)^{2} \epsilon+\epsilon}}{z_{0}^{\prime}+z_{0} \sqrt{\epsilon}}=\frac{\sqrt{\epsilon}(1+c)}{\left|z_{0}^{\prime}+z_{0} \sqrt{\epsilon}\right|}=\frac{\sqrt{\epsilon}(1+c)}{-\left(z_{0}^{\prime}+z_{0} \sqrt{\epsilon}\right)}
$$

and indeed we have $e^{\sqrt{\epsilon} s_{\max }} \geq e^{\sqrt{\epsilon} L}$, since

$$
\mu_{3}+\sqrt{-\left(z_{0}^{\prime}\right)^{2}+\left(z_{0}\right)^{2} \epsilon+\mu_{3}^{2}} \leq c \sqrt{\epsilon}+\sqrt{\epsilon\left(1-c^{2}\right)+c^{2} \epsilon}=\sqrt{\epsilon}(1+c) .
$$

Equality is obtained if $\mu_{3}=c \sqrt{\epsilon}$. See Figure 12,

Appendix B. Proof of Theorem 3.8. Again we set $\left\{A_{1}, A_{2}, A_{3}\right\}=\left\{\partial_{x}, \partial_{y}, \partial_{\theta}\right\}$ as a basis for the Lie algebra $T_{e}(S E(2))$ and $\left\{\mathcal{A}_{1}, \mathcal{A}_{2}, \mathcal{A}_{3}\right\}=\left\{\mathrm{d} \mathcal{R}\left(A_{1}\right), \mathrm{d} \mathcal{R}\left(A_{2}\right), \mathrm{d} \mathcal{R}\left(A_{3}\right)\right\}=$ $\left\{\partial_{\theta}, \partial_{\xi}, \partial_{\eta}\right\}$ as a basis for the space $\mathcal{L}(S E(2))$ of left-invariant vector fields with corresponding dual basis $\left\{\mathrm{d} \mathcal{A}^{1}, \mathrm{~d} \mathcal{A}^{2}, \mathrm{~d} \mathcal{A}^{3}\right\} \subset(\mathcal{L}(S E(2)))^{*}$ as given in Theorem 3.1

The Maurer-Cartan form $\omega:(S E(2), T(S E(2))) \rightarrow T_{e}(S E(2))$ is defined by

$$
\omega_{g}\left(Y_{g}\right)=\left(L_{g^{-1}}\right)_{*} Y_{g},
$$

where $\left(L_{g^{-1}}\right)_{*}$ denotes the push-forward of the inverse left multiplication $h \mapsto L_{g} h=$ $g^{-1} h$, i.e. $\omega_{g}\left(Y_{g}\right) \phi=Y_{g}\left(\phi \circ L_{g^{-1}}\right)$ for all $\phi: \Omega_{e} \rightarrow \mathbb{R}$ smooth and defined on some local open set $\Omega_{e}$ around the unity $e$. Now recall from Theorem 3.1 that the left-invariant vector fields are obtained by the derivative $\mathrm{d} \mathcal{R}$ of the right-regular representation $g \mapsto$ 
$\mathcal{R}_{g} \phi(h)=\phi(h g)$, i.e. $\mathcal{A}_{i}=\mathrm{d} \mathcal{R}\left(A_{i}\right)$. Now the Maurer-Cartan form does the reverse; it "connects" each tangent space $T_{g}(S E(2))$ to $T_{e}(S E(2))$. To this end we note that

$$
\lim _{h \downarrow 0} \frac{\phi\left(g \exp \left(h A_{i}\right)\right)-\phi(g)}{h}=:\left(\mathrm{d} \mathcal{R}\left(A_{i}\right)\right)_{g} \phi=\left(\mathcal{A}_{i}\right)_{g} \phi=\left(L_{g}\right)_{*} A_{i} \phi=A_{i}\left(\phi \circ L_{g}\right) \in \mathbb{R}
$$

for all $g \in S E(2)$ and all smooth $\phi: \Omega_{g} \rightarrow \mathbb{R}$. Therefore we have

$$
\left(L_{g}\right)_{*}\left(L_{g^{-1}}\right)_{*}=\left(L_{g g^{-1}}\right)_{*}=I \Leftrightarrow \forall_{i \in\{1,2,3\}}: \omega \circ \mathrm{d} \mathcal{R}\left(A_{i}\right)=\omega\left(\mathcal{A}_{i}\right)=A_{i} \Leftrightarrow \omega \circ \mathrm{d} \mathcal{R}=I .
$$

So by linearity and $\left\langle\mathrm{d} \mathcal{A}^{i}, \mathcal{A}_{j}\right\rangle=\delta_{i j}$ it is now clear that the coordinate-dependent definition of the Cartan-Maurer form (B.1) indeed yields (3.15) in explicit coordinates.

Now we show that the Maurer-Cartan form indeed forms a Cartan-Ehresmann connection form, recall Definition 3.6 on the principal fiber bundle and, recall Definition 3.5. $P=(S E(2), e, S E(2), \mathcal{L}(S E(2)))$. The first requirement in Definition 3.6 has been shown above, so let us verify the second requirement. Indeed a brief computation yields

$$
\omega_{g h}\left(\left(R_{h}\right)_{*} Y_{g}\right)=\left(L_{h^{-1}} \circ L_{g^{-1}}\right)_{*}\left(\left(R_{h}\right)_{*} Y_{g}\right)=\left(L_{h^{-1}}\right)_{*} \circ\left(L_{g^{-1}}\right)_{*} \circ\left(R_{h}\right)_{*} Y_{g}=\operatorname{Ad}\left(h^{-1}\right) \omega_{g} Y_{g} .
$$

Then we must show that equality (3.16) holds. This equality follows from the fact that left multiplication $L_{g}$ and right multiplication $R_{g}$ commute, since this implies that

$$
\left(R_{g^{-1}} L_{g}\right)_{*}=\left(L_{g} R_{g^{-1}}\right)_{*}=\left(L_{g}\right)_{*}\left(R_{g^{-1}}\right)_{*}=\left(L_{g}\right)_{*}\left(R_{g^{-1}} L_{g}\right)_{*}\left(L_{g^{-1}}\right)_{*},
$$

from which we indeed deduce that $\widetilde{\operatorname{Ad}}(g)=\mathrm{d} \mathcal{R} \circ \operatorname{Ad}(g) \circ \omega$. The adjoint representation Ad $: S E(2) \rightarrow G L\left(T_{e}(S E(2))\right)$ coincides with the derivative of the conjugation automor$\operatorname{phism} h \mapsto \operatorname{conj}(g)(h)=g h g^{-1}$ evaluated at $e$, i.e. $\operatorname{Ad}(g)=D_{e} \operatorname{conj}(g)=\left(R_{g^{-1}} L_{g}\right)_{*}$.

REMARK B.1. Here $G L\left(T_{e}(S E(2))\right)$ stands for all linear operators on the Lie algebra $T_{e}(S E(2))$. Note that each linear operator $\bar{Q} \in G L\left(T_{e}(S E(2))\right.$ on $\left.T_{e}(S E(2))\right)$ is 1-to-1 related to a bilinear form $Q$ on $\left(T_{e}(S E(2))\right)^{*} \times T_{e}(S E(2))$ by means of

$$
\begin{aligned}
& \langle B, \bar{Q} A\rangle=Q(B, A), \text { for all } B \in\left(T_{e}(S E(2))\right)^{*}, A \in T_{e}(S E(2)) \text { and } \\
& \bar{Q}=\sum_{i=1}^{3} Q\left(\mathrm{~d} A^{i}, \cdot\right) A_{i} .
\end{aligned}
$$

So a basis for $G L\left(T_{e}(S E(2))\right)$ is given by $\left\{\overline{\mathrm{d} A^{i} \otimes A_{j}} \mid i, j=1,2,3\right\}$. In this article, we omit the overline and again write $\mathrm{d} A^{i} \otimes A_{j}$ as it is clear from the context whether we mean the bilinear form or the linear mapping.

Recall Definition 3.7 of an associated vector bundle and set

$$
P=S E(2), M=e, G=S E(2), F=\mathcal{L}(S E(2)), \rho=\widetilde{\mathrm{Ad}}, \pi(g)=e, R_{g} u=u g,
$$

where $\mathcal{L}(S E(2))$ denotes the Lie algebra of left-invariant vector fields on $S E(2)$ and $\widetilde{\mathrm{Ad}}$ the adjoint representation of $S E(2)$ into $G L(\mathcal{L}(S E(2)))$ given by

$$
\widetilde{\operatorname{Ad}}(g) X=\left(R_{g^{-1}} L_{g}\right)_{*} X, \quad X \in \mathcal{L}(S E(2)), g \in S E(2) .
$$

A connection $\omega$ on a principal fiber bundle $P$ is 1-to-1 related to a connection $\tilde{\omega}$ on the vector bundle $P \times{ }_{\rho} F$ by means of

$$
\omega=\sum_{j} A_{j} \otimes \mathrm{d} x^{j} \leftrightarrow \tilde{\omega}=\sum_{j} \rho_{*}\left(A_{j}\right) \otimes \mathrm{d} x^{j},
$$


where $\left\{\mathrm{d} x^{j}\right\}$ are dual forms on $F$; for details on this common bijection, see [32. In our case we have $F=\mathcal{L}(S E(2))$ and dual forms $\left\{\mathrm{d} \mathcal{A}^{j}\right\}_{j=1,2,3}$. Note that we applied the convention in Remark B.1 So in our case (B.4) the push-forward $\rho_{*}$ of $\rho=\widetilde{\mathrm{Ad}}$ equals

$$
\begin{aligned}
(\widetilde{\mathrm{Ad}})_{*}\left(A_{j}\right) & =\left(\mathrm{d} \mathcal{R} \circ \mathrm{Ad}_{*}\right)\left(A_{j}\right)=(\mathrm{d} \mathcal{R} \circ \operatorname{ad} \circ \omega)\left(\mathcal{A}_{j}\right) \\
& =\widetilde{\operatorname{ad}}\left(\mathcal{A}_{j}\right)=\left[\cdot, \mathcal{A}_{j}\right]_{\mathcal{L}(S E(2))}=\sum_{i, k=1}^{3} c_{i j}^{k} \mathcal{A}_{k} \otimes \mathrm{d} \mathcal{A}^{i} .
\end{aligned}
$$

Thereby the connection form on the vector bundle $S E(2) \times \widetilde{\mathrm{Ad}} \mathcal{L}(S E(2))$ is given by

$$
\tilde{\omega}=\sum_{j=1}^{3} \widetilde{\operatorname{ad}}\left(\mathcal{A}_{j}\right) \otimes \mathrm{d} \mathcal{A}^{j}=\sum_{i, j, k=1}^{3} c_{i j}^{k} \mathcal{A}_{k} \otimes \mathrm{d} \mathcal{A}^{i} \otimes \mathrm{d} \mathcal{A}^{j},
$$

where $c_{i j}^{k}$ are the structure constants of the Lie group $S E(2)$, so $\widetilde{\operatorname{ad}}\left(\mathcal{A}_{j}\right)\left(\mathcal{A}_{i}\right)=\left[\mathcal{A}_{i}, \mathcal{A}_{j}\right]=$ $\sum_{i, j, k=1}^{3} c_{i j}^{k} \mathcal{A}_{k}$. The nonzero structure constants are $-c_{13}^{2}=c_{31}^{2}=c_{12}^{3}=-c_{21}^{3}=1$ and thereby (B.7) yields (3.17), where we also used $\mathrm{d} a \wedge \mathrm{d} b=\mathrm{d} a \otimes \mathrm{d} b-\mathrm{d} a \otimes \mathrm{d} b$.

Now from $\tilde{\omega}$ given by (B.7) one can construct the 9-connection 1-forms $\left\{\tilde{\omega}_{j}^{k}(\cdot)\right\}_{k, j=1}^{3}$ by (3.18) which together form a $3 \times 3$ matrix-valued 1 -form on the frame bundle [34, p.353, p.359], where the sections are moving frames [34, p.354].

Let $\left\{\mu_{k}\right\}_{k=1}^{3}$ denote the sections in $(S E(2), T(S E(2)))$ which coincide respectively with the left-invariant vector fields $\left\{\mathcal{A}_{k}\right\}_{k=1}^{3}$. Then the Cartan connection $D$ on (the vector bundle $S E(2) \times \widetilde{\mathrm{Ad}} \mathcal{L}(S E(2))$ isomorphic to) the tangent bundle $(S E(2), T(S E(2)))$ equals

$$
D:=\mathrm{d}+\bar{\omega} \text { with } \bar{\omega}\left(\mu_{k}\right)(\cdot):=\sum_{j=1}^{3} \tilde{\omega}_{k}^{j}(\cdot) \mu_{j},
$$

or more precisely, the covariant derivatives are given by

$$
\begin{aligned}
D_{\left.X\right|_{\gamma(t)}}(\mu(\gamma(t))) & :=(D \mu(\gamma(t)))\left(\left.X\right|_{\gamma(t)}\right) \\
& =\sum_{k=1}^{3} \dot{a}^{k}(t) \mu_{k}(\gamma(t))+\sum_{k=1}^{3} a^{k}(\gamma(t)) \sum_{j=1}^{3} \tilde{\omega}_{k}^{j}\left(\left.X\right|_{\gamma(t)}\right) \mu_{j}(\gamma(t)) \\
& =\sum_{k=1}^{3} \dot{a}^{k}(t) \mu_{k}(\gamma(t))+\sum_{i, j, k=1}^{3} \dot{\gamma}^{i}(t) a^{k}(\gamma(t)) \Gamma_{i k}^{j} \mu_{j}(\gamma(t)),
\end{aligned}
$$

with $\dot{a}^{k}(t)=\dot{\gamma}^{i}(t)\left(\left.\mathcal{A}_{i}\right|_{\gamma(t)} a^{k}\right)(\gamma(t))$, for all curves $\gamma: \mathbb{R} \rightarrow S E(2)$ and tangent vectors $\left.X\right|_{\gamma(t)}=\left.\sum_{i=1}^{3} \dot{\gamma}^{i}(t) \mathcal{A}_{i}\right|_{\gamma(t)} \in T_{\gamma(t)}(S E(2))$ and all sections

$$
\mu(\gamma(t))=\sum_{k=1}^{3} a^{k}(\gamma(t)) \mu_{k}(\gamma(t))
$$

and where the Christoffel symbols $\Gamma_{i j}^{k}$, [24, p.108], are constant: $\Gamma_{i j}^{k}=$ $\tilde{\omega}_{j}^{k}\left(\mathcal{A}_{i}\right)=-\tilde{\omega}\left(\mathrm{d} \mathcal{A}^{k}, \mathcal{A}_{i}, \mathcal{A}_{j}\right)=-c_{i j}^{k}=c_{j i}^{k}$. Now finally, the curvature 2-forms $\Omega_{j}^{i}$ for the moving frame $\left\{\mathcal{A}_{1}, \mathcal{A}_{2}, \mathcal{A}_{3}\right\}$ are $\Omega_{j}^{i}=\mathrm{d} \tilde{\omega}_{j}^{i}+\sum_{k=1}^{3} \tilde{\omega}_{k}^{i} \wedge \tilde{\omega}_{j}^{k}$, [34, p.317]. These curvature 2-forms are bijectively related, [34, p.322] to the Riemann-curvature tensor on the tangent bundle $(S E(2), T(S E(2)))$ :

$$
R_{j k l}^{i}=\Omega_{j}^{i}\left(\mathcal{A}_{k}, \mathcal{A}_{l}\right) \text { and } \Omega_{j}^{i}=\frac{1}{2} \sum_{k, l=1}^{3} R_{j k l}^{i} \mathrm{~d} \mathcal{A}^{k} \wedge \mathrm{d} \mathcal{A}^{l} .
$$


Now by means of Maurer-Cartan's structural formula, [1], recall (3.31),

$$
\mathrm{d}\left(\mathrm{d} \mathcal{A}^{k}\right)=-\frac{1}{2} \sum_{i, j, k=1}^{3} c_{i j}^{k} \mathrm{~d} \mathcal{A}^{\mathrm{i}} \wedge \mathrm{d} \mathcal{A}^{j},
$$

we find by straightforward computation (using Einstein's summation convention)

$$
\begin{aligned}
\Omega_{j}^{i} & =c_{j \lambda}^{i} \mathrm{~d}\left(\mathrm{~d} \mathcal{A}^{\lambda}\right)+c_{\lambda \mathrm{k}}^{\mathrm{i}} \mathrm{c}_{\mathrm{jl}}^{\lambda} \mathrm{d} \mathcal{A}^{\mathrm{k}} \wedge \mathrm{d} \mathcal{A}^{1}=\frac{1}{2}\left(c_{\mathrm{k} \lambda}^{\mathrm{i}} c_{1 j}^{\lambda}+\left(c_{j}^{\mathrm{i}} c_{\mathrm{kl}}^{\lambda}-c_{\mathrm{k} \lambda}^{\mathrm{i}} c_{j 1}^{\lambda}\right)\right) \mathrm{d} \mathcal{A}^{\mathrm{k}} \wedge \mathrm{d} \mathcal{A}^{1} \\
& =\frac{1}{2}\left(c_{k \lambda}^{i} c_{l j}^{\lambda}-c_{l \lambda}^{i} c_{k j}^{\lambda}\right) \mathrm{d} \mathcal{A}^{k} \wedge \mathrm{d} \mathcal{A}^{l}=\frac{1}{2} c_{\lambda j}^{i} c_{k l}^{\lambda} \mathrm{d} \mathcal{A}^{k} \wedge \mathrm{d} \mathcal{A}^{l}=\frac{1}{2} R_{i, k l}^{j} \mathrm{~d} \mathcal{A}^{k} \wedge \mathrm{d} \mathcal{A}^{l},
\end{aligned}
$$

where we used the Jacobi identity $[X,[Y, Z]]+[Y,[Z, X]]+[Z,[X, Y]]=0$ in the third and fourth equality. For example for $Y=\mathcal{A}_{l}, Z=\mathcal{A}_{j}, X=\mathcal{A}_{k}$ we find $c_{k \lambda}^{i} c_{l j}^{\lambda}+c_{l \lambda}^{i} c_{j k}^{\lambda}+c_{j \lambda}^{i} c_{k l}^{\lambda}$. Now from (B.11) we deduce $R_{j, k l}^{i}=\frac{1}{2} c_{\lambda j}^{i} c_{k l}^{\lambda}$, from which the final result (3.20) follows.

Acknowledgements. The authors are grateful to the reviewer for his good suggestions on the organization and formulation of this paper. Moreover, the authors gratefully acknowledge his thoughtful comments on the contents of earlier drafts. They also express their thanks to David Mumford, Brown University, USA, for his encouragement in the course of the work leading up to the paper.

\section{REFERENCES}

[1] M. A. Akivis and B.A. Rosenfeld. Élie Cartan (1869-1951), Translations of Mathematical Monographs. volume 123. American Mathematical Society, Providence, 1993. MR1222194 (94f:01029)

[2] V. Arsigny, X. Pennec and N. Ayache. Bi-invariant Means in Lie Groups. Application to Leftinvariant Polyaffine Transformations. Technical report, no. 5885, INRIA, France, April 2006.

[3] V. Arsigny, P. Fillard, X. Pennec and N. Ayache. Geometric Means in a Novel Vector Space Structure on Symmetric Positive-Definite Matrices. SIAM Journal on Matrix Analysis and Applications, 29(1):328-347, 2006. MR2288028 (2008a:47030)

[4] T. Aubin. A Course in Differential Geometry, Graduate Studies in Mathematics, vol.27, American Mathematical Society, 2001. MR:1799532(2001m:53001)

[5] J. August and S.W. Zucker. The curve indicator random field and Markov processes. IEEE-PAMI, Pattern Recognition and Machine Intelligence, 25, 2003. Number 4.

[6] R. Bryant and P. Griffiths. Reduction for constrained variational problems and $(1 / 2) \int \kappa^{2}$ ds. American Journal of Mathematics, 108(3):525-570, June 1986. MR.844630 (88a:58044)

[7] R. Bryant, P. Griffiths and D. Grossman. Exterior Differential Systems and Euler-Lagrange Partial Differential Equations. Chicago Lectures in Mathematics, 2003. MR1985469 (2004g:58001)

[8] G.S. Chirikjian and A.B. Kyatkin. Engineering applications of noncommutative harmonic analysis with emphasis on rotation and motion groups. CRC Press, Boca Raton, FL, USA, 2001. MR1885369 (2003g:42001)

[9] G. Citti and A. Sarti. A cortical based model of perceptional completion in the roto-translation space. Journal of Mathematical Imaging and Vision, 24 (3):307-326, 2006. MR.2235475 (2007b:92016)

[10] G.-H. Cottet and L. Germain. Image processing through reaction combined with nonlinear diffusion. Mathematics of Computation, 61:659-667, 1993. MR.1195422 (94a:65066)

[11] R. Duits. Perceptual Organization in Image Analysis. Ph.D. thesis, Eindhoven University of Technology, Department of Biomedical Engineering, The Netherlands, 2005. A digital version is available on the web URL: http:// www.bmi2.bmt.tue.nl/ImageAnalysis/People/RDuits/THESISRDUITS.pdf.

[12] R. Duits, M. Felsberg, G. Granlund, and B.M. ter Haar Romeny. Image analysis and reconstruction using a wavelet transform constructed from a reducible representation of the Euclidean motion group. International Journal of Computer Vision. Volume 72, issue 1, p.79-102, April 2007.

[13] R. Duits, M. Duits, M. van Almsick and B.M. ter Haar Romeny. Invertible Orientation Scores as an Application of Generalized Wavelet Theory. Image Processing, Analysis, Recognition, and Understanding. Volume 17, No. 1: pp. 42-75, 2007. 
[14] R. Duits, M. van Almsick, M. Duits, E. Franken, and L.M.J. Florack. Image processing via shifttwist invariant operations on orientation bundle functions. In Niemann Zhuralev et al. Geppener, Gurevich, editor, 7th International Conference on Pattern Recognition and Image Analysis: New Information Technologies, pages 193-196, St. Petersburg, October 2004.

[15] R. Duits and M. van Almsick. The Explicit Solutions of Linear Left-Invariant Second Order Stochastic Evolution Equations on the 2D Euclidean Motion Group. Quarterly of Applied Mathematics, Volume 66, no. 1, pp. 27-67, April 2008. MR2396651

[16] R. Duits and E.M. Franken. Left-invariant Stochastic Evolution Equations on $S E(2)$ and its Applications to Contour Enhancement and Contour Completion via Invertible Orientation Scores. arXiv: 0711.0951v4, see http://arxiv.org/abs/0711.0951. Also available as CASA report no. 35, 2007, Eindhoven University of Technology, http://www.win.tue.nl/casa/research/casareports/2007.html

$[17]$ R. Duits and E.M. Franken. Left-invariant Parabolic Evolutions on $S E(2)$ and Contour Enhancement via Invertible Orientation Scores, Part I: Linear Left-invariant Diffusion Equations on SE(2). Quarterly of Applied Mathematics, this volume.

[18] E. Franken, R. Duits and B.M. ter Haar Romeny. Nonlinear Diffusion on the Euclidean Motion Group. Proc. of the first international conference on Scale Space and Variational Methods in Computer Vision, 461-472, 2007.

[19] E.M. Franken, R. Duits and B.M. ter Haar Romeny. Curvature estimation for Enhancement of Crossing Curves. Digital Proc. of the 8th IEEE Computer Society Workshop on Mathematical Methods in Biomedical Image Analysis, held in conjuction with the IEEE Int. Conf. on Computer Vision (Rio de Janeiro). Omnipress, October 2007. Awarded the MMBIA 2007 best paper award.

[20] E.M. Franken and R. Duits. Crossing-Preserving Coherence-Enhancing Diffusion on Invertible Orientation Scores. Accepted, to appear in International Journal of Computer Vision, 2009.

[21] B. Gaveau. Principe de moindre action, propagation de la chaleur et estimées sous elliptiques sur certains groupes nilpotents. Acta Mathematica, 139:96-153, 1977. MR0461589 (57:1574)

[22] W. Hebisch. Estimates on the semigroups generated by left-invariant operators on Lie groups. Journal fuer die reine und angewandte Mathematik, 423:1-45, 1992. MR.1142482 (93d:22008)

[23] L. Hörmander. Hypoelliptic second order differential equations. Acta Mathematica, 119:147-171, 1967. MR0222474 (36:5526)

[24] J. Jost. Riemannian Geometry and Geometric Analysis, Springer, Heidelberg-New York, ed. 4, 2005. MR2165400 (2006c:53002)

[25] S. N. Kalitzin, B. M. ter Haar Romeny, and M. A. Viergever. Invertible apertured orientation filters in image analysis. Int. Journal of Computer Vision, 31(2/3):145-158, April 1999.

[26] S. Kobayashi and K. Nomizu. Foundations of Differential Geometry, Vol.1. Wiley Classics Library, New York, 1963. MR0152974 (27:2945)

[27] G.W. Mackey. Imprimitivity for representations of locally compact groups Proc. Nat. Acad. Sci. 35: p.537-545, 1949. MR0031489 (11:158b)

[28] J. Marsden and A. Weinstein. Reduction of symplectic manifolds with symmetry. Rep. Math. Phys., 5:121-130, 1974. MR0402819(53:6633)

[29] D. Mumford. Elastica and computer vision. Algebraic Geometry and Its Applications. SpringerVerlag, pages 491-506, 1994. MR1272050 (95a:92026)

[30] M. Nitzberg and T. Shiota. Nonlinear image filtering with edge and corner enhancement. IEEE Transactions on Pattern Analysis and Machine Intelligence, 14:826-833, 1992.

[31] P. Perona and J. Malik. Scale-space and edge detection using anisotropic diffusion. IEEE Transactions on Pattern Analysis and Machine Intelligence, (7).

[32] H.G.J. Pijls. The Yang-Mills equations. In proc. seminar 1981-1982. Mathematical structures in field theories, ed. E.M. de Jager and H.G.J. Pijls, pp.119-182, 1984. MR802640

[33] N.A. Sochen. Stochastic Processes in Vision I: From Langevin to Beltrami. Center for Communication and Information Technologies, Department of Electrical Engineering, Technion, Israël Institute of Technology, Haifa 32000, Israël, June 1999, Tech. rep. CC PUB \#285.

[34] M. Spivak. Differential geometry, Vol. II, Publish or Perish Inc., 1975.

[35] S. Sternberg. Lectures on Differential Geometry. Prentice Hall Math. Series, Prentice Hall Inc., 1964.

[36] M. Unser. A perfect fit for signal and image processing. IEEE signal processing magazine, pages 27-37, November 1999. 
[37] M. van Ginkel. Image Analysis using Orientation Space based on Steerable Filters, Ph.D. thesis, Delft University of Technology, Department of Biomedical Engineering, Eindhoven the Netherlands, October 2002.

[38] J. A. Weickert. Anisotropic Diffusion in Image Processing. ECMI Series. Teubner, Stuttgart, January 1998. MR.1666943(2000a:94003)

[39] J. A. Weickert. Coherence-enhancing diffusion filtering. International Journal of Computer Vision, 31(2/3):111-127, April 1999. 